\title{
OVERCOMING THE CHALLENGES OF USING UNMANNED AIRCRAFT FOR BRIDGE INSPECTIONS
}

\author{
A THESIS IN \\ Mechanical Engineering \\ Presented to the Faculty of the University \\ of Missouri-Kansas City in partial fulfillment of \\ the requirements for the degree

\section{MASTER OF SCIENCE}

by
IGNACIO HERNANDEZ \\ B. S., University of Missouri-Kansas City, 2015
}

Kansas City, Missouri

2016 
(c) 2016

IGNACIO HERNANDEZ

ALL RIGHTS RESERVED 


\title{
OVERCOMING THE CHALLENGES OF USING UNMANNED AIRCRAFT FOR BRIDGE INSPECTIONS
}

\author{
Ignacio Hernandez, Candidate for the Master of Science Degree \\ University of Missouri-Kansas City, 2016
}

\begin{abstract}
Applications for small unmanned aerial vehicles have exploded over the past decade. Small size, maneuverability, low cost, and versatility enable utilization for applications not readily accessible by traditional manned aircraft, such as bridge inspections. However, the challenges associated with conducting inspections in a safe, cost efficient manner currently exceed the benefits of using an unmanned aircraft for inspection. The development of a multirotor-based inspection aircraft capable of maintaining flight for over eight hours is presented. Flight results from a bridge inspection conducted in collaboration with the Missouri Department of Transportation (MoDOT) and Shafer, Kline, \& Warren (SKW) bridge inspection crews are also discussed. Feedback and recommendations for inspection platform improvement from MoDOT and SKW inspectors was collected. Inspectors and flight crew identified challenges with camera exposure, dual pilotinspector remote control, and aircraft stability. As a result, a new inspection platform was
\end{abstract}


developed allowing for wireless dual remote control and manual control of camera exposure. Finally, and most importantly, a new metric for quantifying UAS handling and pilot workload in GPS denied environments is introduced through rigorous flight testing and the use of a modified Cooper-Harper rating scale. Inspection of the bridge substructure prevented GPS lock and increased pilot workload. To mimic the effects of flying around a bridge flight testing is conducted at the UMKC parking garage where GPS lock is denied. The aircraft and inspection solutions discussed herein have demonstrated success for acquiring meaningful inspection data while conducting safe flight operations. 


\section{APPROVAL PAGE}

The faculty listed below, appointed by the Dean of the School of Computing and Engineering, have examined a thesis titled "OVERCOMING THE CHALLENGES OF USING UNMANNED AIRCRAFT FOR BRIDGE INSPECTIONS,” presented by Ignacio Hernandez, candidate for the Master of Science degree, and hereby certify that in their opinion it is worthy of acceptance.

\section{$\underline{\text { Supervisory Committee }}$}

Travis Fields, Ph.D., Committee Chair

Department of Civil \& Mechanical Engineering

John Kevern, Ph.D.

Department of Civil \& Mechanical Engineering

Gregory King, Ph.D.

Department of Civil \& Mechanical Engineering 


\section{CONTENTS}

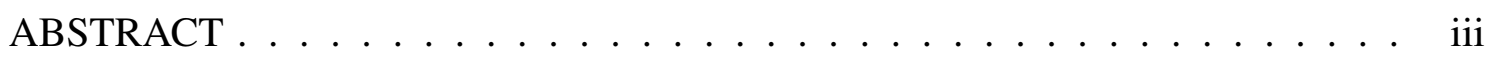

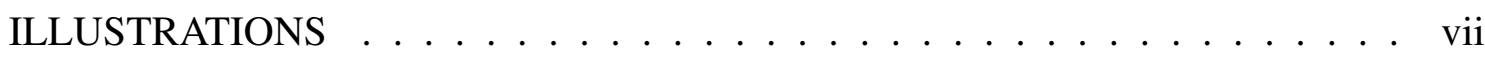

TABLES ........................

LIST OF ABBREVIATIONS ..................... xi

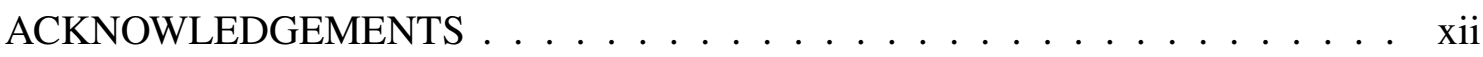

Chapter

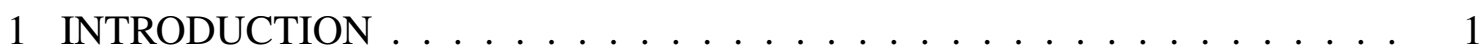

2 BACKGROUND \& LITERATURE REVIEW . . . . . . . . . . . . . . . 7

3 FIRST GENERATION PROTOTYPE . . . . . . . . . . . . . . . . 20

4 SECOND GENERATION PROTOTYPE . . . . . . . . . . . . . . . . . . 30

5 THIRD GENERATION PROTOTYPE . . . . . . . . . . . . . . . . . 41

6 FOURTH GENERATION PROTOTYPE . . . . . . . . . . . . . . . . . . 51

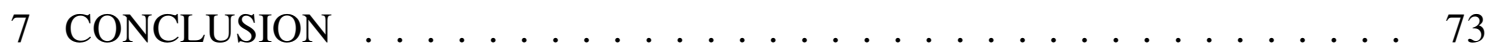

Appendix

A FLIGHT TEST DEVIATION RESULTS . . . . . . . . . . 75

B FLIGHT TEST MID POINT DEVIATION RESULTS . . . . . . . . . 75

REFERENCE LIST . . . . . . . . . . . . . . . . . . . . . . . 89

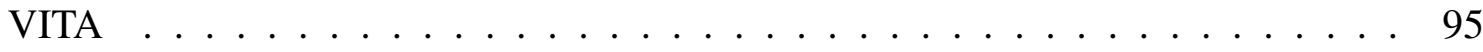




\section{ILLUSTRATIONS}

Figure Page

1 Server connections of HP DPS-1200FB-A switching power supply _ . . 22

2 Second generation UAS prototype ............... 31

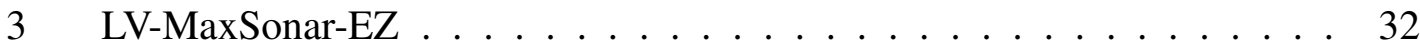

4 SparkFun MAX7456 Breakout Board . . . . . . . . . . . . 33

5 Normal plot of the effects .................. 36

6 Main effects plot for lift . . . . . . . . . . . . . 37

7 Interaction plot for lift . . . . . . . . . . . 38

8 Pareto chart of the effects . . . . . . . . . . . . 38

9 Fully equipped Octocopter ................... . . 42

10 High level $12 \mathrm{~V}$ power system schematic. . . . . . . . . . . . . . 44

11 Cost function results for copper (16 AWG) and copper-clad aluminum (16 AWG) wire. . . . . . . . . . . . . . . . . . 46

12 Fracture-critical, structurally deficient Boone County bridge. . . . . . . . 47

13 Tethered aircraft during superstructure inspection. . . . . . . . . . . . 48

14 Camera footage from tethered aircraft during substructure inspection. . . 49

15 Commercial-off-the-shelf DJI Inspire 1 aircraft during hovering flight op-

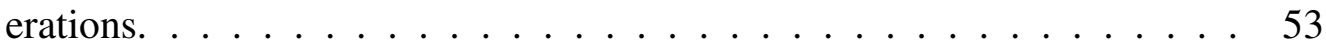

16 Steel construction with manual underexposure. . . . . . . . . . . . . 54 
17 Steel construction with manual overexposure. . . . . . . . . . . . . 54

18 Modified Cooper-Harper rating scale. . . . . . . . . . . . . . 56

19 Vertical $28 \mathrm{~cm}$ strip used for flight quality testing. . . . . . . . . . . 59

20 Horizontal $28 \mathrm{~cm}$ strip used for flight quality testing. . . . . . . . . . . 60

21 Horizontal $28 \mathrm{~cm}$ strip used for flight quality testing. . . . . . . . . . . 60

22 LabVIEW Vision Assistant screen shot. . . . . . . . . . . . . . . 62

23 Vertical strip pixel width at $1.5 \mathrm{~m}$ : Trial $1 \ldots \ldots 66$

24 Vertical strip pixel width at $3.0 \mathrm{~m}$ : Trial $1 \ldots \ldots 6$

25 Pixel width data inconsistencies. . . . . . . . . . . . . 67

26 Parking structure wall imperfections. . . . . . . . . . . . . 68

27 Vertical strip midpoint deviation at $1.5 \mathrm{~m}$ : Trial $1 \ldots \ldots$. . . . . . 70

28 Vertical strip midpoint deviation at $3.0 \mathrm{~m}$ : Trial $1 \ldots \ldots$. . . . . . . 70

A.1 Horizontal deviation at $1.5 \mathrm{~m}$ : Trial $1 \ldots \ldots \ldots$. . . . . . 75

A.2 Horizontal deviation at $1.5 \mathrm{~m}$ : Trial 2. . . . . . . . . . . 76

A.3 Horizontal deviation at $1.5 \mathrm{~m}$ : Trial $3 \ldots \ldots \ldots$

A.4 Horizontal deviation at $3.0 \mathrm{~m}$ : Trial 1. . . . . . . . . . . . . 77

A.5 Horizontal deviation at $3.0 \mathrm{~m}$ : Trial 2. . . . . . . . . . . 77

A.6 Horizontal deviation at $3.0 \mathrm{~m}$ : Trial $3 \ldots \ldots \ldots 78$

A.7 Vertical deviation at $1.5 \mathrm{~m}$ : Trial $1 \ldots \ldots \ldots$. . . . . . . 78

A.8 Vertical deviation at $1.5 \mathrm{~m}$ : Trial 2. . . . . . . . . . . . . . 79

A.9 Vertical deviation at $1.5 \mathrm{~m}$ : Trial 3. . . . . . . . . . . . . . 79

A.10 Vertical deviation at $3.0 \mathrm{~m}$ : Trial 1. . . . . . . . . . . . . . . 80 
A.11 Vertical deviation at $3.0 \mathrm{~m}$ : Trial 2. . . . . . . . . . . . . 80

A.12 Vertical deviation at $3.0 \mathrm{~m}$ : Trial 3 . . . . . . . . . . . . 81

B.1 Horizontal mid point deviation at $1.5 \mathrm{~m}$ : Trial $1 \ldots \ldots . \ldots 2$

B.2 VHorizontal mid point deviation at $1.5 \mathrm{~m}$ : Trial $2 \ldots \ldots . \ldots 3$

B.3 Horizontal mid point deviation at $1.5 \mathrm{~m}$ : Trial 3. . . . . . . . . . 83

B.4 Horizontal mid point deviation at $3.0 \mathrm{~m}$ : Trial $1 \ldots \ldots$. . . . . . . . 84

B.5 Horizontal mid point deviation at $3.0 \mathrm{~m}$ : Trial 2. . . . . . . . . . . 84

B.6 Horizontal mid point deviation at $3.0 \mathrm{~m}$ : Trial $3 \ldots \ldots$. . . . . . . 85

B.7 Vertical mid point deviation at $1.5 \mathrm{~m}$ : Trial $1 . \ldots \ldots 5$

B.8 Vertical mid point deviation at $1.5 \mathrm{~m}$ : Trial $2 \ldots \ldots . \ldots . \ldots 6$

B.9 Vertical mid point deviation at $1.5 \mathrm{~m}$ : Trial $3 . \ldots \ldots$. . . . . . 86

B.10 Vertical mid point deviation at $3.0 \mathrm{~m}$ : Trial $1 . \ldots . . . . . . . .887$

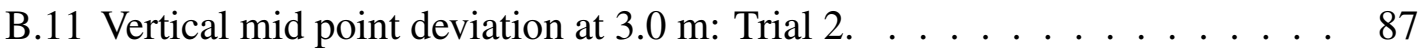

B.12 Vertical mid point deviation at $3.0 \mathrm{~m}$ : Trial $3 . \ldots \ldots$. . . . . . . 88 


\section{TABLES}

Tables

Page

1 AWG Specifications . . . . . . . . . . . . . . . . 24

2 Preliminary Tether Testing Results . . . . . . . . . . . . . 27

3 Preliminary Tether Testing Results . . . . . . . . . . . . . . . 28

4 Factorial experiment factors \& levels . . . . . . . . . . . . . 34

5 Lift Results with differing factors . . . . . . . . . . . . . . 35

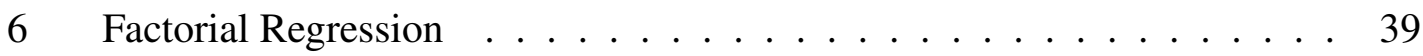

7 Strip widths ........................... 63

8 Horizontal flight Cooper-Harper rating . . . . . . . . . . . 63

9 Vertical flight Cooper-Harper rating . . . . . . . . . . . 63

10 Vertical-flight line pixel widths . . . . . . . . . . . . . 67

11 Horizontal-flight line pixel widths . . . . . . . . . . . . . 67

12 Horizontal flight marker mid point . . . . . . . . . . . 71

13 Vertical flight marker mid point . . . . . . . . . . . . 71 


\section{List of Abbreviations}

\begin{tabular}{|c|c|c|}
\hline Symbol & Units & Description \\
\hline$D_{A l}$ & $\mathrm{~m}$ & Copper-clad aluminum conductor diameter \\
\hline$D_{C u}$ & $\mathrm{~m}$ & Copper conductor diameter \\
\hline$g$ & $\mathrm{~m} / \mathrm{s}^{2}$ & Gravity of earth \\
\hline$I$ & A & Tether current \\
\hline$J$ & - & Tether power system cost function \\
\hline$L$ & $\mathrm{~m}$ & Total tether length \\
\hline$L_{A l}$ & $\mathrm{~m}$ & Total copper-clad aluminum tether length \\
\hline$L_{C u}$ & $\mathrm{~m}$ & Total copper tether length \\
\hline$P$ & - & Penalty function determined by tether constraints \\
\hline$P_{\text {load }}$ & $\mathrm{W}$ & Power drawn by aircraft \\
\hline$R$ & $\Omega$ & Total tether resistance \\
\hline$V_{\text {wall }}$ & $\mathrm{V}$ & Voltage sent to tether from VAC source \\
\hline$W$ & $\mathrm{lb}$ & Total tether weight \\
\hline$\rho_{A l}$ & $\mathrm{~kg} / \mathrm{m}^{3}$ & Aluminum density \\
\hline$\rho_{C u}$ & $\mathrm{~kg} / \mathrm{m}^{3}$ & Copper density \\
\hline$\rho_{e, A l}$ & $\Omega / \mathrm{m}$ & Aluminum resistivity \\
\hline$\rho_{e, C u}$ & $\Omega / \mathrm{m}$ & Copper resistivity \\
\hline
\end{tabular}




\section{ACKNOWLEDGEMENTS}

I would like to thank my academic advisor Dr. Travis Fields who has played a major role in all of my research and for all of his guidance throughout the last two years.

I would like to thank second advisor throughout this process Dr. John Kevern for his help and support.

I would like to thank the University of Missouri-Kansas City Students Engaged in Artistic and Academic Research (SEARCH) and the School of Graduate Studies for their funding. I would also like to acknowledge Gary Strack, Shafer, Kline \& Warren Inc., and the Missouri Department of Transportation for their assistance and allowing me to assist in their bridge inspection.

I would like to thank my family for making this possible. 


\section{CHAPTER 1}

\section{INTRODUCTION}

Bridges are a valuable part of infrastructure and travel that allow for the steady flow of traffic across rough terrain. Bridges offer a convenient method of connecting land and provide crossings over rivers, other roads, train tracks, and valleys. Due to the nature of bridges being suspended it is necessary to regularly asses their health. A bridge collapse will certainly be catastrophic and may cause severe injury and even death to the commuters who rely on them everyday.

Bridge maintenance encompasses several tasks that require both time and effort. Bridge inspectors need to carefully examine joints, fittings, small pockets and look for structural defects such as cracks, rust, and decay and monitor their progress. The process of inspecting needs to occur frequently due to the exposure of bridges to the elements and the stress and strain that is constantly placed on them.

This project describes an approach for multirotor-based inspection that increases capabilities and identifies the particular challenges associated with performing actual inspection operations. Four successive UAS inspection platforms were built with the third being used as an aide in an actual bridge inspection and the fourth being refined to meet the challenges experienced by the third. The first two prototypes were exploratory and used to identify essential hardware necessary to power an aircraft, capture images and video, and relay flight information to the pilot. The third developed multirotor aircraft 
system is equipped with an onboard high power AC-to-DC converter tethered to a standard gasoline generator. The power converter system is nearly the same weight as the standard flight battery for the aircraft developed for this study. The high voltage alternating current $(\mathrm{AC})$ power is transmitted up the tether from the generator to the onboard power supply. The power supply then converts the AC power to aircraft friendly lowvoltage DC power (12V). The higher voltage AC power can be delivered more efficiently across long distances as compared to low DC voltage because of the reduced current necessary to produce the same power (compared to low-voltage/high-current power transmission). An onboard camera coupled with an inspector-controlled stabilization gimbal is used to record video of inspector defined areas of interest. The video is transmitted in high-definition live to the inspector for real-time feedback. The high resolution of the video enables the inspector to identify areas of concern that can be examined in greater detail through post-processing of the video data. The fourth developed inspection platform is equipped with an onboard high power DC-to-DC converter tethered to a ground stationed AC-to-DC converter. The DC-to-DC system is $50 \%$ lighter than the aircraft battery and receives high DC voltage from the tether. The high DC voltage experiences relatively small voltage drop allowing for a longer tether. The fourth UAS allows for remote camera control giving the option to increase and decrease camera exposure.

The project further describes the development of a new metric used for measuring UAS handling. A modified Cooper-Harper rating scale tailored specially for UAS is used to asses the handling of the fourth multirotor inspection system. Aircraft handling is further explored by measuring deviation from an inspection target while in flight. 


\section{Motivation}

More than ten percent of the nation's bridges are currently rated as structurally deficient, with a current overall bridge grade of $\mathrm{C}+$ as rated by the American Society of Civil Engineers [1]. Federal law mandates call for biennial inspections (Title 49 C.F.R. 650.311). However, due to lack of monetary resources bridges often go uninspected beyond the time allotted by federal regulations [1]. Cost of an inspection can vary significantly from bridge to bridge as size, type, and location determine machinery, workforce, and time needed to complete an inspection. For many large bridges an adjustable inspection vehicle (snooper truck) is used to maneuver under the bridge for visual inspection of the substructure. Small bridges are often too small to support heavy snooper trucks and require inspection crews to use scaffolding, ladders, and climbing harnesses to make a visual determination. The setup necessary for scaffolding and ladders can further elongate inspection times to several days for a single bridge. Additionally, for the safety of the public, bridges may become partially or completely closed off to traffic.

An alternative to traditional inspection techniques includes the assistance of a UAS. Unmanned aircraft have been used in the areas of research, surveying, scanning, mapping, and transportation [2-4]. The benefits of UAS in agriculture has been investigated in which different UAS equipped with remote sensors gathered thermal, multispectral, and hyper-spectral data from crop fields [5]. Data from the onboard sensors was compared to ground-collected plant data to provide indications of vegetation health. Light unmanned aircraft have also seen use in the area of land surveying, particularly archeological sites, and performing photogrammetry [6]. Unmanned aircraft have also been 
used to perform inspections of building facades that are often difficult to reach especially with ground-based monitoring devices [7]. The applicability of autonomous unmanned aircraft for bridge inspections has also been investigated [8]. However, the applicability for bridge inspections is typically performed only in simulation. The high variability in bridge geometry, surface effects (i.e. bird feces), and environmental variations creates an extremely difficult scenario for autonomous inspections. Bridge inspections require the vehicle to be capable of examining small pockets throughout the structure. Although autonomous inspections may be theoretically possible, the widespread use of autonomous vehicles is still many years away. Remotely controlled systems require a pilot to operate the vehicle; however, the pilot can maneuver the aircraft in a variety of ways to ensure quality data is captured during inspections.

UAS missions often span vast amounts of time requiring constant battery replacement. The task of battery replacement becomes inefficient if the aircraft needs to cover long distances. Travel to and from an inspection location severely cuts down on valuable battery power. The most efficient UAS batteries only provide fifteen to twenty mintues of flight time; if an inspection location is too far away from the pilot, trying to monitor it with a fifteen minute flight will be useless. If it takes ten minutes to reach a destination then the aircraft will require immediate return since it will only have five to ten minutes left of flight time.

One approach to removing battery limitations is through the use of a powered tether. Recently, Wang et. al. developed a methodology for the selection of an optimal tether capable of providing sufficient power to maximize lifting capacity [9]; however, 
the technique was only created in simulation. A $8 \mathrm{~m}(26 \mathrm{ft}$.$) tethered powered UAS ap-$ plicable to civilian tasks has been developed while also focusing on a reeling mechanism that senses tension and slack [10]. Previously developed tethered power systems offer unlimited flight time but send low-voltage (12-24V) direct current power through the tether, thereby substantially limiting the achievable tether length.

\section{Thesis Objectives}

The aim of this project is to determine if tethered unmanned aircraft systems (UAS) are a viable aid in the inspection of bridges. Determining the applicability of UAS technology to bridge inspections requires meeting the following objectives:

1) Develop UAS tether enabling unlimited flight time.

2) Conduct field testing with tethered system to gain fundamental knowledge of inspection process.

3) Quantify aircraft flight handling in stressful scenarios.

\section{Thesis Organization}

The organization of this thesis is as follows: Chapter 2 contains the literature review and outlines uses of UAS including for bridge inspections, previous efforts to create a power tethered UAS, and the process of bridge inspections. Chapter 3 presents the development of the first generation prototype. The first generation prototype was very simple in design but served as an outline as to the hardware (both onboard and for the powering system) that would be necessary for successful flight. Chapter 4 describes the 
development of the second generation prototype and the process taken to maximize thrust capabilities by altering hardware configuration. Chapter 5 describes the development of the third generation prototype and its assistance in the completion of a bridge inspection. Chapter 6 describes the development of the fourth generation prototype built in response to feedback provided by bridge inspector's experience with the third generation system along with flight handling testing and results. Conclusions and summary are presented in Chapter 7. 


\section{CHAPTER 2}

\section{BACKGROUND \& LITERATURE REVIEW}

\section{Visual Bridge Inspection Background}

Bridge inspections are vital to the preservation of safe infrastructure. As bridges get older they become more vulnerable to deterioration making it necessary to regularly assess their condition. Frequent inspections are needed to identify structural issues that need repair to prevent them from becoming safety hazards. Federal law mandates that bridges be inspected once every twenty-four months (Title 49 C.F.R. 650.311).

More than ten percent of the nation bridges are currently rated as structurally deficient [1]. The high rate of deficient bridges is due to inpsections occuring less frequently. The decrease in inspections is due to a lack of monetary resources and funding [1]. The Federal Highway Administration (FHWA) estimates that $\$ 20.5$ billion in funding should go towards bridge maintenance. Currently, only $\$ 12.8$ billion is going towards bridge maintenance [1].

Bridge inspections are costly due to the high volume of equipment, personnel, and machinery needed to conduct a safe and timely inspection. For the safety of motorists and the inspection crew, it is often necessary to establish traffic control which may require partial or complete closure of the bridge. Partial closure often results in lane closures and the need for personnel to guide approaching motorists. Large equipment is needed primarily when inspecting the sides, superstructure, and substructure of the bridge. Safety 
harnesses and lanyards, ladders, scaffolding, riggings, climbers, floats, manlifts, truckmounted man-lifts, scissors lift, platform trucks, bucket trucks, and underbridge inspection vehicles with either platforms or buckets are all optional equipment to asses bridge elements [11]. The actual visual inspection process is quite archaic and does not require special hardware. Inspectors will position themselves within arm's length of the bridge and subjectively assess bridge structure health [12]. Areas of concern are photographed and included in inspection reports for future inspections. Inspectors qualitatively judge the progression of damage (if any) while providing a rating and then report their findings.

\section{Early Unmanned Aerial Systems and Their Applications}

The first recorded unmanned aerial systems were mostly balloons and were employed for several purposes but particularly for war. The first recorded UAS launched from a ship were kites launched from aboard a Royal Navy frigate in 1806 to distribute propaganda leaflets [13]. Decades later on August 22, 1849 Austria flew two hundred balloons, armed with 33 pounds of explosives, over the city of Venice. The balloons measured approximately twenty-three feet in diameter and relied heavily on wind for steering [13]. Wind effects were not always favorable as some of the balloons reached their target while the majority were blown back towards Austria. The failure of bombing by balloon lead to the Austrians abandoning the use of explosive-carrying-balloons [14]. In the United States, balloons were introduced in 1793 and utilized for reconnaissance by the Union Army in 1861 [13]. The following year Luther C. Crowell of Massachusetts patented a winged aircraft capable of carrying explosives. The aircraft was designed with 
movable wings and propellers allowing for vertical and horizontal steering and was capable of vertical takeoff and landing [13]. In 1863 Charles Perley of New York patented a hot-air balloon bomber with a timing mechanism which released a bomb from the bottom of the balloon [13]. Following the use of balloons driven by wind, engineers began developing methods of controlling aircraft through wireless communication.

\section{UAS Applications in Surveying, Sensing, \& Scanning}

Unmanned aircraft has been used extensively in inspections, search and rescue, surveying and remote sensing [15-18]. They have also found signifant use by the military $[19,20]$. In "Low-Cost Aerial Unit for Outdoor Inspection of Building Facades" the authors used a UAS equipped with a Microsoft Kinect sensor in order to collect geometric data from buildings [7]. A building's geometry directly influences energy performance and must be measured when inspecting building energy. A UAS was preferred due to the complex nature of using terrestrial devices for examining high and complex locations. While photogrammetry and laser scanning are often times performed singularly or in conjunction with one another they do not provide full detail of a building due to their incapability to reach high locations. The authors state that these issues can be solved by using UAS which can gather digital imagery and then generate 3D point clouds. The authors chose to use a Kinect sensor due to its versatility and that it is capable of capturing both photographic and geometric data. The authors provide a solution as to the acquisition of data and its generation of point clouds. The authors describe the configuration of the aerial unit along with the 3D sensor, data acquisition, and point cloud generation. A 
concrete facade in Vigo, Spain served as the testing sample and used a HiSystems GmbH eight propeller copter. The octocopter was chosen due to its greater lift capabilities, recovery from motor failure, low cost, and maneuverability.

In "Aerial Laser Inspection of Building Facades Using Quadrotor", the authors attempted to bring together aerial vehicles and commonly ground based laser scanning devices. The authors expected the concept to provide detection of building facade defects while limiting the time and range of facade laser scanning [21]. In the process of building face inspections, laser scanning generates 3D models based on still images. These images were used in determining the state of quality of scanned buildings. Therefore, the quality of the picture is a high priority. Weather plays a significant role in the life of buildings. Harsher weather conditions contribute to quicker deterioration. As a result, frequent facade inspection is necessary. From the ground, laser scanning devices are used to identify any significant cracks that may be present in building facades. However, ground stations present significant impediments that make quick and accurate detection of deteriorating areas problematic. Laser scanning devices have a defined scanning range which require heavy human interaction in order to relocate devices once a specific area has been scanned. another method of face inspection is photogrammetry which involves the assessment of the building structure through still photographs [21]. While the reliability of photogrammetry lies in its accuracy laser scanning faces significant height issues. In conjunction with laser scanning, photogrammetry has proven to be a powerful tool in assisting in facade inspections. Since photogrammetry is normally done aerially, the authors suggest combining the two methods into an aerial platform capable of creating 3D 
models and photographs. The authors list three main methods of scanning civil structures and determine the advantages and disadvantages of each. Tachometry requires 3 days of fieldwork, 1 day of topographic planning, and 4-person-minimum team. A manned aircraft requires 96 hours of traversing and flight planning which will require up to 1 month of image scanning and processing. A manned aircraft requires two pilots and at least one ground person. Operating a UAS requires one person, 20 minutes of aircraft setup, 30 minutes of flight, and only 4 hours of image processing. The authors determined that using a UAS is the most viable and cost friendly option. However, the work identifies one prevalent issue when operating a UAS: stability. The authors go onto providing a theoretically stable quadrotor.

The complexity of power line detection and tracking offers itself as a viable candidate for UAS application [22-24]. Zhengrong et. al. developed a UAS platform to relieve the effort and expense of managing vegetation that builds around electrical components. The UAS operated autonomously relying on algorithms to distinguish power lines from background images. Zhang et. al. also developed an autonomous vehicle while extracting and filtering captured images to identify power lines [22]. Jones also presented a concept of an inspection platform but had it powered through the power lines themselves. The UAS consisted of a single ducted fan that remains in contact with the power lines at all times while it navigates around the structure [25].

Surveying, sensing, and scanning as conducted by the mentioned works had the benefit of flying UAS in large and open-air spaces where the aircraft had the ability to obtain a GPS lock. The applied UAS were also battery powered significantly limiting 
flight time. The surveying and scanning performed differs from bridge inspections in that inspecting bridges require maneuvering around large and complex steel and concrete structures that can prohibit GPS lock and interfere with aircraft compass orientation. Nevertheless, the aforementioned research show the when equipped with the necessary image capturing and sensing hardware, UAS can effectively perform tasks that require direct human interaction.

Attempts at prolonging UAS flight were recently explored by Fujii et. al. The research team developed a landing platform that automatically changed aircraft batteries without the need for human interaction [26]. The battery replacement platform was designed for use in surveillance, monitoring, and any other task that would require a UAS to cover large areas. The UAS was powered through a typical LiPo battery providing approximately ten minutes of flight time. A low battery triggered the aircraft to land autonomously on the platform where a mechanism replaced the battery. Preliminary testing showed that the mechanism could successfully switch aircraft batteries without human interaction and shows possibilities of being used in field testing. The mechanism developed by Fujii et. al. has its limitations in that placement of battery stations would still require human interaction. The mechanism would be difficult to implement in hard-to-reach areas such as disaster sites where human access is significantly limited.

\section{UAS for Bridge Inspections}

UAS have recently started to be used in the area of bridge inspections [27] [28] [29] [30] [31]. Two years ago when this thesis project began, very little could be found 
on the subject of using UAS for bridge inspections. Today, multiple institutions have published preliminary findings of using UAS for bridge inspections. Multiple state departments of transportation (DOT), sometimes in collaboration with university research groups, have found success in using multirotor platform inspection units. In 2008 the California Department of Transportation (Caltrans) presented a report on attempts to construct a UAS to examine difficult-to-access structural components [30]. The unit consisted of a ducted fan to generate lift capable of vertical takeoff and landing and was tether-powered. The unit consisted of a camera capable of transmitting video to a ground station. Caltrans developed a functioning flying aerial robot with necessary onboard hardware for inspecting but due to impending issues could not deployed within the allotted time. Some of the intended powering and controlling mechanisms did not perform to Caltrans standards and was not used for bridge inspections. Although not implemented, Caltrans did successfully construct a single-duct aerial robot with a $200 \mathrm{ft}$ tether with a 10-15 lb payload capacity equipped with image and video capturing hardware [30].

Multirotor UAS are ideal due to their hovering and abilities to fly in confined spaces. In 2013 the Michigan and Georgia DOTs investigated techniques to analyze bridge elements using UAS [32]. The Florida Institute of Technology (FIT) completed a two year study using UAS for high mast pole and bridge inspections in 2015 [33]. In 2015 the Minnesota DOT also published their findings after using UAS for inspecting multiple bridges throughout the state [31]. At the Missouri University of Science \& Technology (Missouri S\& T), researchers developed an autonomous bridge inspecting UAS platform 
using non-invasive sensors to detect structural deficiencies [34]. FIT prepared their findings in an extensive report for the Florida DOT which highlighted the limitations of using UAS for inspections. UAS performance was examined through altitude, payload, first person view (FPV), and maneuverability tests [33]. FIT also identified maximum flight times for different aircraft with different hardware configurations. In addition to flight testing, FIT developed a simple flight training program to provide bridge inspectors with a general background in the concept of UAS, their handling and maneuverability. Aircraft handling in high pressure wind was tested and revealed that the selected UAS could fly stably in $10 \mathrm{mph}$ winds at a distance of $1 \mathrm{ft}$ from an object. For wind speeds of 15 up to $20 \mathrm{mph}$ it was recommended that the aircraft be flown at a distance of at least $3 \mathrm{ft}$. Altitude testing showed that the aircraft remained visibly in-sight for up to $250 \mathrm{ft}$. Altitudes exceeding $250 \mathrm{ft}$ required the pilot to rely on ground station data to locate the aircraft. With the aid of an FPV system the pilot was able to control the aircraft at altitudes of up to $400 \mathrm{ft}$. Aircraft payload capabilities were identified taking into consideration propeller material, battery capacity, and attainable flight time. FIT's hexacopter platform had a flight time of 10 minutes when carrying a camera and water protective case. With an additional 1.5 and 2 lbs payload the hexacopter's flight time decreased to 8.23 and 7.52 minutes, respectively. Using carbon fiber propellers extended the hexacopter's flight time by 1.5 minutes. Additional testing indicated that the largest available battery $(22000 \mathrm{mAh}$ $2.8 \mathrm{lbs}$ ) provided 20.64 minutes of flight with a $1 \mathrm{lbs}$ payload capacity. Field testing included inspecting $90 \mathrm{ft}$ tall high mast luminaires (HMLs). The pilot was able to maintain a distance of $4 \mathrm{ft}$ from the light fixtures with wind speeds of approximately $10 \mathrm{mph}$ [33]. 
Closer flying distances were possible but under the piloting of a highly experienced operator. Field testing at a girder bridge presented difficulties when flying in confined spaces. Flying between girders produced turbulence making it difficult to achieve stable flight. Both a hexacopter and quadcopter were utilized for girder inspection with both aircraft experiencing the same results. Inspection of steel components resulted in identification of defects with image post-processing and zoom.

The Minnesota DOT recently used a multirotor inspection system to study its effectiveness in analyzing four bridges throughout the state [31]. Previously published inspection reports were used as a guide in examining previously identified structural deficiencies. The ability of the aircraft to identify previously identified deficiencies determined inspection system success. An off-the-shelf Skyranger UAS was used to inspect bridge superstructures, substructure, and decks [31]. Inspection of the substructure was avoided on some bridges due to the inability to obtain a GPS signal. The aircraft camera system and its zoom capabilities were sufficient in detecting deficiencies throughout the structures. The inspection system delivered strong results when inspecting a large complex steel bridge with a $185 \mathrm{ft}$ clearance previously requiring rope access to fully inspect.

The aerial systems used for bridge inspections have so far relied on batteries for power. The designed systems also had difficulty flying in GPS denied areas and in close proximity to large structures. FIT testing showed that their designed aircraft could stably fly within one to three feet of structures under variable wind conditions; however, when inspecting bridges the designed aircraft could not fly within reasonable distances and captured images required post-processing and zoom to examine bridge component 
details. Gathering of useful inflight data was difficult to obtain and the aforementioned UAS conducted only mock inspections and findings from aircraft inspections were not used for official bridge inspection reports.

\section{Tethered UAS}

The method of operating a tether-powered UAS has been implemented but with limitations. Surveying, scanning, inspecting, and research with tethered aircraft has has been done both as powering methods and to stay within US Federal Aviation Administration (FAA) requirements [35-37]. On December 6, 2007 the parking garage of the Berkman Plaza II condominium development in Jacksonville, Florida collapsed resulting in the death of one person [38]. Identifying the cause of the accident required overhead plan-view images of the construction site. Overhead images were also needed to asses the level of damage caused by the collapse. Using a manned helicopter caused excessive turbulence resulting in dust and debris kicking up and obstructing the view of the site; the proximity of the helicopter also caused safety concerns. Instead, surveying was done by the Center for Robot-Assisted Search and Rescue (CRASAR) which used a tethered iSensys IP3 miniature helicopter to perform three survey flights. Since February 6, 2007 FAA regulations have not permitted small unmanned aerial systems (sUAS) to fly in the United States National Airspace (NAS) unless a Certificate of Authorization (COA) has been obtained [38]. The CRASAR was not granted a COA since the miniature helicopter was not involved in search and rescue [38]. As a result, the miniature helicopter was attached to a $45 \mathrm{~m}(150 \mathrm{ft})$ tether to comply with FAA regulations. The inspection made 
by CRASAR was successful in providing structural engineers with sufficient overhead information. The tethered system presented multiple issues including the potential for the tether to snag and the obstruction caused by the tether when taking off. When taking off, the weight of the tether and its proximity to the propellers increased pilot workload as he had to focus on countering the tether weight and avoid hitting the tether.

The aforementioned tethered system used by CRASAR relied on batteries to power the sUAS. Powering a UAS through the tether itself has been proposed and developed. A tether powered UAS was developed that worked adjacently to an UGS (unmanned ground system) [39]. The UAS-UGS system was autonomous and navigated through environments where limited knowledge of the environment is known. To overcome the payload restrictions of the aircraft, the UGS was used to carry the power supply used to power the tether. The UAS was used to provide aerial coverage necessary for successful navigation [39]. The ground system ultimately dictated travel while the air system served as a navigator/scout. The dual vehicle system was successful in navigating through a controlled environment. The aircraft remained power throughout the prolonged process indicating that the tether and power supply successfully replaced an onboard battery.

A UAS similar to that developed by Papachristos and Tzes also aimed at achieving extended flight time by utilizing a tether. Power for the aircraft was drawn from a power supply located on the ground. The power supply fed an $8 \mathrm{~m}(26.2 \mathrm{ft})$ tether through a reeling mechanism that worked autonomously. The reel detected the tension through the tether during flight. Excessive tension caused the reel to loosen the tether while excessive slack caused the reel to retract [10]. The aircraft flew autonomously within a confined 
space and experienced occasional disturbances from the tether; the tether caused a noticeable opposing force resulting in the aircraft steering away from its intended path. Zikou et. al. concluded that the aircraft's stability was not critically affected by the disturbances as the reel was properly tuned and allowed for smooth release and retraction of the tether. Although the tether carried direct current the tether was short enough to where voltage drop across the line did not affect the aircraft's operation [10].

While many tether powered UAS have been developed in practice, there are also tethered UAS that have been developed in theory and simulation. Samarathunga et. al. designed a hexarotor with automated tether reeling and high payload capacity exceeding $30 \mathrm{~kg}(66.1 \mathrm{lb})$. The proposed powering system consisted of a power generator outputting 200V three phase AC. A 200VAC-to-380VDC power conversion unit powered a tether connected to a 380VDC-to-48VDC and a 380VDC-to-24VDC power converter each for DC motors and autopilot, respectively [40]. Thrust test results indicated that using $29 \mathrm{x}$ 9.5 in CF propellers, each motor produced more than $10 \mathrm{~kg}$ of lift at $80 \%$ throttle. The reeling mechanism was automated and utilized a servo motor. The reel design included tension sensors to avoid excessive tension by retracting and loosening the tether when necessary [40]. A method of modeling a deployed tether was investigated.

Currently there exists a number of companies that offer tethered drone services to the public. Companies such as $\mathrm{CyPhy}^{\mathrm{TM}}$, Polarity ${ }^{\circledR}$, and Hoverfly ${ }^{\circledR}$ currently offer aerial drone video services capable of reaching altitudes of atleast 150 feet. Hoverfly's LIVESKY ${ }^{\mathrm{TM}}$ tether-powered UAS is an $\mathrm{AC}$ powered quadrotor with a standard tether height of 150 feet, 1080p 60fps HD video, $360^{\circ}$ aircraft/camera rotation, and PTZ with 
10x optical zoom. LIVESKY utilizes a battery backup system, is capable of auto takeoff/landing, provides continuous in-flight diagnostics and monitoring, and has a payload capacity of $2.2 \mathrm{lbs}(1 \mathrm{~kg})$. Customers have the option of adding weather proof enclosure, IR thermal imaging camera, thermal imager FLIR, and tetracam multispectral imaging camera. CyPhy's Persistent Aerial reconnaissance and Communication (PARC ${ }^{\mathrm{TM}}$ ) unit is a hexcopter capable of operating at $400 \mathrm{ft}(0.122 \mathrm{~m})$ with a payload capacity of $2 \mathrm{lb}(0.9$ $\mathrm{kg}$ ). The aircraft can withstand continuous wind speeds of 25 knots and gusts of up to 35 knots. Like LIVESKY, PARC also relies on battery for backup emergency landings. The Polarity TPS500-2.0kw offers quad, hexa, and octocopters capable of operating at $500 \mathrm{ft}$ and also relies on battery backup for emergency landing due to tether power loss. The PARC $4 \mathrm{k}$ camera can rotate $360^{\circ}$ and comes with the option of adding an IR camera. 


\section{CHAPTER 3 \\ FIRST GENERATION PROTOTYPE Introduction}

This chapter covers the approach taken to develop the first generation prototype capable of performing bridge inspections. An off-the-shelf UAS platform and autopilot were selected as a base for the prototype. Selections were made from a variety of available multi-rotor drones. An AC/DC converter took in AC from a tether and was used to deliver 12 VDC to the unmanned aircraft simulating a 3-cell lithium polymer battery, but enabling unlimited flight time. An battery backup system was developed to permit a safe aircraft landing in the event of an AC/DC converter failure. Initial testing was conducted to identify a suitable conductor material and size for the powering tether. Power consumption was measured for each wire size to determine the appropriate gauge required to develop the tether.

\section{System Development}

\section{UAS Development}

The first drone inspection prototype was developed as simply as possible while still capable of demonstrating viability for inspection operations. The aircraft would have to carry the power supply, tether, camera, LED light for navigating in dark settings, and an optimal power backup system. A Turnigy hexcopter was chosen as the platform for 
the first generation prototype. The chassis was made of carbon fiber, offering a higher strength-to-weight ratio than the more common commercially available plastic frame. The drone was equipped with six Turnigy G10 Brushless Outrunner 810kv motors and $11 \times 4.7$ inch propellers constructed out of ABS plastic. Flight stabilization is performed with an off-the-shelf multirotor autopilot system (DJI NAZA-M V2 ${ }^{\mathrm{TM}}$ ). The aircraft was remotely controlled through a Spektrum DX6i. Six Turnigy Plush 40A electronic speed controllers were used. The camera platform consists of a 3-axis stabilization gimbal (DJI Zenmuse $^{\mathrm{TM}}$ ) and a HD camera (GoPro Hero3 ${ }^{\mathrm{TM}}$ ) mounted on top of the aircraft frame.

\section{Power System Development}

The power supply used to provide continuous power to the vehicle was selected based on the powering needs of the selected aircraft platform and hardware. The power converter was developed using an HP Switching Power Supply model DPS-1200FB A. The power supply is rated for an input voltage of 110-120VAC and 200-240VAC and an input current of 10A and 7.8A, respectively. With an input of 110-120VAC and 200240VAC the power supply can output 12.0 VDC and a maximum current of $75 \mathrm{~A}$ and $100 \mathrm{~A}$, respectively. The power supply is intended for use in computer server systems. To use as a lone power supply the effects of being connected to a server needed to be mimicked. By installing a 330 to $1000 \Omega$ resistor between two of the terminals [41]. A $1000 \Omega$ resistor was soldered between the terminals labeled 33 and 36 (Figure 1). 


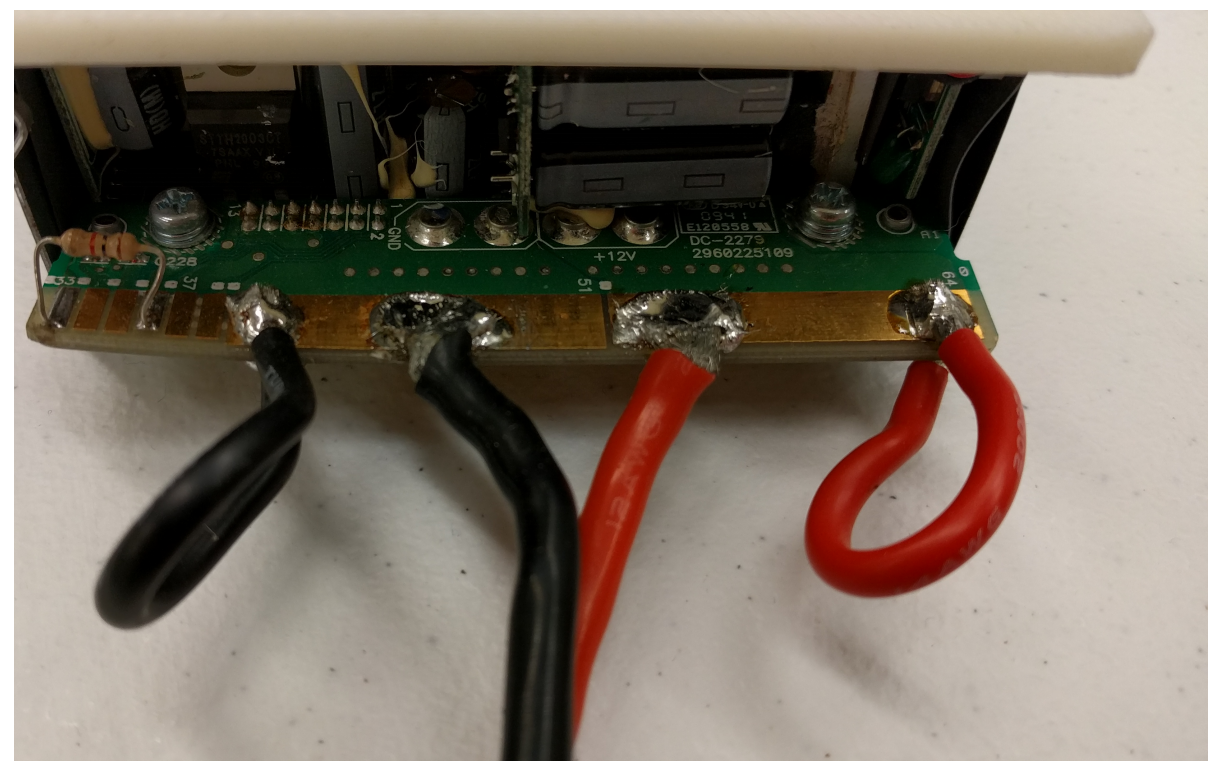

Figure 1: Server connections of HP DPS-1200FB-A switching power supply

\section{Battery Backup System Development}

Keeping in mind that safety for all personnel is of greatest concern, a backup powering system was developed. In case of potential power supply failure without the backup system the aircraft would descend rapidly out of control and potentially cause severe harm to personnel and/or property. Since the chance of electrical component failure is possible a backup powering method was implemented. The backup system consists of a 3-cell lithium-ion polymer battery, diodes, relay, and microcontroller. The backup system detects the absence of voltage being delivered from the power supply to the aircraft and onboard components. The lack of voltage triggers a mechanism that would allow the flow of voltage from an onboard LiPo battery to the aircraft and all onboard hardware. The battery does not substitute the power supply but merely provide the pilot enough time to 
fly the aircraft back and land in a safe location. From there any inspection and repair to the power supply could be made.

A solid state relay (SSR), Arduino Mini, and 12 VDC LiPo battery were used for the battery backup system. The board was programmed using the Arduino software. Power from the power supply was sent to the A/D input on the board. The board monitors the analog signal. If and when the power supply ceases to deliver 12 volts to the analog pin, the board sends 5 volts to a digital pin. The pin outputs a voltage that is sent to the SSR. The SSR input is connected to an LED which illuminates across an air gap. The light is detected by a sensor which is connected to the transistors which open and close delivering power to the load. When the transistor is closed current is allowed to run through the relay. When the transistor is open nearly all current is blocked. The SSR was chosen over a mechanical relay or a simple switch due to the high current that is being drawn. SSRs are able to control high-current devices with low current signals. To protect the SSR a diode was connected across the load. The diode protects against strong residual currents after transistors are turned off. This precaution is necessary due to the buildup of magnetic fields around wiring by inductance while a load is being powered. As the SSR is turning off magnetic fields push current against the SSR and damage in the absence of a protective diode. The diode allows for the recirculation of currents in the load until their energies dissipate. Is a diagram of the backup battery system with the UAV and power supply. 


\section{Tether Development \& Testing Methodology}

The next phase of the system development involved identifying an appropriate gauge size for the tether wire. A series of power consumption experiments were performed on tether samples. A power test was designed and constructed using two tether lengths of three differing gauge sizes. The American Wire Gauge (AWG) standard of measuring wire size was used (1). A lower AWG size indicates a larger conductor diameter. While heavier, a larger conductor will lead to less voltage drop and less tether heating. A higher AWG size indicated a smaller conductor diameter. A smaller conductive material would lead to greater voltage drop and overheating but would result in a lighter tether.

\begin{tabular}{ccc}
\multicolumn{3}{c}{ Table 1: AWG Specifications } \\
\hline AWG & Ohm/km & $\mathbf{m O h m / f t}$ \\
\hline 10 & 3.277 & 0.09988 \\
12 & 5.211 & 0.1588 \\
14 & 8.286 & 0.2526 \\
16 & 13.17 & 0.4014 \\
18 & 20.95 & 0.6386 \\
20 & 33.31 & 1.0153 \\
22 & 52.96 & 1.6142 \\
24 & 84.22 & 2.5670 \\
26 & 133.9 & 4.0813 \\
28 & 212.9 & 6.4892 \\
30 & 338.6 & 10.3205 \\
\hline
\end{tabular}


Testing was performed using three different AWG sizes each of two different lengths. AWG sizes 24, 22, and 20 of lengths 18 in and 24 in were used. The tether was connected between a 120VAC power outlet and the AC-DC converter. Current was measured at both ends of the tether: as power was transferred to the tether from the power outlet and as power was transferred to the power converter. Temperature readings were collected at both ends of the tether. The end of the tether connected to the power outlet was designated location $A$ while the opposite end of the tether connected to the power converter was designated location $B$. Temperature readings of the six motors and power converter were also recorded. The aircraft was fastened down to a tripod test stand. The test stand was weighed down to assure that the aircraft remain stationary during test. Full throttle setting was used for testing as full throttle requires maximum power. Power supply failure along with tether overheating and melting were indicative of a failed system. All readings were taken at two different time intervals (300 and 600 seconds). Three trials were performed for each AWG size.

\section{Test Results \& Discussion}

Test results are presented in Tables 2 and 3. Six trials were conducted for each AWG size with the first three trials presented in Table 2 and the last three trials presented

in Table 3. Results demonstrate that when at full throttle the power consumption of the aircraft was within the power supply standards of 10 A. Although warm the tether was able to provide the the AC-DC converter with sufficient power without overheating. No power supply failures were recorded indicating that all three AWG sizes would provide 
the UAS with the necessary power required to operate at full throttle. Based on the results a larger AWG size was chosen. Although 24 AWG looked promising and is lighter, 20 AWG was chosen since the larger wire causes less voltage drop. The decision was made to construct a tether running $60 \mathrm{ft}$ long using $20 \mathrm{AWG}$ size copper wire. The tether was expected to provide the aircraft with the necessary power. Attempting to operate the UAS with such a tether resulted in power supply failure as the power consumption proved to be too high resulting in full AC-DC converter shut-off. The aircraft was operating at half throttle when failure occurred. The aircraft hovered for approximately 10 seconds before experiencing failure. It was thought that the 20 AWG wire would compensate for voltage drop experienced by 24 AWG. Voltage drop across the $60 \mathrm{ft} 20$ AWG tether exceeded expectations. A viable tether could not be constructed with the powering method and required immediate modifications. 
Table 2: Preliminary Tether Testing Results

\begin{tabular}{ccccccccc}
\hline AWG & Time (s) & Current (A) & \multicolumn{6}{c}{ Temperature (C) } \\
\hline & & & $18 ”$ & $24 ”$ & A & B & Motor & P.S. \\
24 & 300 & 3.16 & 25.5 & 25.1 & 26.3 & 25.5 & 41.3 & 33.4 \\
24 & 600 & 3.11 & 25.5 & 25.4 & 26.7 & 26.4 & 47.4 & 47.3 \\
24 & 300 & 3.16 & 25.4 & 24.7 & 25.7 & 24.9 & 46.5 & 48.5 \\
24 & 600 & 3.14 & 25.1 & 24.6 & 25.5 & 25.9 & 47.0 & 53.3 \\
24 & 300 & 3.15 & 25.9 & 25.8 & 27.6 & 26.3 & 44.4 & 51.3 \\
24 & 600 & 3.14 & 26.3 & 26.8 & 27.5 & 26.5 & 42.0 & 54.0 \\
22 & 300 & 3.10 & 25.5 & 25.7 & 26.7 & 26.1 & 45.5 & 44.3 \\
22 & 600 & 3.09 & 24.9 & 24.6 & 26.3 & 25.4 & 45.4 & 47.5 \\
22 & 300 & 3.18 & 25.4 & 25.7 & 26.7 & 25.6 & 50.9 & 51.0 \\
22 & 600 & 3.04 & 24.9 & 25.2 & 26.4 & 25.8 & 44.5 & 53.6 \\
22 & 300 & 3.13 & 25.5 & 25.9 & 26.9 & 26.0 & 47.1 & 56.0 \\
22 & 600 & 3.14 & 25.5 & 25.5 & 26.8 & 25.2 & 43.2 & 56.1 \\
20 & 300 & 3.17 & 26.2 & 26.0 & 27.6 & 25.8 & 45.7 & 53.1 \\
20 & 600 & 3.05 & 26.3 & 25.9 & 27.4 & 25.6 & 45.5 & 54.6 \\
20 & 300 & 3.06 & 26.4 & 25.7 & 27.5 & 25.0 & 45.4 & 56.0 \\
20 & 600 & 3.06 & 26.1 & 25.5 & 27.4 & 25.3 & 45.9 & 54.3 \\
20 & 300 & 3.06 & 27.1 & 26.5 & 26.4 & 25.9 & 42.7 & 56.0 \\
20 & 600 & 3.04 & 26.4 & 25.9 & 27.3 & 25.8 & 45.1 & 54.5 \\
\hline & & & & & & & &
\end{tabular}


Table 3: Preliminary Tether Testing Results

\begin{tabular}{ccccccccc}
\hline AWG & Time (s) & Current (A) & \multicolumn{6}{c}{ Temperature (C) } \\
\hline & & & $18 ”$ & $24 ”$ & A & B & Motor & P.S. \\
24 & 300 & 4.08 & 26.1 & 26.1 & 25.7 & 25.5 & 32.5 & 44.7 \\
24 & 600 & 4.05 & 25.0 & 25.1 & 24.5 & 24.5 & 32.5 & 51.3 \\
24 & 300 & 4.05 & 25.5 & 25.2 & 25.7 & 25.0 & 33.4 & 50.3 \\
24 & 600 & 4.01 & 24.8 & 24.7 & 24.8 & 24.5 & 32.8 & 53.1 \\
24 & 300 & 4.05 & 25.9 & 25.7 & 25.6 & 25.1 & 33.5 & 53.7 \\
24 & 600 & 4.02 & 24.8 & 25.0 & 25.0 & 24.8 & 33.6 & 53.1 \\
22 & 300 & 4.14 & 26.4 & 26.1 & 28.6 & 26.1 & 32.8 & 52.0 \\
22 & 600 & 4.10 & 25.3 & 24.6 & 26.0 & 25.5 & 32.2 & 52.0 \\
22 & 300 & 4.14 & 26.0 & 26.0 & 26.5 & 26.0 & 34.0 & 53.2 \\
22 & 600 & 4.07 & 25.3 & 25.1 & 26.5 & 25.3 & 33.4 & 50.9 \\
22 & 300 & 4.11 & 25.6 & 25.6 & 25.9 & 25.5 & 33.8 & 53.3 \\
22 & 600 & 4.02 & 25.3 & 25.3 & 25.1 & 25.3 & 34.0 & 51.4 \\
20 & 300 & 4.18 & 25.8 & 26.1 & 25.8 & 24.6 & 34.0 & 53.3 \\
20 & 600 & 4.08 & 25.8 & 26.1 & 25.7 & 24.9 & 34.1 & 51.8 \\
20 & 300 & 4.12 & 26.5 & 28.1 & 28.5 & 26.0 & 34.3 & 54.1 \\
20 & 600 & 4.08 & 26.0 & 27.8 & 27.0 & 25.8 & 34.3 & 53.0 \\
20 & 300 & 4.26 & 27.7 & 26.5 & 25.9 & 26.5 & 31.5 & 43.3 \\
20 & 600 & 4.12 & 27.1 & 26.5 & 26.4 & 26.2 & 33.3 & 51.5 \\
\hline & & & & & & & &
\end{tabular}

\section{Conclusion}

Based on test results the aircraft would not be able to function properly given the set of motors and power supply being used. To generate sufficient lift the motors require more power given the tether length tested. The power supply failed when using a 60 
$\mathrm{ft}$ tether and a $60 \mathrm{ft}$ tether is not even sufficient for many bridge inspection operations. Any tether length greater than $60 \mathrm{ft}$ will lead to an even great voltage drop which will require even more current to provide the aircraft with the necessary power. Based on the insufficient tether capabilities, a new set of motors within acceptable power limitations was needed. A new set of motors needed to be capable of generating more lift with less current than that required by the first generation motors. The first generation prototype motors used were rated specifically for the aircraft platform used. New motors would require a new UAS. 


\section{CHAPTER 4}

\section{SECOND GENERATION PROTOTYPE}

\section{Introduction}

This chapter describes the development of the second generation prototype. The second generation prototype built upon the first generation by building upon the lessons learned and overcoming the limitations. The new aircraft is equipped with new motors capable of generating sufficient thrust while remaining within power converter current limits. Development of the battery backup and onscreen dsiplay systems are also introduced. This chapter also addresses efforts to maximize aircraft lift capabilities through selection of appropriate hardware (propeller size, propeller material, and AC-DC converter). ANOVA testing was conducted to identify the necessary hardware. This chapter concludes by analyzing flight capabilities of the UAS and identifying its shortcomings.

\section{System Development}

\section{Aircraft Platform Development}

The second generation UAS prototype was built upon the Turnigy S1000 hexcopter frame (Figure 2). The aircraft was intended to also carry the same power supply, camera, LED light, tether, and power backup system from the first generation. Based on the failure of the power converter in the first generation prototype phase testing was conducted to ensure that the aircraft was capable of takeoff with all necessary hardware 
onboard (or equivalent weight). In addition, the aircraft had to be capable of safely hovering while at approximately 50\% full throttle. Hardware configuration and produced lift were important in selecting an appropriate flight apparatus. The second generation chassis was also made of carbon fiber. The drone was equipped with six AX-2810Q-750KV brushless motors and $11 \times 4.7$ propellers constructed out of ABS plastic. The aircraft was remote controlled through the same Spektrum DX6i while a DJI M-V2 autopilot was used to ensure stable flight. Six Turnigy 10A electronic speed controllers were used.

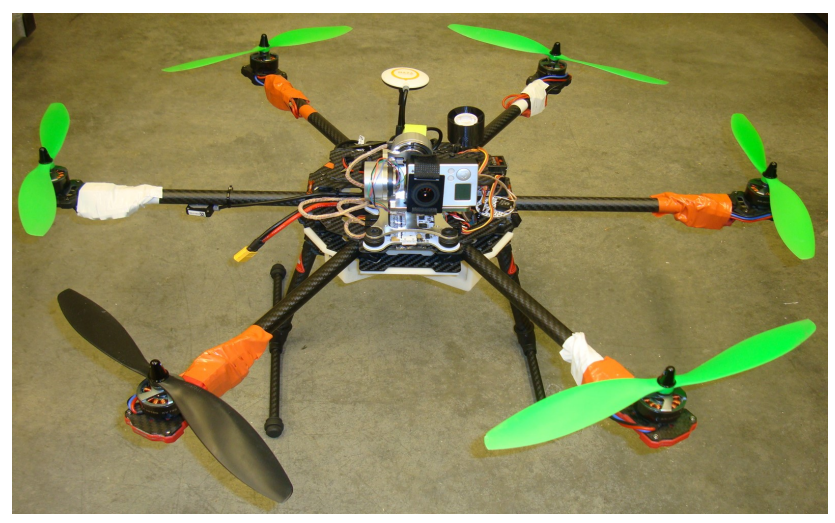

Figure 2: Second generation UAS prototype

Sensor \& OSD System Development

For the safety of all personnel taking part in bridge inspections, the backup battery system was developed; however, there needed to be a method for the ground crew to safely monitor the state of the power system. In case of power supply failure, the battery backup would take over and provide the aircraft and onboard hardware with the necessary amount of power. However, with the first generation aircraft the pilot would have no method of monitoring and noticing if and when such a failure occurred. Therefore, an onscreen 
display system was developed that would allow the flight crew to monitor voltage levels of the system in real time. In addition, the pilot would need to be aware of obstructions being monitored by the onboard sensors (Figure 3). Distance measurements made by the sonar sensors would be relayed to the pilot through the OSD system.

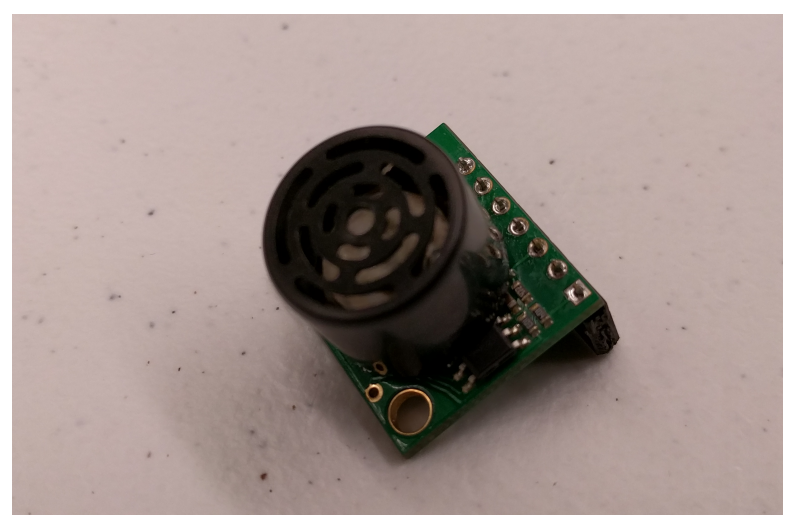

Figure 3: LV-MaxSonar-EZ

The OSD system overlays text over video transmitted to the grounded pilot and inspector. Since the same video feed to the ground monitor and goggles is used, it is convenient for both monitoring devices to have overlay sensor measurements. Both the voltage and distance measurements were monitored by an Arduino microcontroller. The microcontroller allows for simple integration of OSD hardware. For the OSD system, a Sparkfun MAX7456 breakout board was used (Figure 4). The breakout board consisted of two video connections, one for receiving and the other for transmitting. The received video was taken directly from the onboard video camera (GoPro Hero 3+). The breakout board was programmed to overlay certain information received from the microcontroller. Sensor information was then overlayed onto the live video feed and transmitted to the 
ground station and goggles.

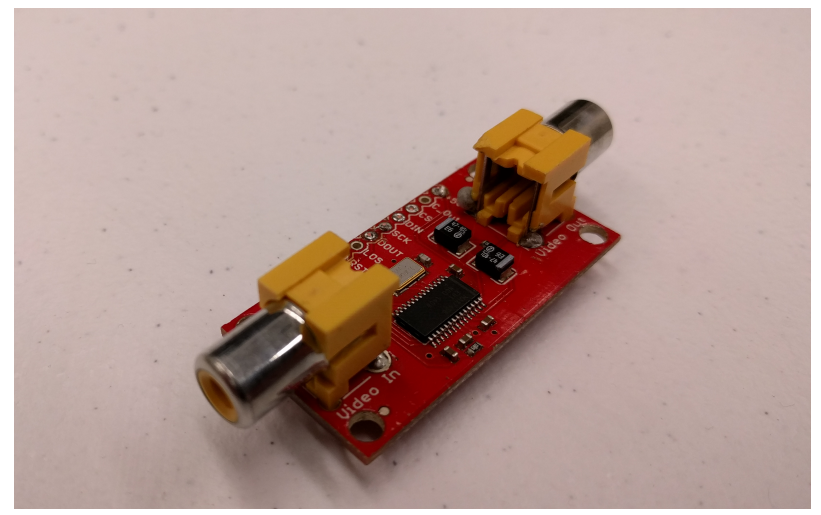

Figure 4: SparkFun MAX7456 Breakout Board

\section{Thrust Maximization Testing}

The UAV needed to be capable of hovering and ascending while carrying loads of up to eight pounds consisting of: the camera, gimbal, power supply, and tether. To resolve the issue of voltage drop across the tether a higher voltage of $220 \mathrm{VAC}$ was sent to the onboard power converter. Four factors effect lift capabilities including: propeller material, propeller size, motors, and power converter/supply. A factorial experiment was conducted to determine the appropriate hardware configuration to produce maximum lift. Factorial designs were used to explore all possible hardware combinations of the four factors and their generated lift. A $2^{k}$ factorial design was used to determine which configuration provides maximum lift capabilities. 


\section{Testing Methodology}

The hexcopter was mounted on a test stand and connected to a load cell. The load cell measured force while in tension. A National Instruments data acquisition system was used to acquire data from a load cell and record in a LabView VI. Since maximum lift was of interest the hexacopter was set to full throttle. Four factors were determined to be most significant in affecting lift and ran at two levels. The levels were arbitrarily called low (-) and high (+). Factor A was assigned to propeller material and the two levels of interest were ABS plastic and carbon fiber. The propeller size was designated factor B, with the low level designating 11 inch propellers and the high level designating 12 inch propellers. The power supply was factor C, with the two levels of interest being the DPS 1200 and the DS 1600 . The motor was factor D, with $810 \mathrm{kv}$ motors denoted by the high level and $750 \mathrm{kv}$ motors denoted by the low level. The two levels for each of the four factors are summarized in Table 4:

Table 4: Factorial experiment factors \& levels

\begin{tabular}{cc|c}
\hline \multicolumn{2}{c|}{ Level } & Factor \\
\hline+ & - & \\
\hline Carbon Fiber & ABS & Propeller Material \\
12 in & $11 \mathrm{in}$ & Propeller Size \\
DS 1600 & DPS 1200 & Power Supply \\
$810 \mathrm{kv}$ & $750 \mathrm{kv}$ & Motor \\
\hline
\end{tabular}

\section{Test Results}

The design matrix and lift generated from two trials of the $2^{4}$ experiment are shown in Table 5. All possible combinations of the four factors were tested. In Table 
5, the two columns labeled Trial 1 and Trial 2 contain the lift generated by the respective hardware configuration. The last column under Lift contains the sample average of the lift measurements for both trials.

Table 5: Lift Results with differing factors

\begin{tabular}{cccc|ccc}
\hline \multicolumn{3}{c|}{ Factor } & \multicolumn{3}{c}{ Lift $(\mathrm{kg})$} \\
\hline $\mathrm{A}$ & $\mathrm{B}$ & $\mathrm{C}$ & $\mathrm{D}$ & Trial 1 & Trial 2 & Average \\
\hline- & - & - & - & 3.85 & 3.91 & 3.88 \\
+ & - & - & - & 3.71 & 3.72 & 3.72 \\
- & + & - & - & 3.43 & 3.40 & 3.42 \\
+ & + & - & - & 3.36 & 3.29 & 3.33 \\
- & - & + & - & 3.25 & 3.25 & 3.25 \\
+ & - & + & - & 3.16 & 3.13 & 3.15 \\
- & + & + & - & 2.89 & 2.87 & 2.88 \\
+ & + & + & - & 2.74 & 2.78 & 2.76 \\
- & - & - & + & 4.76 & 4.74 & 4.75 \\
+ & - & - & + & 4.68 & 4.63 & 4.66 \\
- & + & - & + & 4.18 & 4.20 & 4.19 \\
+ & + & - & + & 4.10 & 4.09 & 4.10 \\
- & - & + & + & 4.01 & 3.97 & 3.99 \\
+ & - & + & + & 3.88 & 3.87 & 3.88 \\
- & + & + & + & 3.53 & 3.55 & 3.54 \\
+ & + & + & + & 3.45 & 3.46 & 3.46 \\
\hline
\end{tabular}

The experiment was replicated twice leading to thirty two total runs (thirty two lift measurements). It was appropriate to consider the lift measurements as duplicate measurements and not as replicates. Had each lift measurement been taken on a single run then they would be actual replicates. Therefore, the average of the lift measurements was the appropriate response variable to consider leading to sixteen actual runs. Normal plots and Pareto Charts were used to identify any significant factors and/or interactions. The model was then modified, if necessary, to determine important factors and interactions. 
The statistics package Minitab was used to perform an analysis of variance (ANOVA) of the collected lift results for each factor combination to identify variations among generated lift. Before performing an ANOVA the test model was examined to identify the effect of the four different factors on the response (lift). The effect of a factor is the change in lift resulting from a change in the level of a factor. Analysis of a normal probability plot of these effects is required. Figure 5 shows both negligible and large effects. The negligible effects lie along the line of best fit while the large effects lie far away from the line. From the normal plot it is seen that main effects of A, B, C, and D along with interactions $\mathrm{BC}, \mathrm{BD}, \mathrm{CD}$ are significant effects. Another option in determining the significant effects comes from the Pareto chart shown in Figure 8. Based on the Pareto chart, effects of A, $\mathrm{B}, \mathrm{C}, \mathrm{D}, \mathrm{BC}, \mathrm{BD}$, and $\mathrm{CD}$ are deemed significant. This is determined by the point of demarcation (0.0434) between significant and non-significant effects.

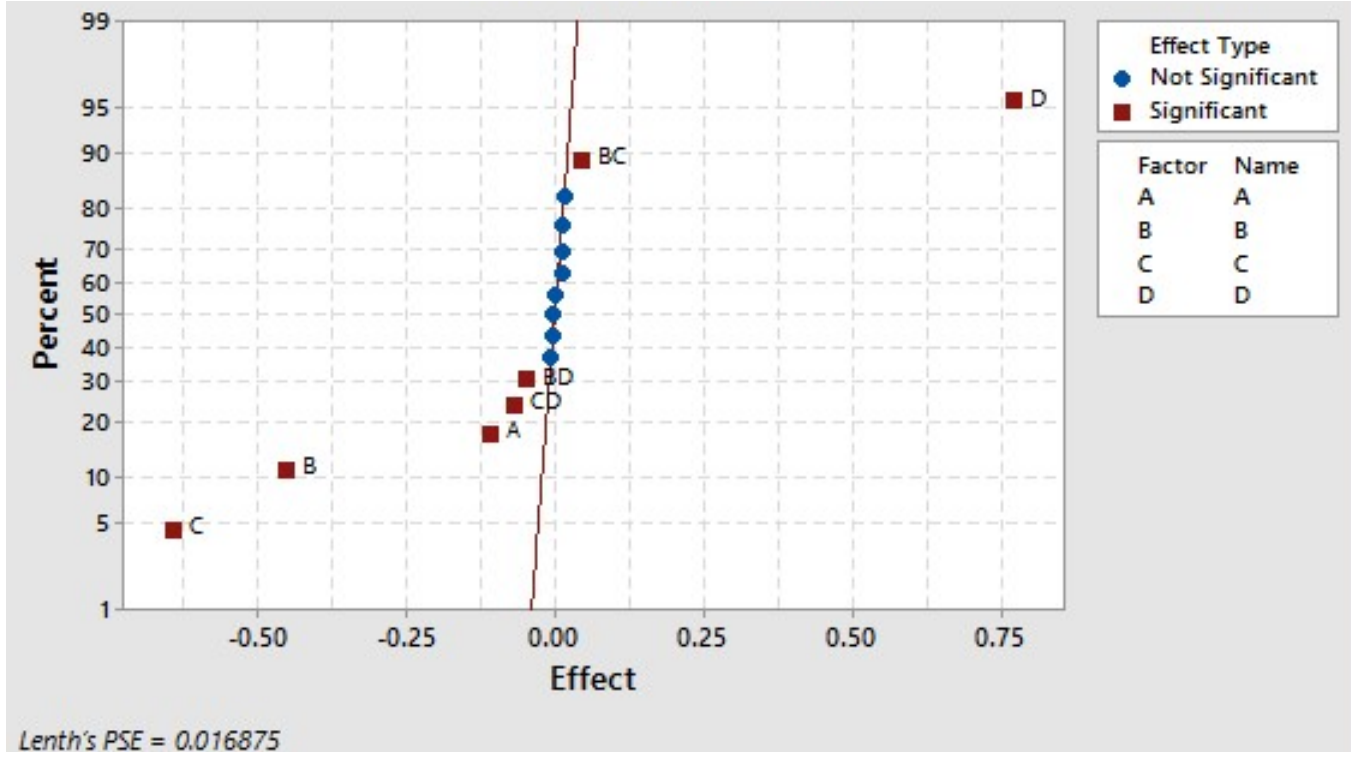

Figure 5: Normal plot of the effects 
The main effects of A, B, C, and D are plotted in Fiure 6. Effects of factors A, B, and $\mathrm{C}$ are negative indicating that to generate maximum lift, factors $\mathrm{A}, \mathrm{B}$, and $\mathrm{C}$ should be ran at low levels (11 in ABS props with DPS 1200 power supply). Main effect D is positive indicating that to generate maximum lift factor D should be ran at the high level (810 kv motors). Apart from effects it is necessary to examine important interactions. The $\mathrm{BD}, \mathrm{CD}$, and $\mathrm{BC}$ interactions from the normal plot are plotted in Figure 7. The BC interaction plot shows that the low level propeller size has greater effect on lift with the low level power supply. The BD interaction plot shows that the low level propeller size has greater effect on lift with the high level motor. The CD interaction plot shows that the low level power supply has greater effect on lift with the high level motor. Therefore, maximum lift is achieved when $\mathrm{A}, \mathrm{B}$, and $\mathrm{C}$ are at the low level and $\mathrm{D}$ is at the high level.

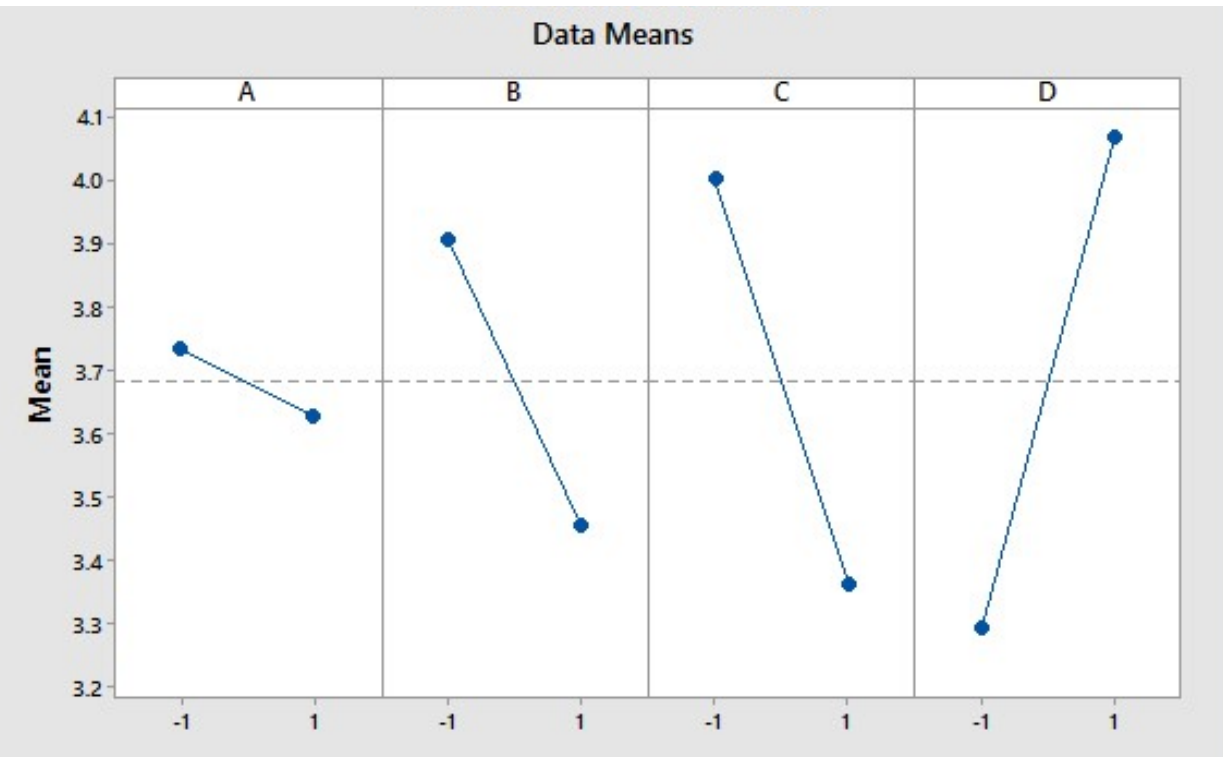

Figure 6: Main effects plot for lift 


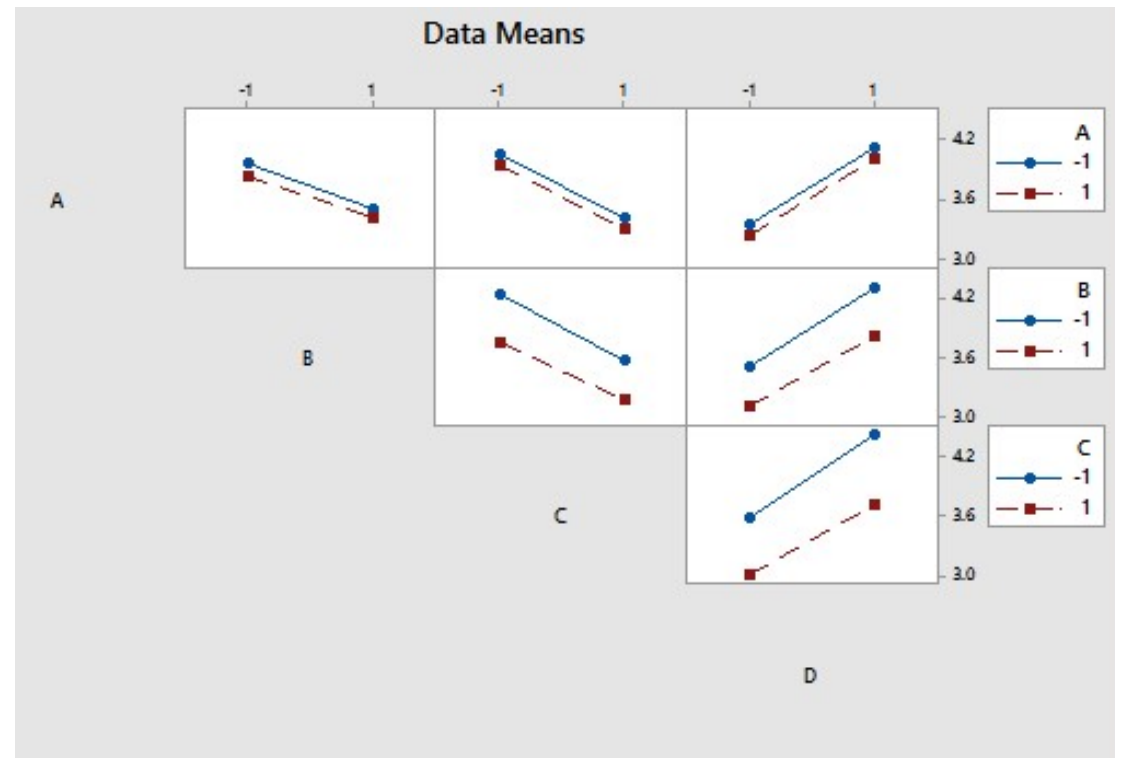

Figure 7: Interaction plot for lift

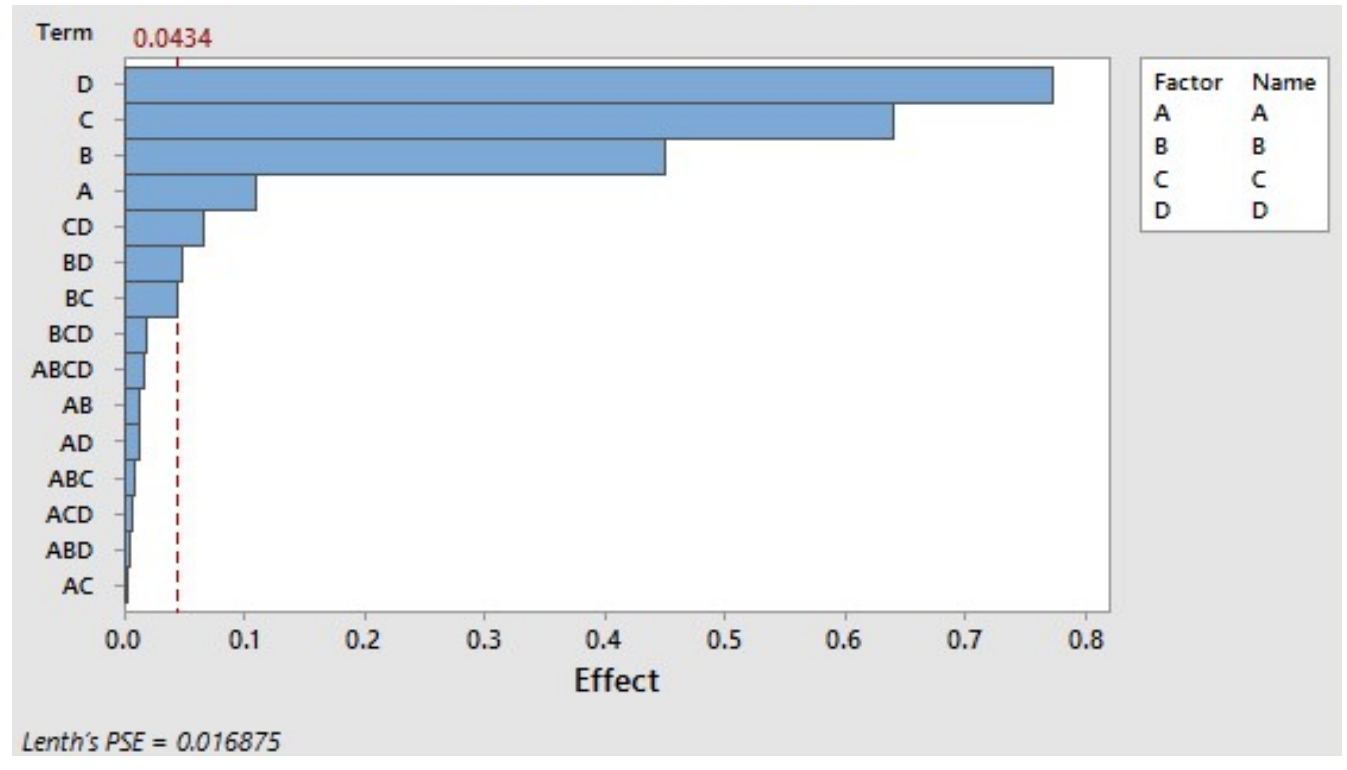

Figure 8: Pareto chart of the effects

With the identification of significant effects, the model was refined to factors A, B, 
$\mathrm{C}$, and $\mathrm{D}$ and interactions $\mathrm{BC}, \mathrm{BD}$, and $\mathrm{CD}$. An ANOVA was performed using the refined model. The results from the analysis are shown in Table 6. Noticing that the $\mathrm{P}$ value for all factors and all interactions is below 5\% (0.05) it is determined that prop material, prop length, power supply, and motor along with the interaction between length and power supply, length and motor, and power supply and motor are important and affect hexacopter lift. The model has an R square value of $99.86 \%$ indicating that the fitted model is highly accurate. Therefore, the model is deemed adequate and supportive of the assumption that maximum lift is generated by using factors $\mathrm{A}, \mathrm{B}$, and $\mathrm{C}$ at the low level (11 in ABS props with DPS 1200 power supply) and D at the high level (810 kv motors).

Table 6: Factorial Regression

\begin{tabular}{cccccc}
\hline Source of Variation & Sum of Squares & DOF & Mean Square & F-Value & P-Value \\
\hline A & 0.047 & 1 & 0.047 & 101.6 & 0.0 \\
B & 0.81 & 1 & 0.81 & 1739.6 & 0.0 \\
C & 1.64 & 1 & 1.64 & 3532.5 & 0.0 \\
D & 2.39 & 1 & 2.39 & 5126.5 & 0.0 \\
B C & 0.00766 & 1 & 0.0076 & 16.44 & 0.004 \\
$\mathrm{~B}^{*} \mathrm{D}$ & 0.00903 & 1 & 0.00903 & 19.38 & 0.002 \\
$\mathrm{C}^{*} \mathrm{D}$ & 0.0176 & 1 & 0.0176 & 37.7 & 0.0 \\
Error & 0.00373 & 8 & 0.00047 & & \\
Total & 4.93 & 15 & & & \\
\hline
\end{tabular}

\section{Conclusion}

The first generation prototype failed at generating sufficient thrust necessary to carry the onboard hardware, power supply, and small tether. Due to hardware configuration, the first generation aircraft experienced excessive power consumption causing power supply failure. Factorial design was used to identify the hardware configuration 
that produced maximum lift. The second generation prototype was equipped with eleven inch-ABS plastic propellers, $810 \mathrm{kv}$ motors, and the DPS 1200 power supply. The aircraft did not exceed power supply limits and successfully carried the $60 \mathrm{ft}$ tether. During flight testing the aircraft experienced repeated undesired yawing due to motor saturation resulting in excessive current draw and overheating; saturation prevented the motor from operating efficiently. The aircraft was recoverable but the defect caused a severe safety concern. The motor saturation was attributed to power consumption that was not correctable through motor replacement. Therefore, a third prototype was developed capable of operating efficiently with the power supply and producing sufficient lift. 


\section{CHAPTER 5}

\section{THIRD GENERATION PROTOTYPE}

\section{Introduction}

This chapter describes the development of the third generation prototype. An eight propeller aircraft was chosen as the platform for the third generation. The aircraft was equipped with new motors capable of producing more lift and requiring less current than the two previous sets of motors used for generations 1 and 2. The third generation utilized significantly larger props than those used previously in order to provide significantly greater thrust. An optimization approach was created to determine the appropriate conductor size and material to create the powering tether. The aircraft was able to fly stably with the tether system and did not experience power supply failure. A bridge inspection was performed following development of the third prototype. Feedback from bridge inspectors was used to identify inspection system shortcomings and areas that required improvement.

\section{System Development}

The third generation inspection aircraft (shown in Figure 9) is an eightrotor vehicle in the four-arm configuration or X8 configuration (two counter-rotating rotors on each arm) with a system weight (excluding battery/power supply weight) of 3.5

$\mathrm{kg}(7.7 \mathrm{lb}$.$) . Each motor has a lift capability of 1.4 \mathrm{~kg}(3.1 \mathrm{lb}$.) resulting in a maximum 
$(<65 \%$ maximum lift) power system and payload weight of approximately $0.91 \mathrm{~kg}(2.0$ lb.) with $12 \mathrm{~V}$. The $X$ configuration prevents the aircraft from achieving its maximum potential lift due to the counter-rotating nature of the rotors. Load cell validation showed that the aircraft produced only $78 \%$ of the maximum theoretical lift. The maximum lift generated by the aircraft is $8.7 \mathrm{~kg}(19.1 \mathrm{lb})$ with $12 \mathrm{~V}$. Most flight occurs at hover $(70 \%$ maximum throttle) meaning that the $\mathrm{X} 8$ can lift a maximum payload of $2.6 \mathrm{~kg}(5.7 \mathrm{lb})$.

Flight stabilization is performed with the same DJI Naza m v2 autopilot. The gimbal and flight control commands are combined and transmitted over a wireless telemetry link (DJI Lightbridge ${ }^{\mathrm{TM}}$ ). Additionally, the telemetry link also transmits the onboard high-definition (HD) video to the operator(s). The camera platform consists of a 3-axis stabilization gimbal (DJI Zenmuse ${ }^{\mathrm{TM}}$ ) and a HD camera (GoPro Hero4 ${ }^{\mathrm{TM}}$ ) mounted on top of the aircraft frame. Finally, the video data is viewed from both HD goggles (Cinemizer) and on a high resolution computer monitor (ASUS).

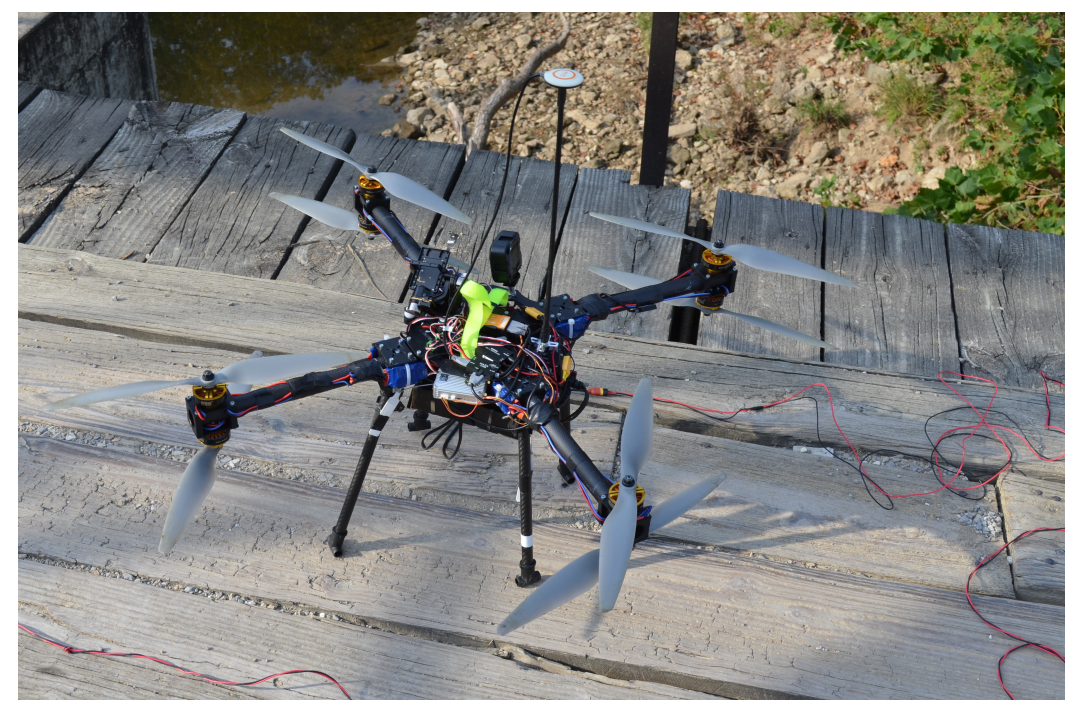

Figure 9: Fully equipped Octocopter 
To improve the lift capabilities of the previous two prototypes within the power system constraints, an octocopter frame was assembled in the X8 configuration. The increased rotors enabled higher total thrust capable with minimal increase in weight as the X8 configuration has only four arms compared to the six arms necessary for the hexcopter design. The eight rotor system utilizes all of the available $1.2 \mathrm{~kW}$ from the power supply at maximum throttle. A variety of rotor sizes and shapes were evaluated to yield the maximum lift achievable without damaging the power system. After the maximum thrust data was collected the top rotor candidates were evaluated qualitatively via free-flight testing to ensure flight stability.

\section{Tether \& Power Systems Development}

For the tethered aircraft to be feasible, the tether and power system had to be both lightweight and have a small footprint. Server power supplies provide high power output $(1.2 \mathrm{~kW})$ at a standard $12 \mathrm{VDC}$ in a standardized footprint that resembles a large lithium polymer (LiPo) battery. The power supply measures $(8.6$ x 3.9 x $20.6 \mathrm{~cm})$ and weights $1.1 \mathrm{~kg}$. An input voltage of 220VAC was utilized to improve maximum output capabilities and reduce the voltage drop associated with 110VAC power. A patent pending battery backup system was also created and implemented to provide short duration flight capabilities in the presence of a tether system failure. The $12 \mathrm{~V}$ tether system is shown in Figure 10.

Voltage drop proved to be a major concern from preliminary flight testing. The significant drop in voltage caused a higher current draw exceeding power supply current 


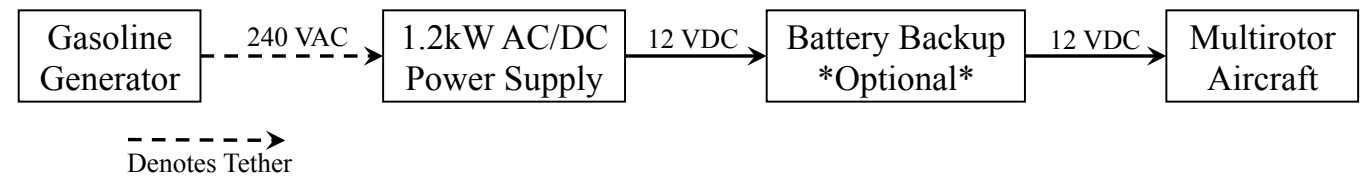

Figure 10: High level 12V power system schematic.

limitations of $10 \mathrm{~A}$. The octocopter requires $600 \mathrm{~W}$ of power to hover with full payload (power supply, camera, gimbal, tether, etc.). Testing was conducted with a copper-coated aluminum 18 AWG $500 \mathrm{ft}$ tether. With a 120 VAC source the voltage experienced a drop of $42.8 \mathrm{~V}$. To reach the necessary $600 \mathrm{~W}$ the octocopter needed to draw $10.4 \mathrm{~A}$. An appropriate tether length can not be found analytically as the length is constrained by the minimum acceptable input voltage to the power supply, tether weight, and tether conductive material. For example, as the tether wire diameter increases (to reduce the tether voltage drop), the weight also increases. An optimization approach was created to identify the best tethering system within a specific set of constraints. The cost function selected for minimization is:

$$
J=W^{2}+I^{2}-L+R^{2}+P
$$

Where $J$ is the cost function, $W$ is the tether weight, $I$ is the current requirement, $L$ is the tether length, $R$ is the tether resistance, and $P$ is the penalty function determined by tether constraints. Two different wire materials (copper-coated aluminum and copper) of four different AWG sizes ranging from $20(0.812 \mathrm{~mm})$ to $14(1.628 \mathrm{~mm})$ were tested. Pure copper has the benefit of having better conductive qualities but is heavier than coppercoated aluminum. The components of the cost function are determined with the equations 
below.

$$
\begin{gathered}
L=L_{A l}+L_{C u} \\
W=\pi g D_{A l}^{2} L_{A l} \rho_{A l}+\pi g D_{C u}^{2} L_{C u} \rho_{C u} \\
R=\frac{8 \rho_{e, A l} L_{A l}}{\pi D_{A l}^{2}}+\frac{8 \rho_{e, C u} L_{C u}}{\pi D_{C u}^{2}} \\
I=V_{\text {wall }}-\frac{\sqrt{V_{\text {wall }}^{2}-4 P_{\text {load }} R}}{2 R}
\end{gathered}
$$

Based on available payload the maximum tether weight was not to exceed $1.5 \mathrm{~kg}$ (3.3 lb) and minimum power supply input voltage was set to 190VAC (per manufacturer supplied data). The penalty function was set to 100 for each constraint violated to ensure clear violation of the design constraints. The input variables of the multivariate optimization were copper-coated aluminum wire diameter and length, and copper wire diameter and length. Due to the relative simplicity of the equations, direct parameter space investigation was conducted. A representative surface plot for the relationship between the cost function and the two wire lengths is shown in Figure 11. For this surface the coppercoated aluminum wire has a diameter of $1.291 \mathrm{~mm}(0.0508 \mathrm{in}$.$) and the copper wire also$ has a diameter of $1.291 \mathrm{~mm}$. Within the constraints identified, a tether length of $55 \mathrm{~m}$ (180 ft.) was achieved. The final tether consists of $22 \mathrm{~m}$ of 16 AWG copper and $33 \mathrm{~m}$ of 16 AWG copper-coated aluminum wire. 


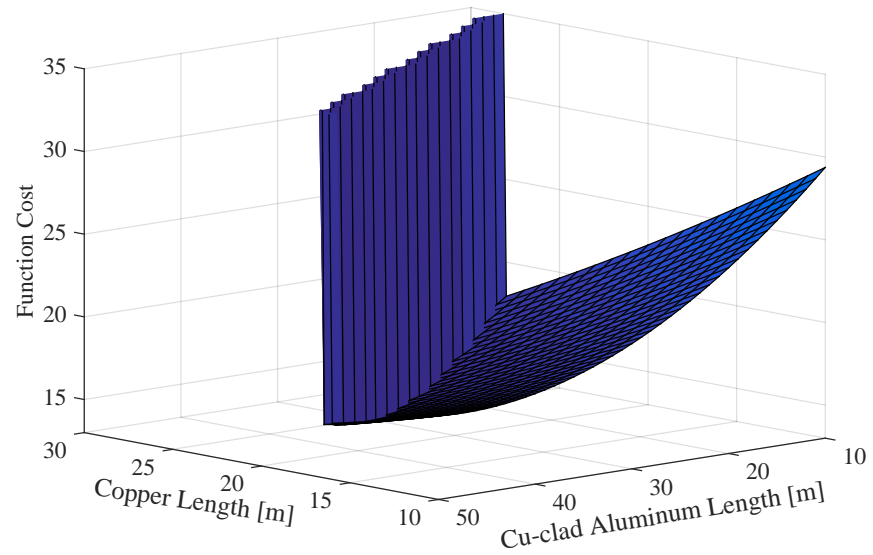

Figure 11: Cost function results for copper (16 AWG) and copper-clad aluminum (16 AWG) wire.

\section{Inspection Flight Test Methodology}

The inspection platform was evaluated through field testing in collaboration with the Missouri Department of Transportation inspection crews. An example of the bridges inspected is shown in Figure 12. The fracture-critical bridge is located in Boone County, Missouri and is one of many structural deficient bridges in Missouri. The objectives of the field testing were to: 1) show stability of the aircraft in proximity to the bridge, 2) determine limitations imposed by tether-based inspections, 3) evaluate the video (both live stream and post-processed) data and compare with traditional inspection data, and 4) determine the overall feasibility of a tethered drone used for bridge inspections.

Testing was conducted in two phases: 1) bridge superstructure inspection and 2) bridge substructure inspection. The areas of main concern on the superstructure were the connection locations. The aircraft was flown around the various connections while the inspector manipulated the gimbaled camera. A crew member monitored the tether 


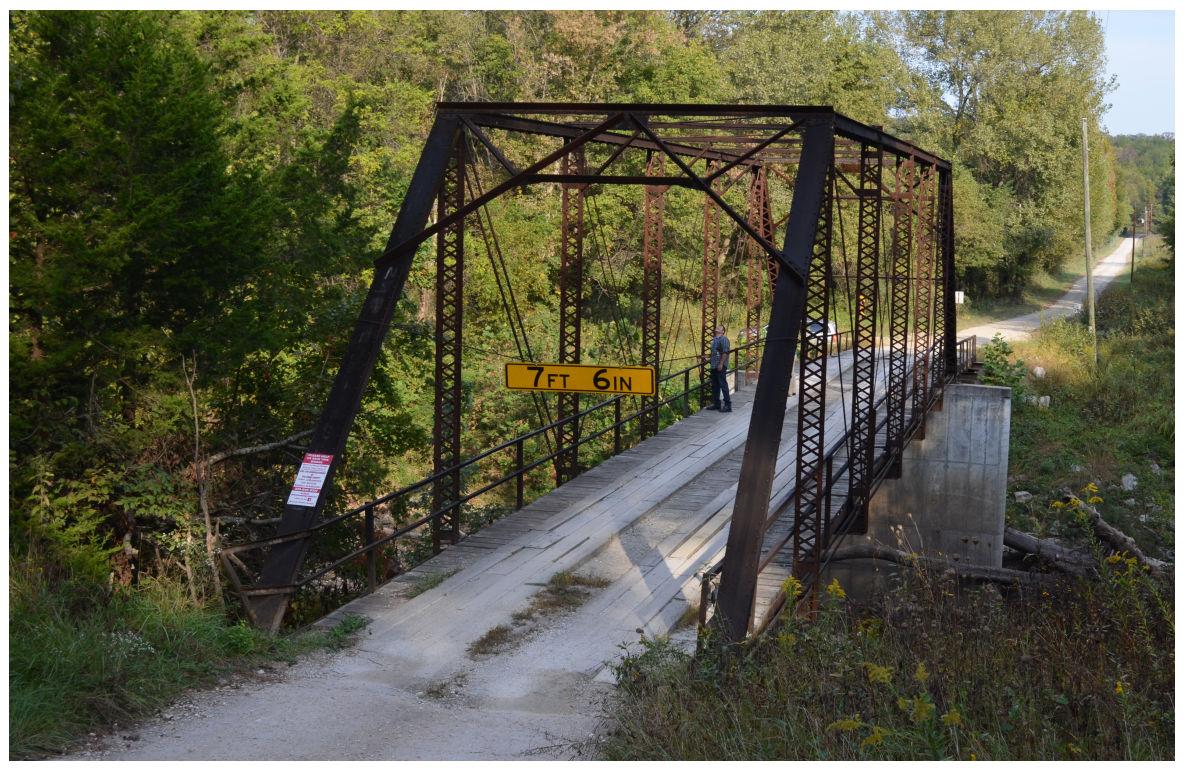

Figure 12: Fracture-critical, structurally deficient Boone County bridge.

and intervened when necessary to prevent tangling or snags throughout the testing event. For substructure inspection, the pilot and inspector relocated to the stream bank as it provided a better vantage point for visual observation of the aircraft. The tether was suspended over the side of the bridge and required constant monitoring by the crew to prevent entanglement and contact with water. Identical to the superstructure inspection, the inspector had control of the gimbal and could view captured video footage through the goggles. The pilot flew the UAV across the bottom of the deck and sides of the bridge as requested by the bridge inspector.

\section{Inspection Results \& Discussion}

The Boone County Bridge inspection was successfully completed using the developed aircraft and tethering system. The pilot was capable of maintaining the 
vehicle stable at close proximity to the bridge. Even though the truss structure presented some obstacles the pilot was able to maneuver around the complex structure and avoid contact with the structure (Figure 13). Overall, the bridge inspectors were extremely satisfied with the clarity and sharpness of the video transmitted to the goggles and ground monitor particularly, when underneath the deck. An example of the onboard recorded

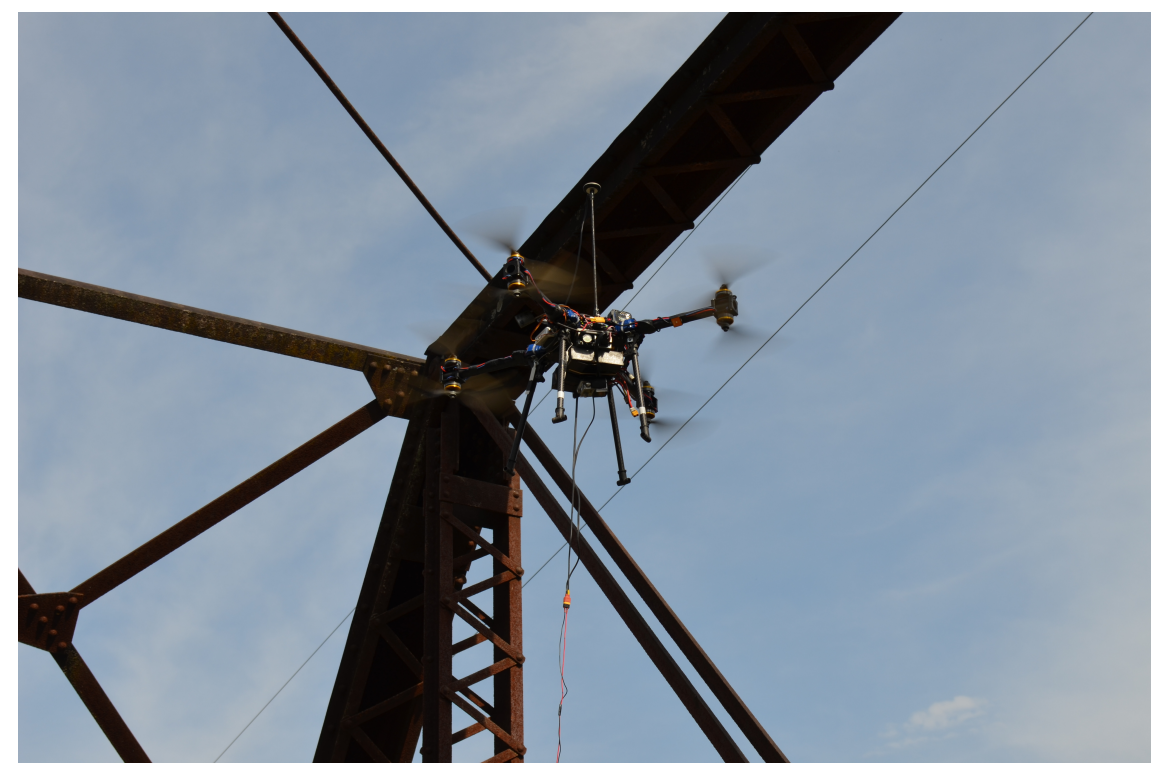

Figure 13: Tethered aircraft during superstructure inspection.

video collected during substructure inspection is shown in Figure 14. The screen capture highlights the clarity of the imagery captured by the aircraft, providing confidence in the inspector conclusions from the video data. From the collected video data, inspectors were able to determine that the substructure is in "better-than-expected" condition.

During the field testing, the inspection crew and operators provided substantial feedback. A major limitation encountered was the necessity for the pilot and inspector to be physically next to each other at all times. Often the pilot would need to maneuver for 


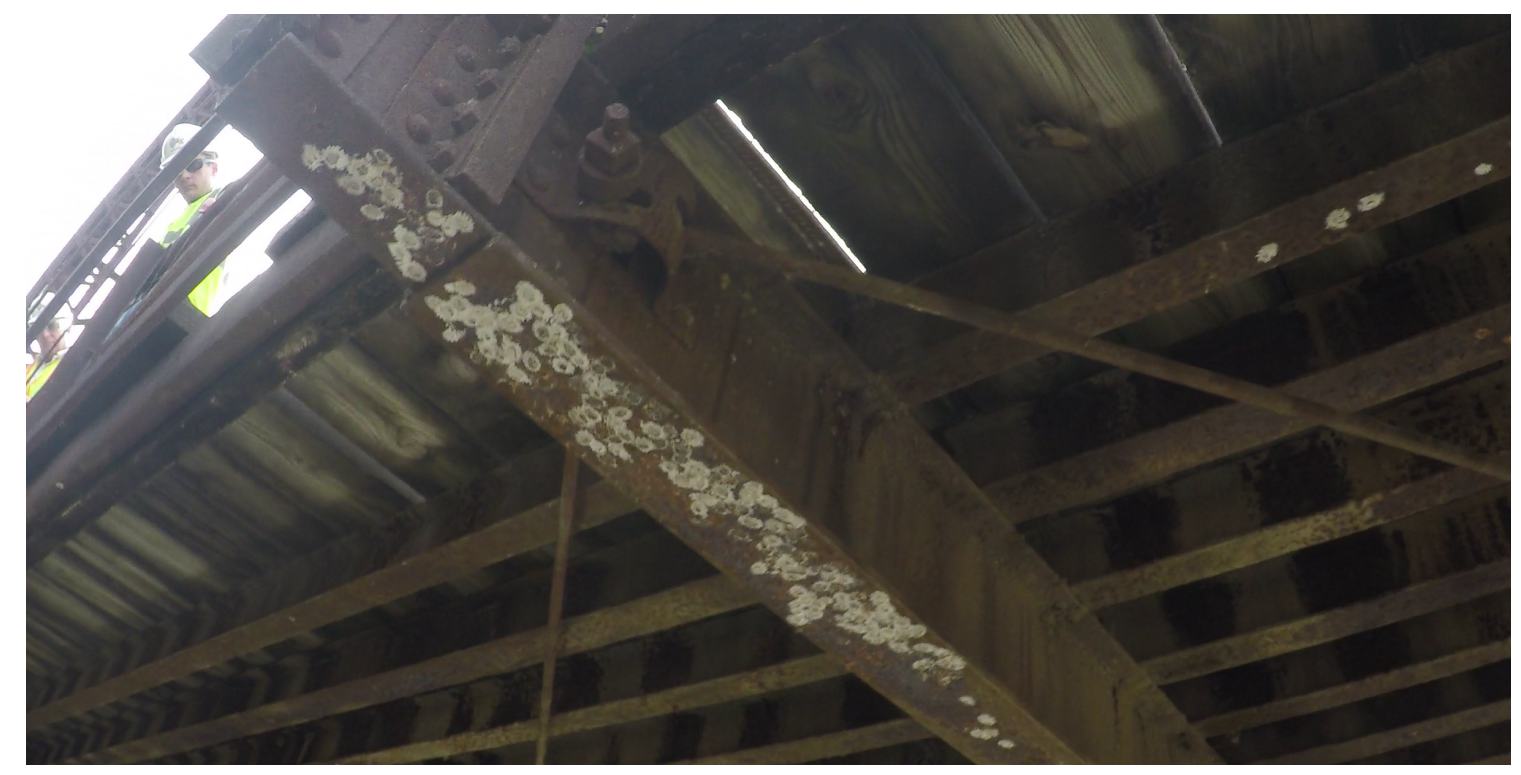

Figure 14: Camera footage from tethered aircraft during substructure inspection.

a better vantage of the aircraft, while the inspector could be primarily stationary. Another key issue was the extreme contrast in the video data when attempting to look into a heavily shaded connection. This was commonly encountered when examining the upper deck of the bridge. Without the ability to modify the camera exposure in real-time, it is quite challenging to visually capture all of the shaded and exposed connections. Overall, the inspection crew was enthusiastic about the potential for unmanned aircraft inspections and were excited to see the capabilities of the system.

\section{Conclusion}

The third generation inspection system was the first out of two previous prototypes to successfully conduct a bridge inspection. The third generation aircraft's motors and propellers produced sufficient thrust without exceeding power supply voltage and 
current limitations. The optimization method for determining appropriate tether conductor length, size, and material successfully determined appropriate tether specifications that allowed the aircraft to remain in flight for the desired amount of time. The video transmitting system provided inspectors with an efficient method of monitoring difficult-to-reach areas especially at the under deck. The inability to manually control camera exposure proved to be a drawback as heavily shaded areas were difficult to asses properly. Limitations experienced by the third generation prototype were addressed by the fourth and final generation prototype. 


\section{CHAPTER 6}

\section{FOURTH GENERATION PROTOTYPE Introduction}

The fourth generation prototype was built in response to inspector and pilot feedback following the Boone County Bridge inspection. The prototype held the same capabilities as the third generation in addition to meeting the needs as prescribed by pilot and inspector. The prototype resolved the issues of conflicting ground stations, ground monitoring, power supply inefficiencies, and video quality. This chapter details the methodology and results necessary to convert an off-the-shelf drone into a fully functioning prototype. This chapter also introduces quantitative techniques taken to measure UAS flying quality. The last section of the chapter details how quantitative results are used to identify weaknesses in the UAS.

\section{Prototype Development}

The fourth generation prototype was built in response to inspector and pilot feedback following the Boone County Bridge inspection. The prototype held the same capabilities as the third generation in addition to meeting the needs as prescribed by pilot and inspector. The prototype resolved the issues of conflicting ground stations, ground monitoring, power supply inefficiencies, and video quality. 
The base for the fourth generation prototype is an off-the-shelf DJI Inspire 1 coupled with a high voltage powered tether system (Figure 15). The UAS operating system comes prebuilt minimizing aircraft setup time. The aircraft has an empty weight (excluding battery) of $2.35 \mathrm{~kg}(5.17 \mathrm{lb})$ and is capable of generating $7.2 \mathrm{~kg}(15.8 \mathrm{lb})$ of maximum thrust. The aircraft requires approximately $1300-1400 \mathrm{~W}$ to operate at full throttle. At hover the aircraft typically draws less than $450 \mathrm{~W}$. Flight is controlled by a custom DJI flight controller with three flight modes available giving the option to use GPS for aircraft positioning. The Inspire is powered through a custom built 24VDC LiPo battery which provides approximately 15 minutes of flight time. The vehicle is also equipped with a custom camera and gimbal. The camera supports $4 \mathrm{k}$ video capture and 12 megapixel photo capture. The flight controller has an integrated DJI Lightbridge relieving inconveniences with hardware encountered with the third generation aircraft. A mobile device such as a tablet connects via USB and mounts on the controller providing the pilot control over several camera functions as well as vehicle monitoring capabilities through onscreen displays. These capabilities are supported through the DJI Pilot App. Video recorded by the onboard camera is transmitted and displayed on the mobile device in real time. Video can be saved on the tablet as well as on the camera memory card.

The flight system gives the option of operating in its Dual Remote Controllers Mode. The "Master" controller controls aircraft flight while the "Slave" controller controls gimbal movement and camera parameters. The dual controller mode no longer limits inspector and pilot mobility as experienced with the third generation aircraft. The remote control system used in the third generation required pilot and inspector to always be within 


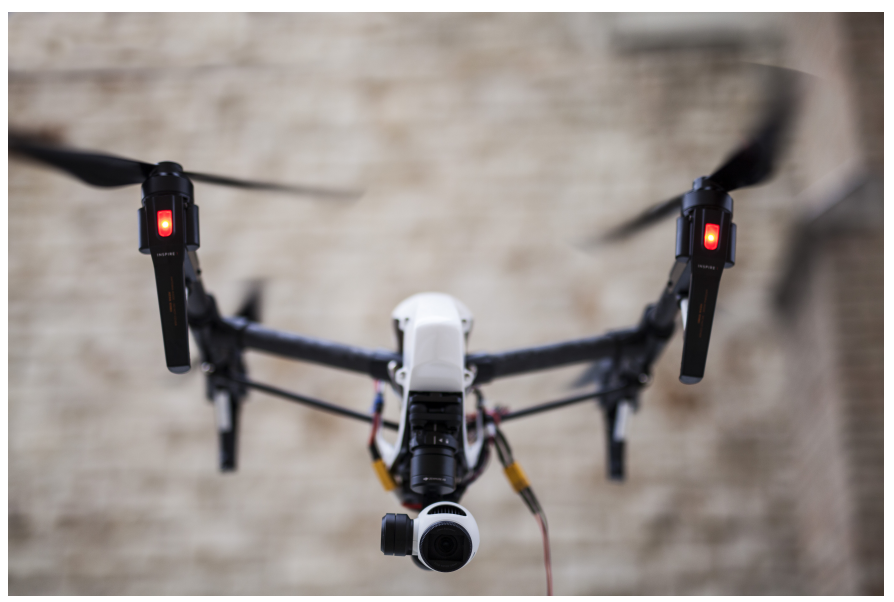

Figure 15: Commercial-off-the-shelf DJI Inspire 1 aircraft during hovering flight operations.

three feet of each other since both controllers connected to the Lightbridge. The gimbal is capable of $360^{\circ}$ rotation and $120^{\circ}$ vertical position. Through the DJI Pilot App The "Slave" controller can control camera exposure, relieving issues such as sun glare and shadows. Figure 16 shows an image captured by the aircraft camera of a steel construction with limited exposure. The construction consists of bolts, fittings, connections, rust, etc. which are also seen on bridges. The underexposure limits the inspector from clearly assessing the details of the bolts, fittings, etc. Figure 16 is juxtaposed by Figure 17 which shows a photo of the same structure taken with the aircraft camera after the camera has been adjusted for overexposure. The overexposure eliminates the shadows obstructing the inspectors view. Unlike the third generation prototype camera, the Inspire camera eliminates the trouble commonly encountered when examining the upper deck of a bridge as explained in 5. 


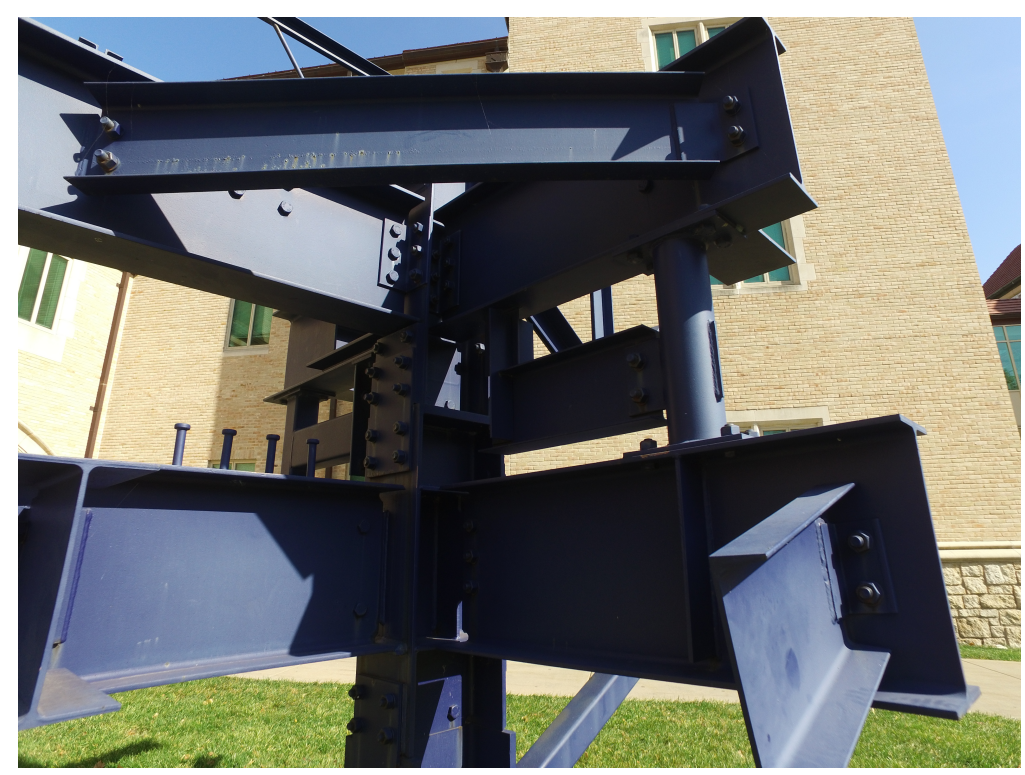

Figure 16: Steel construction with manual underexposure.

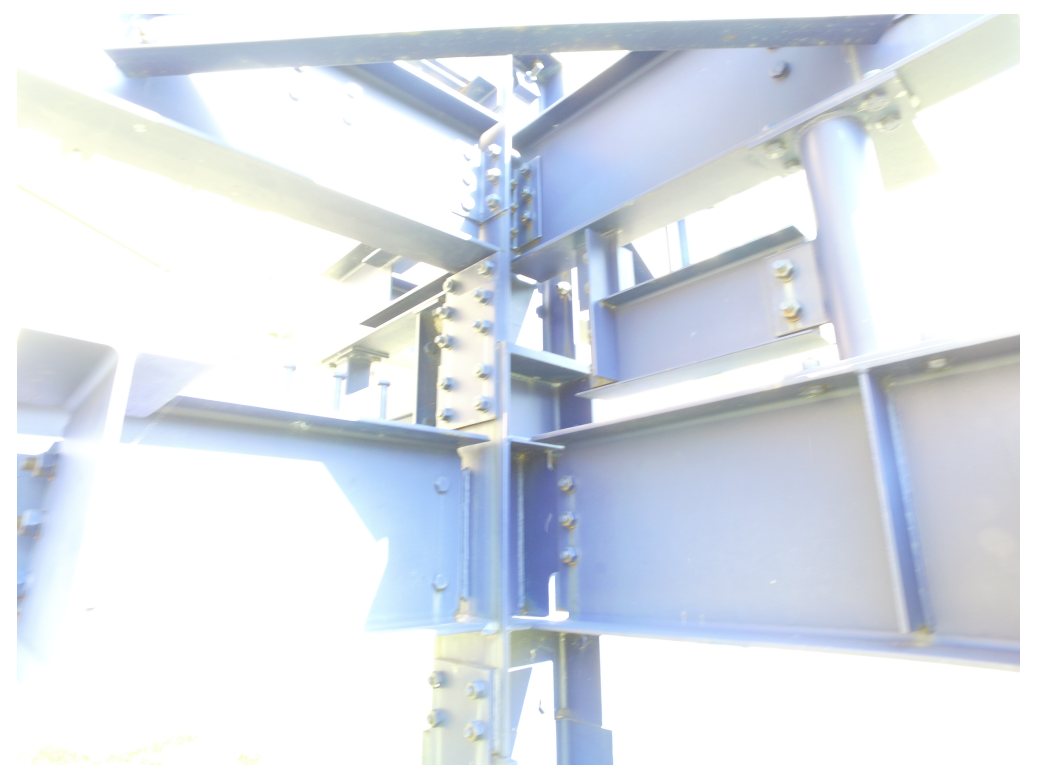

Figure 17: Steel construction with manual overexposure. 


\section{Modified Cooper-Harper rating scale for inspection based UAS flying operations.}

Due to the subjective nature of bridge inspections, there is a need for quantifying the handling qualities during an inspection. The popular Cooper-Harper piloted rating scale method was chosen as a means of evaluating the aircraft [42]. Typically, pilots quantitively rate their handling of tasks based on the scale. A modified Cooper-Harper piloted rating scale was created to measure the handling of a UAS [43]. Good handling of a UAS is important for safety and efficiency. Handling of a UAS often becomes compromised in the absence of GPS lock especially for less-experienced pilots. When inspecting the under deck of the Boone Co. bridge the UAS was unable to secure a GPS lock. The very structure being inspected became an obstacle to maintaining stability. To evaluate the handling of the DJI Inspire in the absence of GPS lock the pilot conducted mock inspections and implemented the modified Cooper-Harper rating scale. The developed Cooper-Harper scale (Figure 18) used by the pilot to asses the DJI Inspire is based heavily on Cotting's modified version of the scale. 


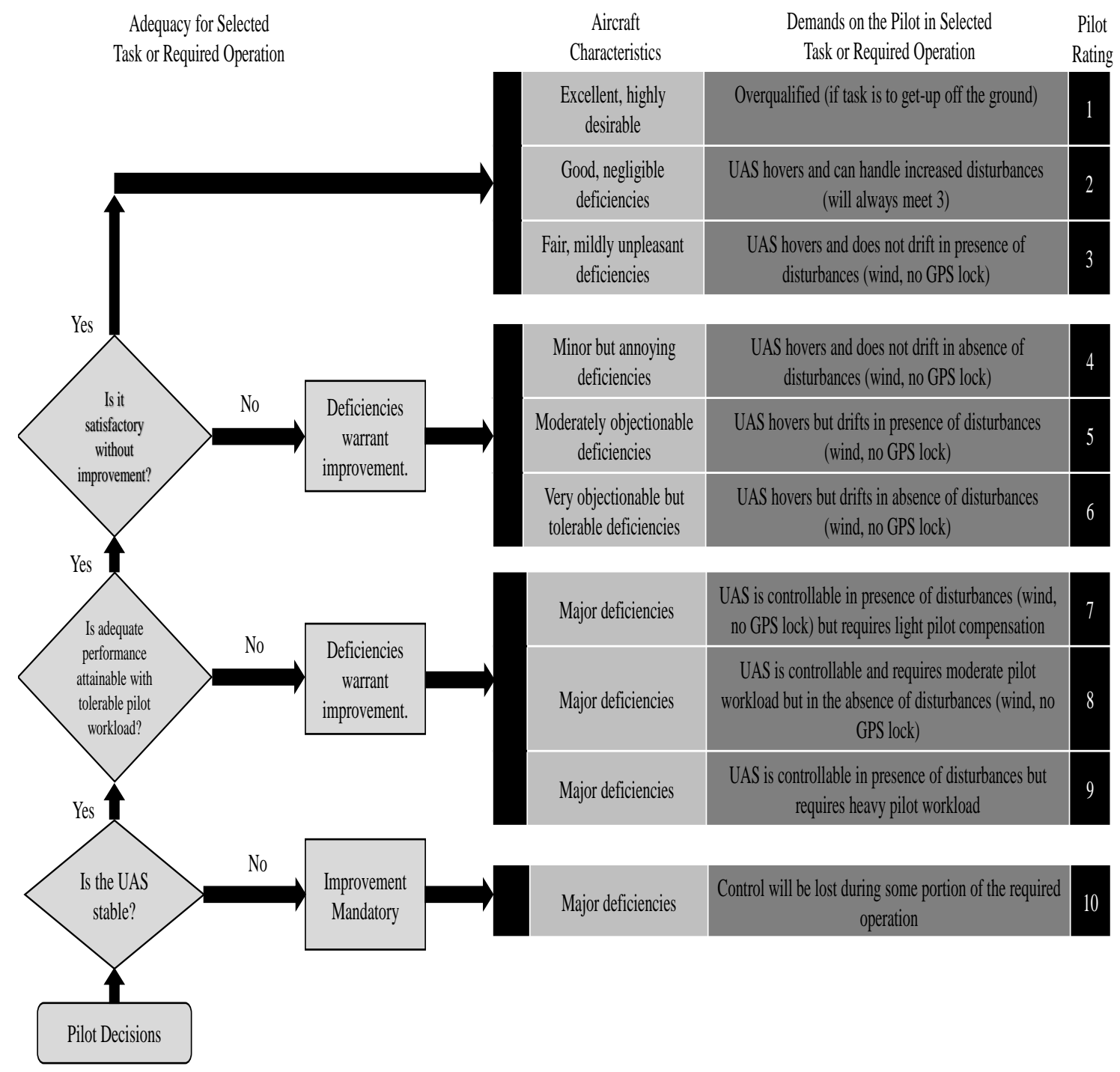

Figure 18: Modified Cooper-Harper rating scale.

To use the scale, the pilot begins at the bottom left (Pilot Decisions) of Figure 18 and follows the arrows depending on their response to the presented questions. The pilot then determines if the vehicle is stable, performs adequately with workload, or does 
not require improvements. The three categories direct the pilot to a rating scale ranging from 1 to 10 (1 signifying a highly desirable aircraft and 10 indicating a requirement for immediate improvement) thereby quantifying the handling qualities of the UAS for specifically targeted inspection operations.

\section{Flight Quality Test Methodology}

UAS positioning in relation to a structure can significantly effect flight handling. When inspecting the substructure of the Boone County Bridge the aircraft was unable to obtain a GPS lock which effected the aircraft's longitudinal and latitudinal positioning resulting in undesired shifting. Shifting is a significant concern when flying at close distances as it can lead to collisions. Vertical shifting is also undesired especially when examining in between girders since they are in close proximity to the bridge deck. Lack of GPS lock is not expected to cause undesired vertical shifting since an onboard barometer aids the aircraft in maintaining constant altitude through pressure measurements. To further understand the effects generated by surrounding structures the aircraft was flown in an environment which presented similar obstructions. The UMKC parking garage was chosen as a viable testing sight for two reasons: the garage prevents GPS lock and enables safe and accessible flight testing. In addition to preventing GPS lock, aircraft thrust against the garage walls creates turbulence which can significantly upset flight behavior. As the aircraft nears an obstruction more turbulence is produced. The effects of turbulence create conflict between the pilot and the inspector. Where an inspector requires close range to adequately monitor structural quality, a pilot will require enough distance 
to avoid collision and turbulence effects. UAS based bridge inspections is a new concept and lacks structural guidelines for both pilots and inspectors to follow to safely and efficiently conduct bridge inspections. Developing a set of guidelines requires rigorous testing and collaboration between pilots and inspectors. To begin developing guidelines this thesis presents a revolutionary quantification of flight quality. Prior to conducting additional bridge inspections, the effects of close proximity flight need to be quantified. The pilot needs to quantify the effects of flying at different distances under strict conditions. The distance between the aircraft and surrounding obstructions can be deduced by taking into account perspective; closer objects appear larger then objects further away. Testing consisted of flying parallel to the parking structure wall while maintaining a constant distance of 1.5 and $3 \mathrm{~m}$. To determine the distance between the aircraft and the wall a 28 $\mathrm{cm}$ wide strip was placed on the wall. The strip was made by laying down two strands of tape $28 \mathrm{~cm}$ apart both vertically and horizontally along the wall. An example image from flight testing for the vertical and horizontal stability tests is shown in Figure 19 and Figure 20,respectively. Video was taken during flight testing and examined through post processing to determine the pixel width of the strip throughout the flight. A pixel width baseline was established in order to identify aircraft deviation. Video processing software measured the $28 \mathrm{~cm}$ strip as 167 and 84 pixels at 1.5 and $3 \mathrm{~m}$, respectively; any deviation from the baseline signified the aircraft was either approaching or moving away from the strip. When examining bridge girders the aircraft needs to fly horizontally while maintaining a constant vertical position (altitude) in order to avoid hitting the bridge deck; alternatively, when examining vertical steel supports on the bridge superstructure 
the aircraft needs to fly vertically while maintaining a constant horizontal position in order to avoid hitting neighboring steel supports. To assess the aircraft's ability to maintain constant vertical and horizontal positioning, the strip's midpoint relative to the camera's midpoint was measured. The video processing software arranges pixels in an x-y grid as shown in Figure 21. During horizontal flight the strip's $y$ midpoint coordinates are expected to remain fairly constant. During vertical flight the strip's $x$ midpoint coordinates are expected to remain relatively constant.

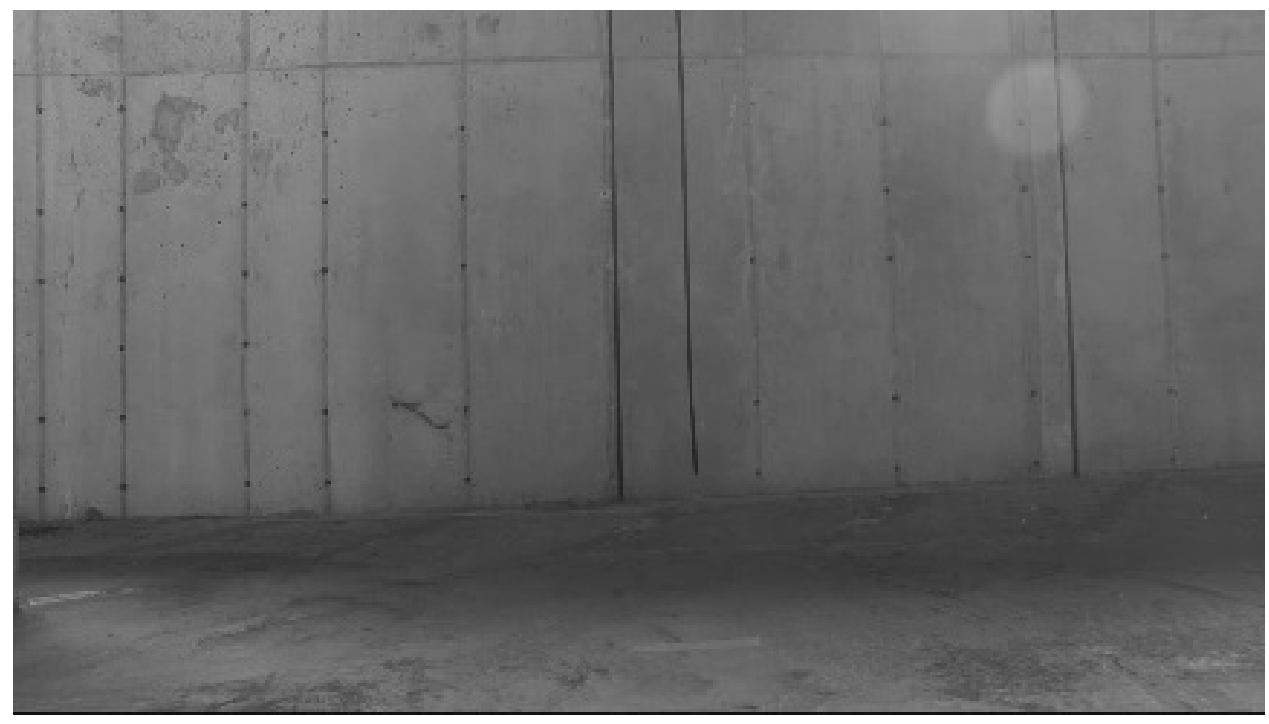

Figure 19: Vertical $28 \mathrm{~cm}$ strip used for flight quality testing. 


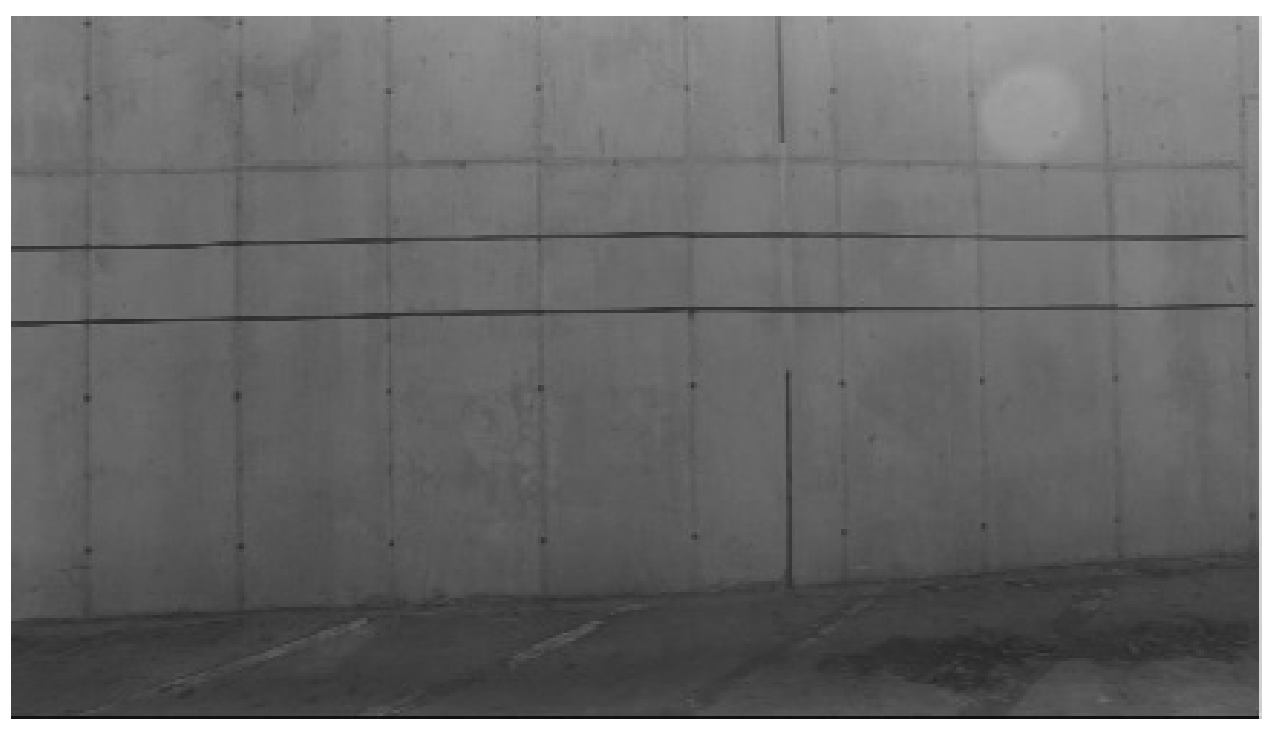

Figure 20: Horizontal $28 \mathrm{~cm}$ strip used for flight quality testing.

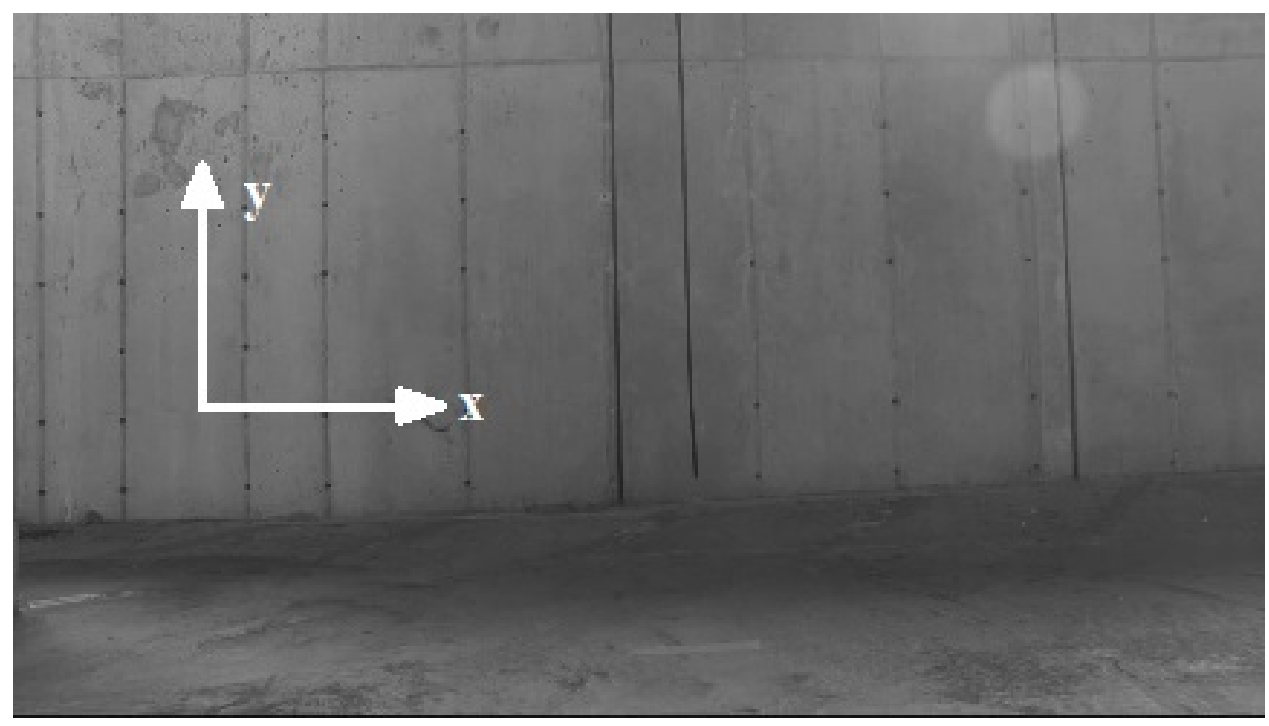

Figure 21: Horizontal $28 \mathrm{~cm}$ strip used for flight quality testing.

It was left to the pilot to maintain a constant distance from the strip as the testing was conducted. Post processing was used to identify strip pixel width deviation from the 
baseline. The pilots ability to maintain a steady distance from the established line was used as an indication of the aircraft's "controllability". The pilot conducted three flight tests at two distances $(1.5$ and $3 \mathrm{~m})$ for both the vertical and horizontal strip. Quantification of vehicle flight quality was accomplished with the modified Cooper-Harper rating scale.

\section{Post-Flight Processing of Data}

Post-flight image processing was completed using LabVIEW's Vision Assistant Virtual Instrument (VI). The VI was used to process video taken during Cooper-Harper scale testing. Video data was converted to grayscale permitting distinction between the strands of electric tap and the surrounding wall. The Vision Assistant (Figure 22) was used to calculate the strip's centroid relative to the camera's 960 x 650 pixel screen. The Vision Assistant was also used to monitor strip pixel width. Any deviation from the calibrated line was indicative of deviation from the predetermined distance set forth prior to flight testing and could indicate a lack of safe flight stability. 


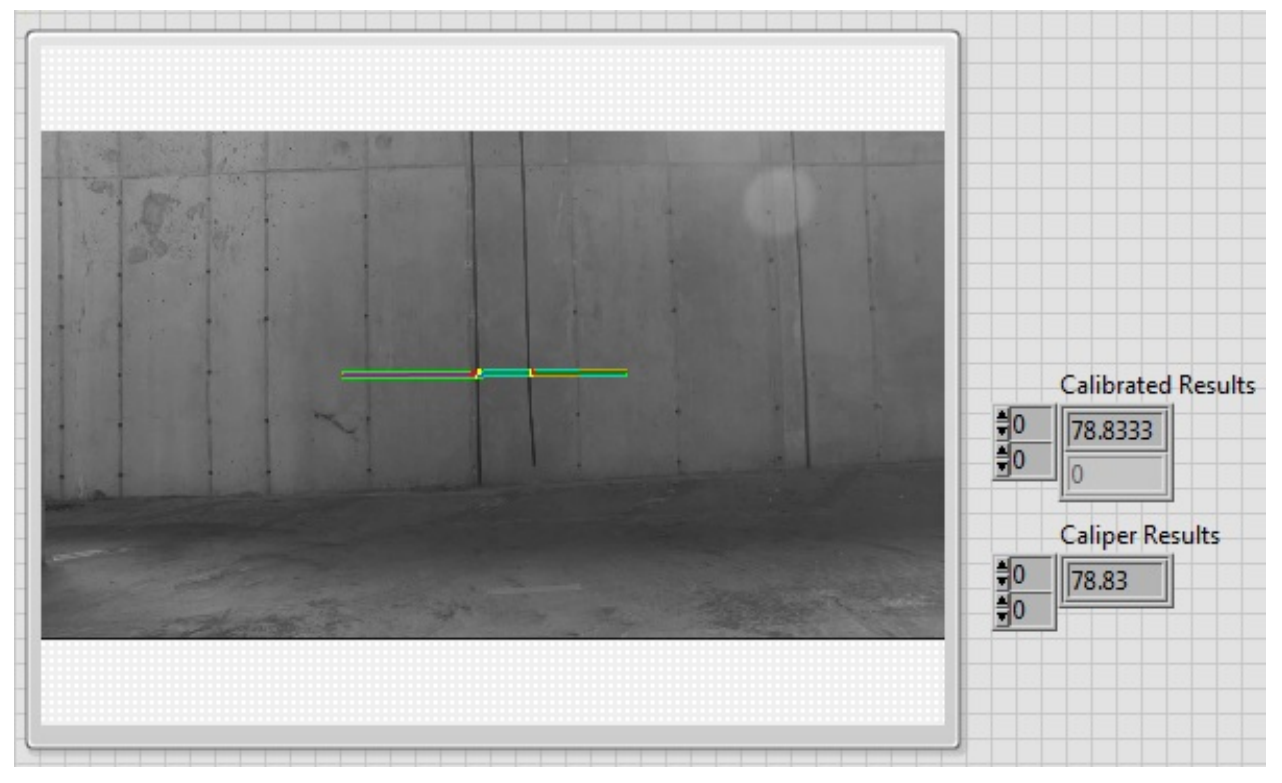

Figure 22: LabVIEW Vision Assistant screen shot.

\section{Flight Testing Results \& Discussion}

A total of 12 flight tests were conducted in the UMKC parking structure. For a baseline, the strip widths in pixels at the two flight distances are shown in Table 7. Tables 8 and 9 show the Cooper-Harper rating assigned by the pilot when completing the horizontal and vertical flight tests at each of the two distances, respectively. Flights at $3 \mathrm{~m}$ mostly received Cooper-Harper ratings of 3 . Flights at $1.5 \mathrm{~m}$ mostly received ratings of 5 indicating that there was a higher pilot workload at $1.5 \mathrm{~m}$ compared to $3 \mathrm{~m}$. Qualitatively, the aircraft itself was controllable, but due to disturbances (such as lack of GPS lock and wind kick-back), the aircraft would experience undesired drift. Drift is mostly noticeable when flying at $1.5 \mathrm{~m}$ as seen in Figure 23. 
Table 7: Strip widths

\begin{tabular}{c|c}
\hline Strip Distance $(\mathrm{m})$ & Line Width (pixels) \\
\hline 1.5 & 167 \\
\hline 3 & 84 \\
\hline
\end{tabular}

Table 8: Horizontal flight Cooper-Harper rating

\begin{tabular}{c|c|c|c}
\hline Flight Distance $(\mathrm{m})$ & Trial 1 & Trial 2 & Trial 3 \\
\hline 1.5 & 5 & 5 & 3 \\
3 & 3 & 3 & 3 \\
\hline
\end{tabular}

Table 9: Vertical flight Cooper-Harper rating

\begin{tabular}{c|c|c|c}
\hline Flight Distance $(\mathrm{m})$ & Trial 1 & Trial 2 & Trial 3 \\
\hline 1.5 & 5 & 5 & 5 \\
3 & 5 & 3 & 3 \\
\hline
\end{tabular}

Figures 23 and 24 are representative results of the pixel data from the first trials of the vertical flight test at a distance of $1.5 \mathrm{~m}$ and $3 \mathrm{~m}$, respectively. In Figure 23 the red line indicates the pixel width of a $28 \mathrm{~cm}$ strip at $1.5 \mathrm{~m}$. In Figure 24 the red line indicates the pixel width of a $28 \mathrm{~cm}$ strip at $3 \mathrm{~m}$. Figure 23 indicates that for a major duration of the flight the aircraft was further away than $1.5 \mathrm{~m}$. There is a strong contrast between Figure 23 and Figure 24 in that the pilot was able to maintain the farther intended distance of $3 \mathrm{~m}$. Both the Cooper-Harper ratings and pixel width deviation results serve as evidence supporting the intuition that closer distances are harder to maintain during flight due to the presence of obstructions while with an absence of GPS lock. Tables 10 and 11 show the average strip width and standard deviation for each trial at each of the two distances. 
For vertical flight, mean pixel widths differed by $10.1,3.0$, and 15.6 percent from the $1.5 \mathrm{~m}$ baseline for trials 1, 2, and 3, respectively. Based on Table 10 for vertical flight, mean pixel widths differed by $0.4,5.8$, and 0 percent from the 3.0 baseline for trials 1 , 2, and 3, respectively. For horizontal flight, mean pixel widths differed by 8.6, 11.1, and 8.3 percent from the $1.5 \mathrm{~m}$ baseline for trials 1, 2, and 3, respectively. For horizontal flight, mean pixel widths differed by $1.0,1.1$, and 20.5 percent from the 3.0 baseline for trials 1,2 , and 3 , respectively. Table 10 shows that on average the aircraft deviated significantly more from the intended distance for $1.5 \mathrm{~m}$ flights than for $3.0 \mathrm{~m}$ flights. It is not appropriate to compare standard deviations between the two flight distances since they encompass different ranges; instead, the standard deviations are treated as percentages of their respective mean. For vertical flight at $1.5 \mathrm{~m}$, standard deviations account for 7.3, 11.6, and 8.1 percent of their respective mean for trials 1,2 , and 3 while for vertical flights at $3 \mathrm{~m}$, standard deviations account for 3.7, 5.5, and 2.2 percent of their respective mean for trials 1, 2, and 3. For horizontal flight at $1.5 \mathrm{~m}$, standard deviations account for 6.5, 3.4, and 27.4 percent of their respective mean for trials 1,2 , and 3 while for horizontal flights at $3 \mathrm{~m}$, standard deviations account for 2.1,3.2, and 43.6 percent of their respective mean for trials 1, 2, and 3. Higher standard deviations among $1.5 \mathrm{~m}$ flights are indicative of the greater differences between pixel width measurements signifying that the aircraft did not maintain a steadier distance throughout flight. Standard deviations are lower for 3.0 m flights indicating that pixel width measurements had a greater consistency throughout flight signifying steadier flight. There are inconsistencies and "jumps" throughout pixel width data as highlighted in Figure 25. The jumps are attributed to the Vision Assistant 
software unable to distinguish the two strips of tape in the flight video. Due to differences in lighting, wall imperfections such as dirt and debris as highlighted in Figure 26, the Vision Assistant had trouble properly identifying the strands of tape used to make the $28 \mathrm{~cm}$ strip. As a result, the Vision Assistant would over-measure the strip pixel width. Adjustments were made through the software to not pickup inconsistencies and although not all imperfections could be eliminated the most accurate configuration was achieved. All flight pixel width data was adjusted in order to eliminate the occasional "jumps" and converted from pixels to distance (m) using the baseline measurements from Table 7 and are presented in Appendix A. As can be seen in Figure 25 the inconsistencies follow the same pattern as the surrounding data and experiences similar shifts. Adjustments were made by either increasing or decreasing the data "jumps" along the pixel width axis so as to align it with the rest of the data. The adjusted data was used in calculations of mean and standard deviation. Data was converted from pixel width to distance in order to provide ease of visualization as to how far the aircraft was drifting from its intended target. 


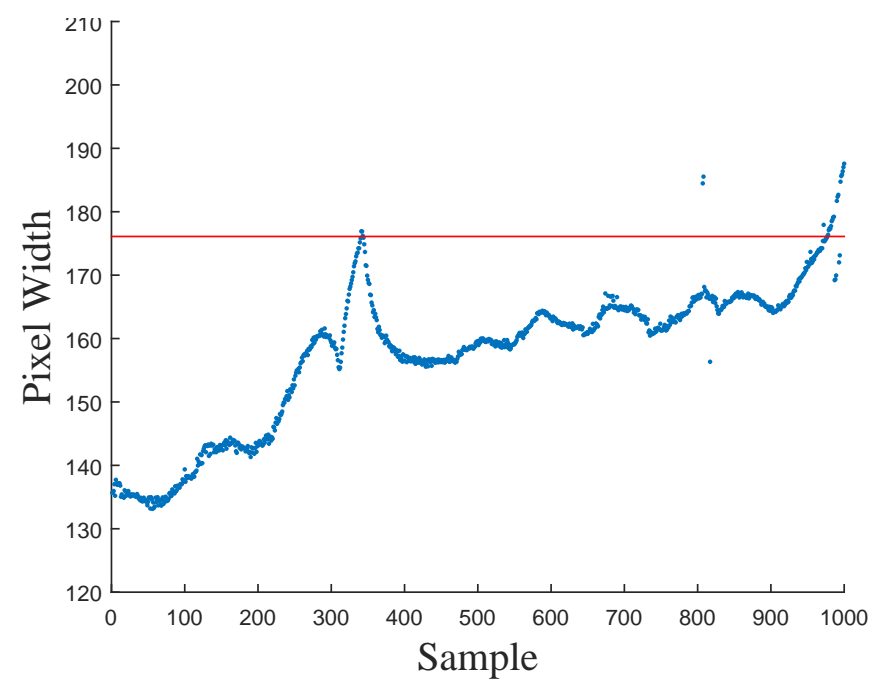

Figure 23: Vertical strip pixel width at $1.5 \mathrm{~m}$ : Trial 1.

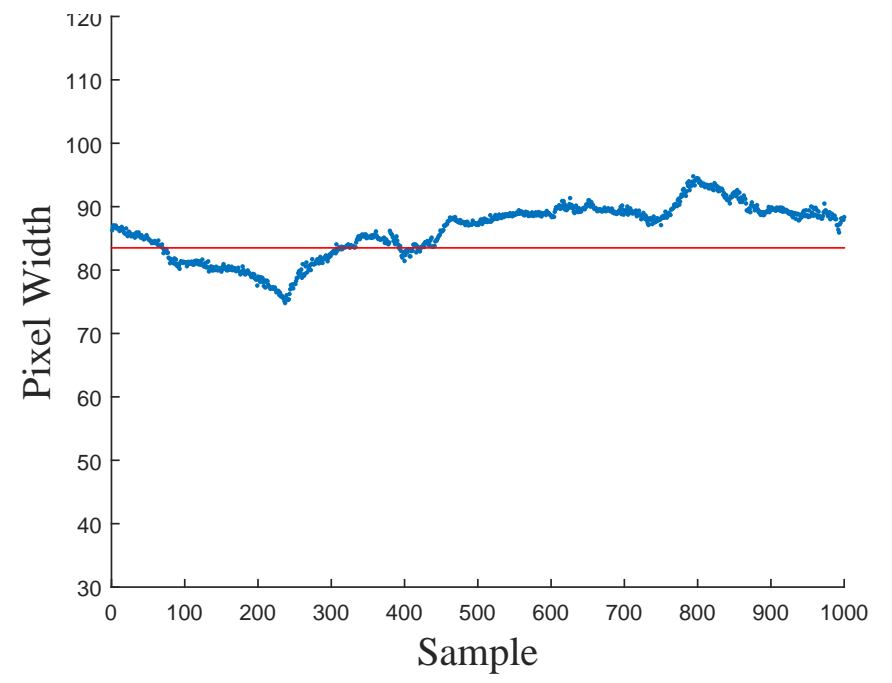

Figure 24: Vertical strip pixel width at $3.0 \mathrm{~m}$ : Trial 1. 
Table 10: Vertical-flight line pixel widths

\begin{tabular}{c|cc|cc|cc}
\hline Distance $(\mathrm{m})$ & \multicolumn{2}{|c|}{ Trial 1 } & \multicolumn{2}{c|}{ Trial 2 } & \multicolumn{2}{c}{ Trial 3 } \\
\hline \multirow{3}{*}{1.5} & Mean & SD & Mean & SD & Mean & SD \\
\cline { 2 - 7 } 3.0 & 150.1 & 11.0 & 172.0 & 19.9 & 141.1 & 11.4 \\
\hline
\end{tabular}

Table 11: Horizontal-flight line pixel widths

\begin{tabular}{c|cc|cc|cc}
\hline Distance $(\mathrm{m})$ & \multicolumn{2}{|c|}{ Trial 1 } & \multicolumn{2}{c|}{ Trial 2 } & \multicolumn{2}{c}{ Trial 3 } \\
\hline \multirow{3}{*}{1.5} & Mean & SD & Mean & SD & Mean & SD \\
\cline { 2 - 7 } 3.0 & 152.7 & 10.0 & 148.4 & 5.0 & 153.2 & 42.0 \\
& 82.2 & 1.7 & 82.1 & 2.6 & 66.0 & 28.8 \\
\hline
\end{tabular}

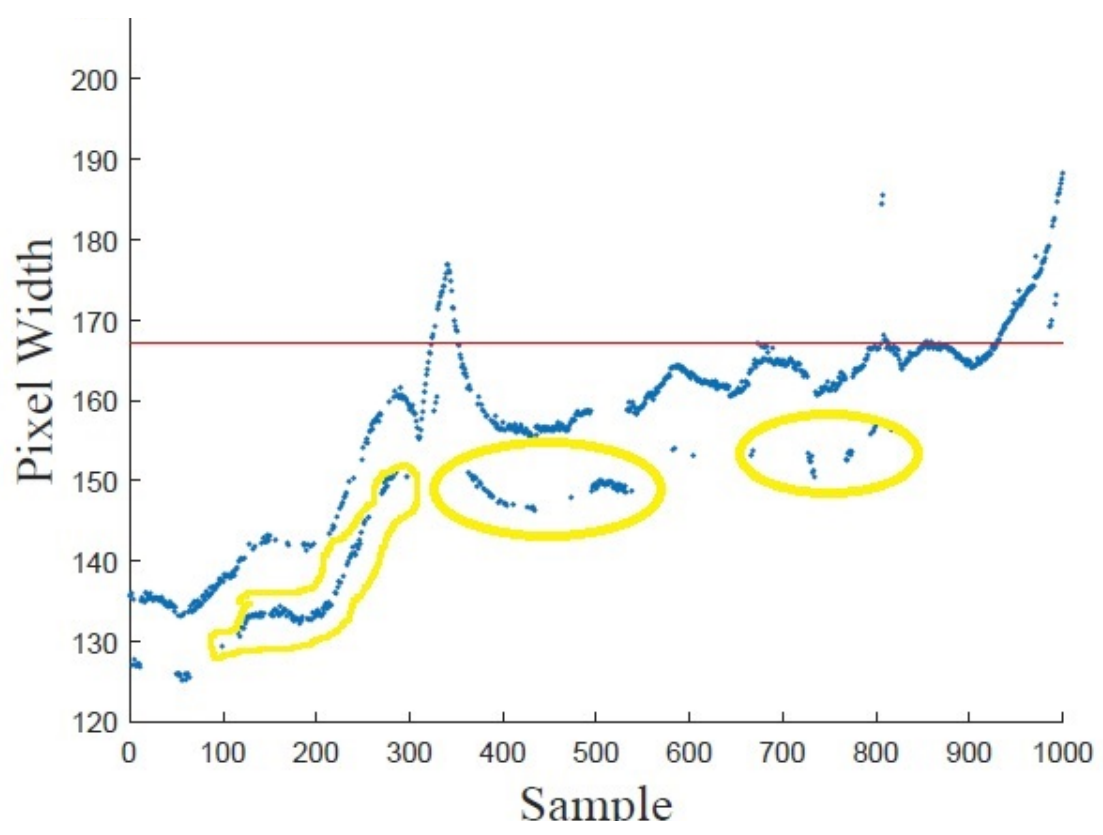

Figure 25: Pixel width data inconsistencies. 


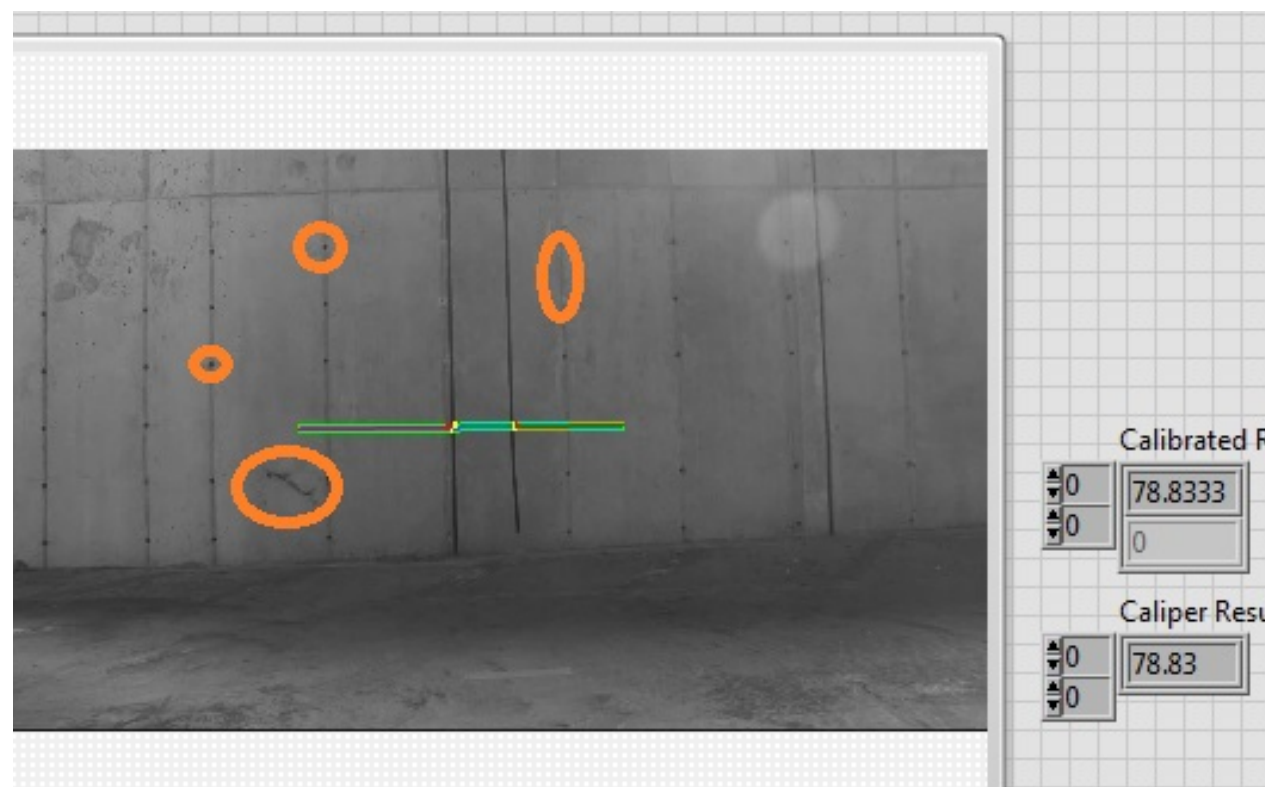

Figure 26: Parking structure wall imperfections.

Figures 27 and 28 are representative results of the mid point deviation pixel data of the vertical flight test at a distance of $1.5 \mathrm{~m}$ and $3 \mathrm{~m}$, respectively. In each plot a red marker is used to indicate the average pixel $y$ coordinate. As with Figures 23 and 24 there is a noticeable deviation away from the average pixel coordinate when flying at a distance of $1.5 \mathrm{~m}$. Figures 27 and 28 further support the intuitive notion that maintaining steady flight is is harder to achieve at closer flying distances. Tables 12 and 13 show the average mid point deviation and standard deviation for each trail at each of the two distances. The pilot was able to complete the task although with significant deviations when flying at $1.5 \mathrm{~m}$. There exists a stark difference between standard deviations for horizontal and vertical flight regardless of flight distance; standard deviations are higher for vertical flight signifying that the aircraft encountered significant sideways motion as it descended and 
ascended. When flying horizontally the aircraft was able to sustain a more steady vertical distance due to the aide of the onboard barometer which measured altitude. As with the pixel width data, there are inconsistencies and "jumps" throughout the midpoint pixel data. All flight pixel width data was adjusted in order to eliminate the occasional "jumps" and converted from pixels to distance $(\mathrm{m})$ using the baseline measurements from Table 7 and are presented in Appendix B. Maintaining the aircraft at the desired distance of $1.5 \mathrm{~m}$ required significant effort from the pilot as indicated by the higher Cooper-Harper rating (Tables 8, 9). As expected, pilot workload increases as the aircraft decreased its distance from the wall. Flight tests support intuition but are only preliminary. Results attained from Cooper-Harper ratings and post processing of flight video indicate that greater pilot workload is necessary when flying at distances of $1.5 \mathrm{~m}$ as opposed to when flying at 3 m. Although it is more difficult to maintain steady flight at $1.5 \mathrm{~m}$, the difficulty experienced by the pilot may not be experienced by the inspector. Perhaps unsteady lateral and longitudinal motion at $1.5 \mathrm{~m}$ is not of great concern when assessing bridge components and $1.5 \mathrm{~m}$ is an appropriate flight distance. The aforementioned questions cannot be addressed until further testing is performed both to assess pilot and inspector workload. Another limitation exists in that all flight tests were conducted by one pilot with approximately 72 hours of flight experience with the specific aircraft used for testing. Although Cooper-Harper rating scales are constructed with the aim of establishing objective guidelines there is still room for pilots of varying experience to assign different ratings. More accurate Cooper-Harper ratings can be obtained by performing tests with a wide variety of experienced pilots. 


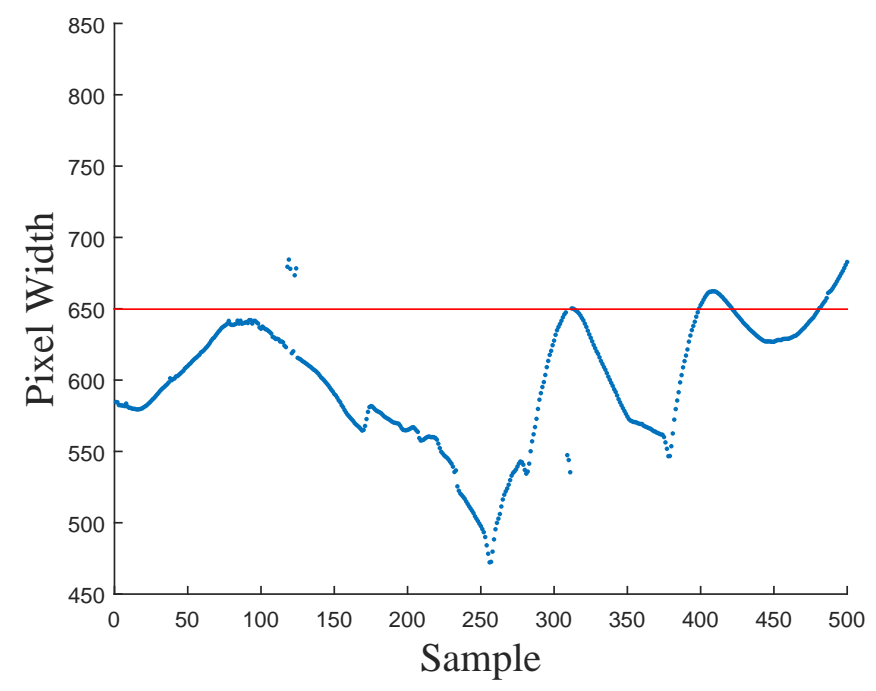

Figure 27: Vertical strip midpoint deviation at $1.5 \mathrm{~m}$ : Trial 1.

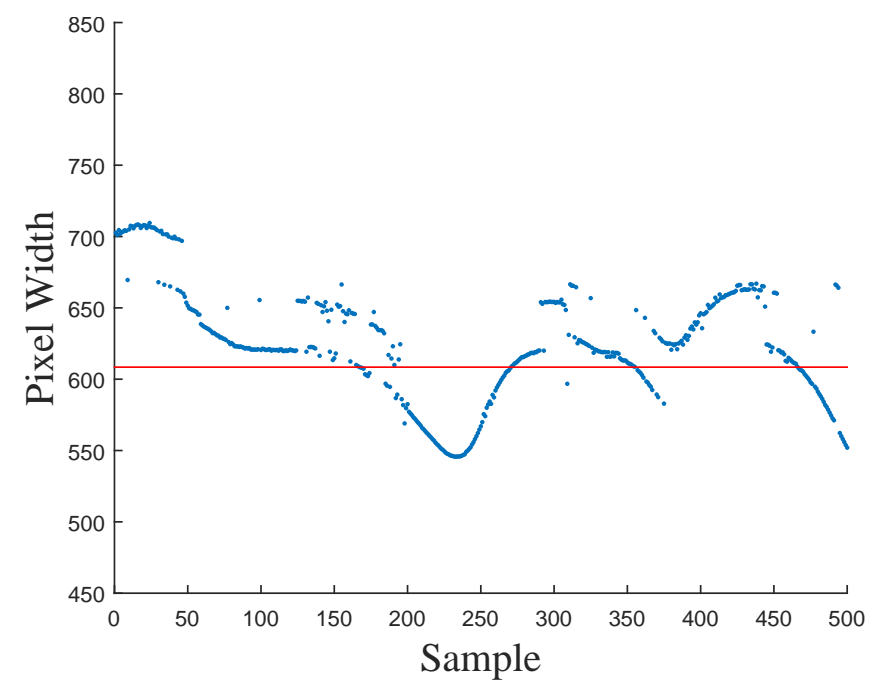

Figure 28: Vertical strip midpoint deviation at $3.0 \mathrm{~m}$ : Trial 1. 
Table 12: Horizontal flight marker mid point

\begin{tabular}{c|cc|cc|cc}
\hline Distance $(\mathrm{m})$ & \multicolumn{2}{|c|}{ Trial 1 } & \multicolumn{2}{c|}{ Trial 2 } & \multicolumn{2}{c}{ Trial 3 } \\
\hline \multirow{3}{*}{1.5} & Mean & SD & Mean & SD & Mean & SD \\
\cline { 2 - 7 } 3.0 & 264.1 & 26.9 & 255.6 & 19.2 & 250.1 & 35.7 \\
& 263.2 & 6.6 & 286.8 & 27.6 & 252.5 & 12.6 \\
\hline
\end{tabular}

Table 13: Vertical flight marker mid point

\begin{tabular}{c|cc|cc|cc}
\hline Distance $(\mathrm{m})$ & \multicolumn{2}{|c|}{ Trial 1 } & \multicolumn{2}{c|}{ Trial 2 } & \multicolumn{2}{c}{ Trial 3 } \\
\hline \multirow{3}{*}{1.5} & Mean & SD & Mean & SD & Mean & SD \\
\cline { 2 - 7 } 3.0 & 649.7 & 81.6 & 630.4 & 64.4 & 621.1 & 64.8 \\
& 632.0 & 59.7 & 615.8 & 50.1 & 565.3 & 25.8 \\
\hline
\end{tabular}

\section{Conclusion}

The fourth generation aircraft overcame the limitations presented by the third generation system. The fourth system gives the inspector the ability to manually control camera exposure. In addition, pilot and inspector can now operate the aircraft and camera/gimbal, respectively, wirelessly. Most importantly, a method of quantifying the aircraft's handling was developed and implemented. There is currently no set of guidelines for UAS based bridge inspections for operating an aircraft in a GPS denied environment when in close proximity to a structure. By quantifying UAS handling in strict scenarios this chapter marks the beginning of the development of guidelines for choosing appropriate flight distances. Results show that attempting to fly at $1.5 \mathrm{~m}$ requires a high amount of pilot input to sustain a constant distance. The pilot workload decreases significantly when attempting to maintain a distance of $3 \mathrm{~m}$. Inspector input should also be considered 
if an appropriate flight distance is to be determined. Workload is expected to decrease as distance decreases; however, inspector workload will increase. A viable median needs to be identified at which both inspector and pilot are satisfied. 


\section{CHAPTER 7}

\section{CONCLUSION}

Four bridge inspection UAS prototypes were designed where each one built upon the successes of the previous and resolved the issues that prevented it from performing the desired task. The first generation introduced the AC-to-DC high power converter that replaced the onboard LiPo battery. The power supply was slightly altered and used to power the first generation aircraft. Based on preliminary test results the aircraft could not function properly given the set of motors, propellers, and power supply being used leading to power supply failure. To generate sufficient lift the motors would require a much greater amount of power given the length of tether being used. A tether of any significant length experienced voltage drop. This lead to a new prototype that could operate within acceptable power limitations set forth by the power supply being used. A new set of motors were need to be capable of generating more lift with less current than that required by the first generation motors. A new set of motors called for a new UAS platform that could handle its potential thrust. As a result, a different UAS platform was constructed. Hardware configuration was determined using ANOVA testing. The ANOVA testing indicated that the previously used propellers and power supply would suffice and provide the aircraft with sufficient power and trust. The second generation prototype had difficulties flying stably calling for the need of a third prototype.

The third prototype witnessed an actual bridge inspection allowing the pilot and 
prototype designer to use feedback in addressing inspection system shortcomings. A fourth generation prototype was constructed that addresses the issues encountered by the three previous systems. Through development of the fourth generation inspection aircraft a new metric was established to measure UAS handling. Previously, UAS were used as an exploratory alternative without official use in the area of bridge inspections. Aircraft inspection results using the third generation system were officially used in assessing the state of the Boone County bridge. Few metrics exist for categorizing unmanned aircraft; the work presented in this thesis marks the first steps taken to quantitatively measure UAS stability in GPS denied environments when in close proximity to concrete-steel structures. The stability of other platforms can now be quantified using the proposed method described in this thesis.

Unmanned aerial vehicles continue to play an important role in research and civilian applications. A tethered power system was developed rendering unlimited flight times. The system's maneuverability and image and video transmission is clear and highly versatile that it serves as a strong substitute for direct human inspection. Although the current inspection aircraft developed herein cannot entirely replace the human factor in bridge inspections it has proven to utilized as a strong and resourceful tool. 


\section{APPENDIX A}

This appendix presents all flight pixel width data for vertical and horizontal flight tests at distances of 1.5 and 3 meters. Data is adjusted in order to eliminate the occasional "jumps" and inconsistencies and converted from pixels to distance (m) using the baseline measurements from Table 7 and are presented here. Adjustments were made by either increasing or decreasing the data "jumps" along the pixel width axis so as to align it with the rest of the data. Data was converted from pixel width to distance in order to provide ease of visualization as to how far the aircraft was drifting from its intended target. Red lines indicate the respective target flight distance.

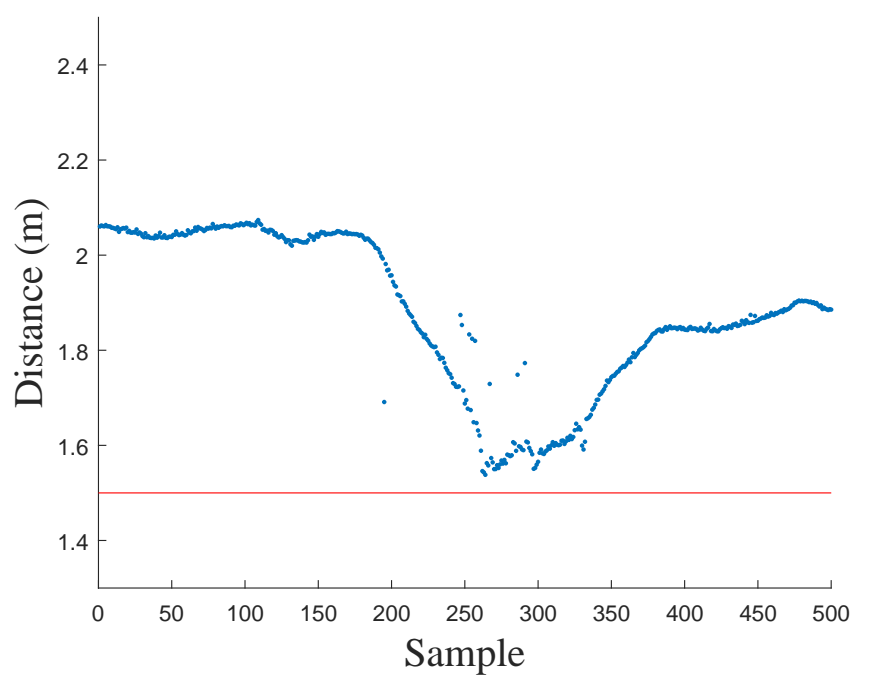

Figure A.1: Horizontal deviation at $1.5 \mathrm{~m}$ : Trial 1. 


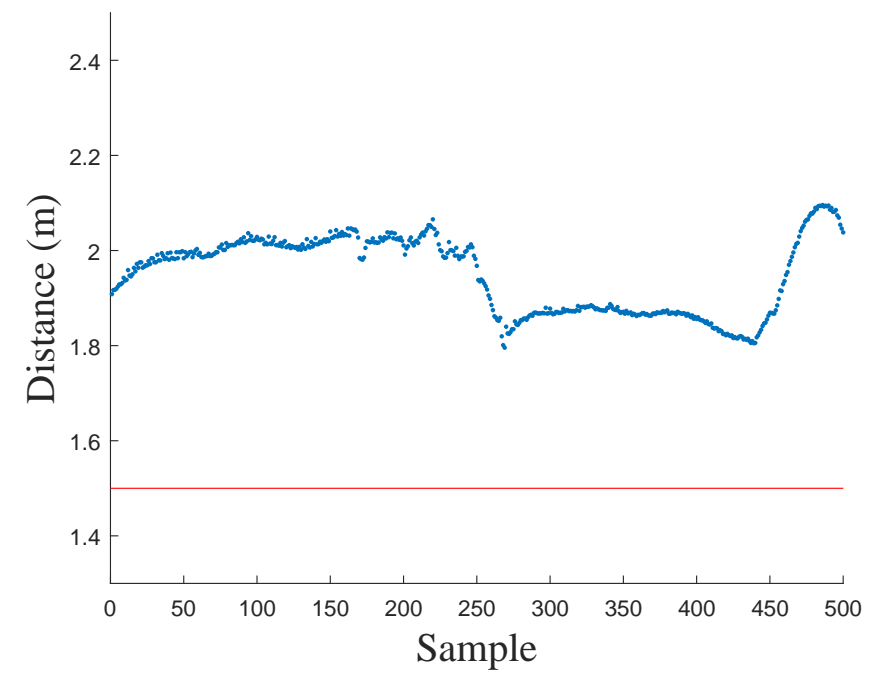

Figure A.2: Horizontal deviation at $1.5 \mathrm{~m}$ : Trial 2.

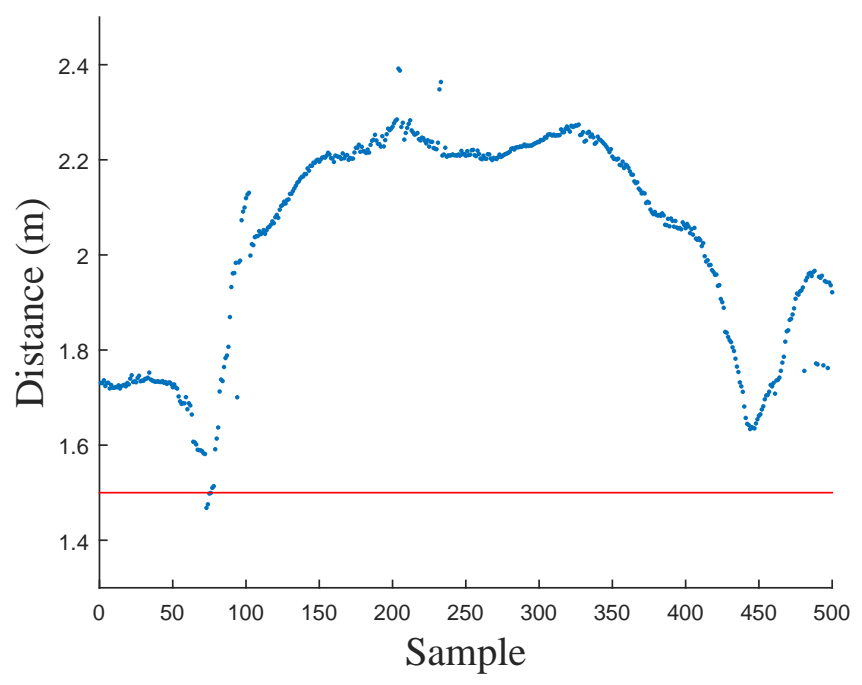

Figure A.3: Horizontal deviation at $1.5 \mathrm{~m}$ : Trial 3. 


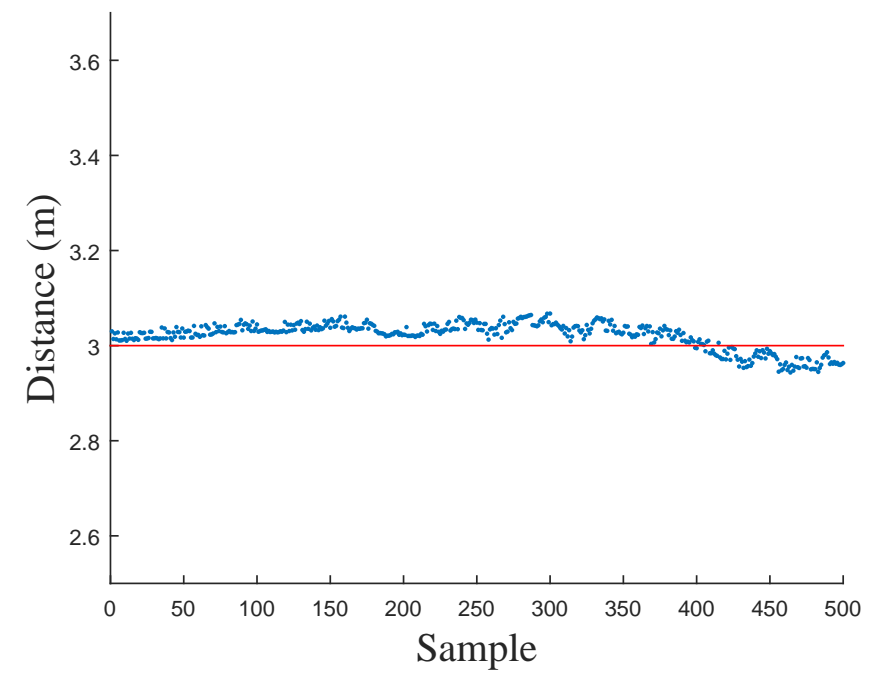

Figure A.4: Horizontal deviation at $3.0 \mathrm{~m}$ : Trial 1.

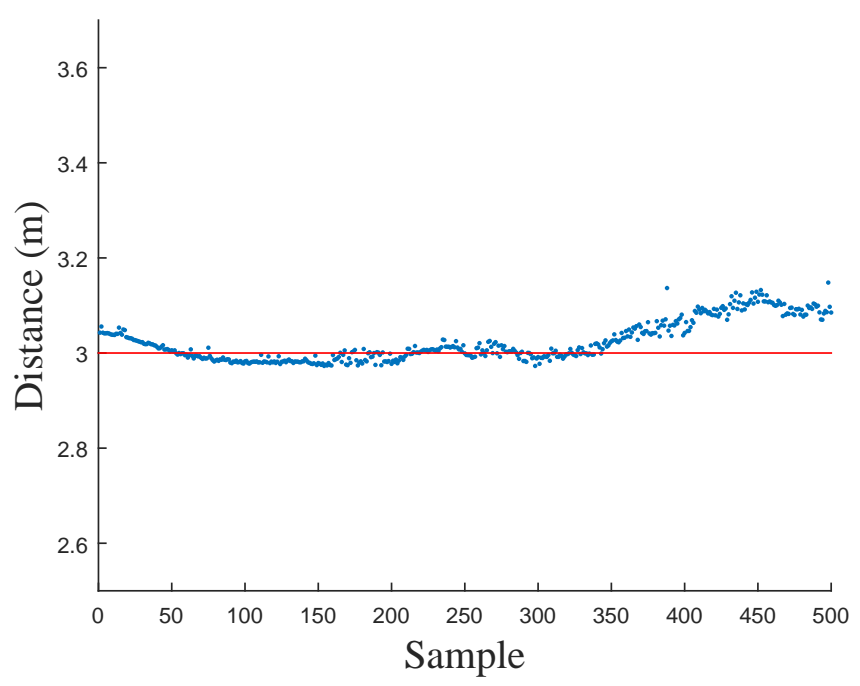

Figure A.5: Horizontal deviation at $3.0 \mathrm{~m}$ : Trial 2. 


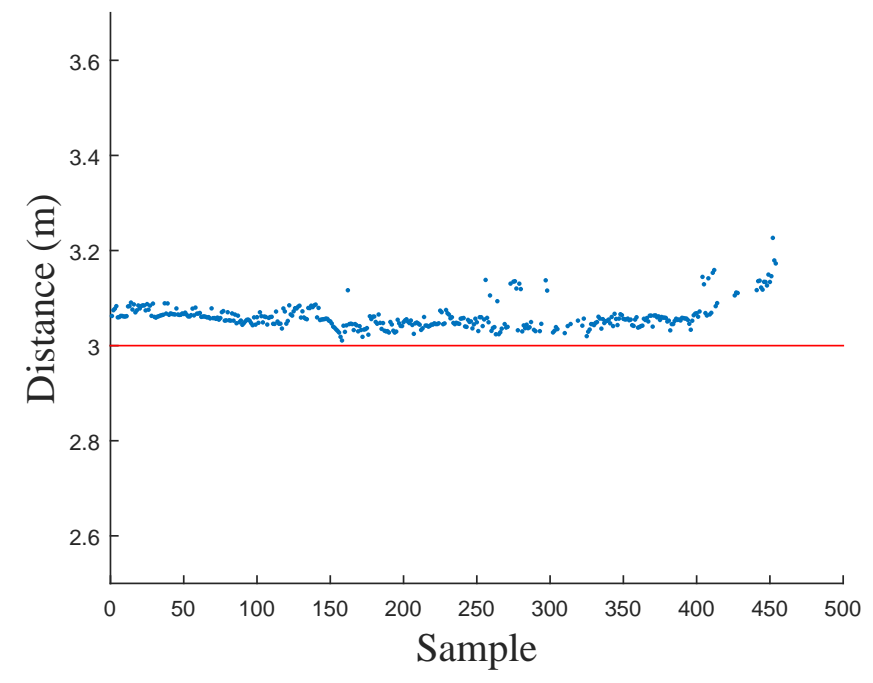

Figure A.6: Horizontal deviation at 3.0 m: Trial 3.

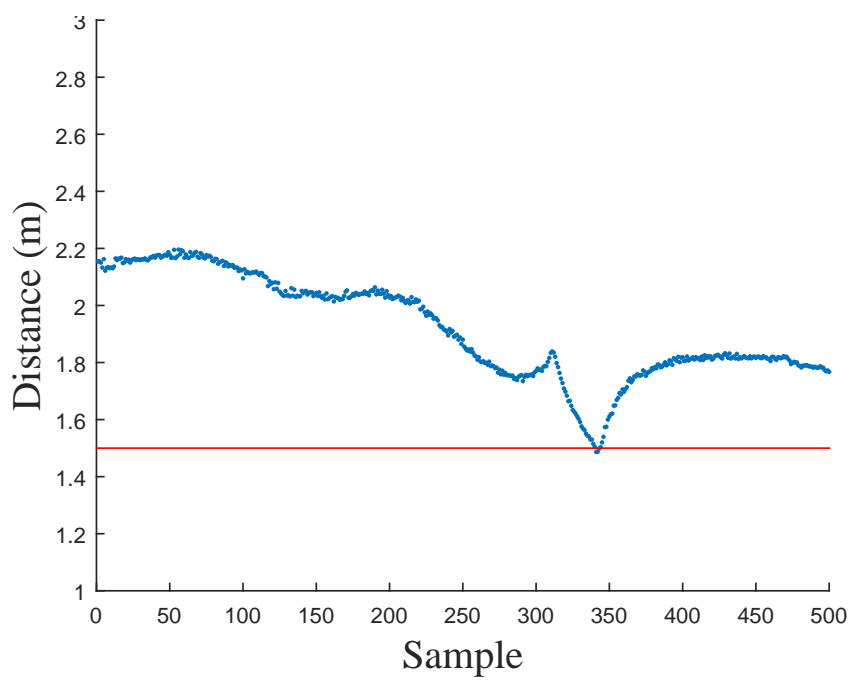

Figure A.7: Vertical deviation at $1.5 \mathrm{~m}$ : Trial 1. 


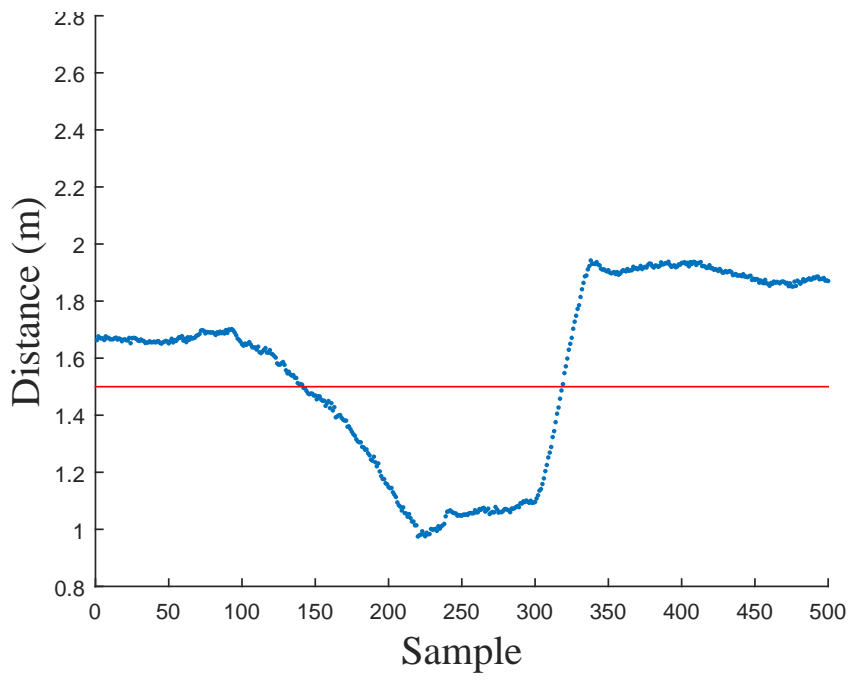

Figure A.8: Vertical deviation at $1.5 \mathrm{~m}$ : Trial 2.

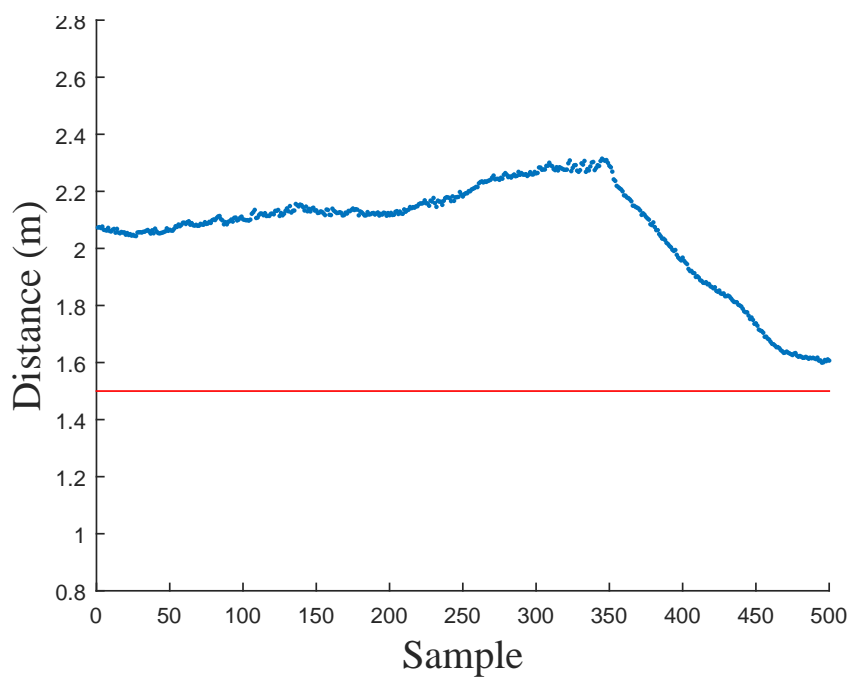

Figure A.9: Vertical deviation at $1.5 \mathrm{~m}$ : Trial 3. 


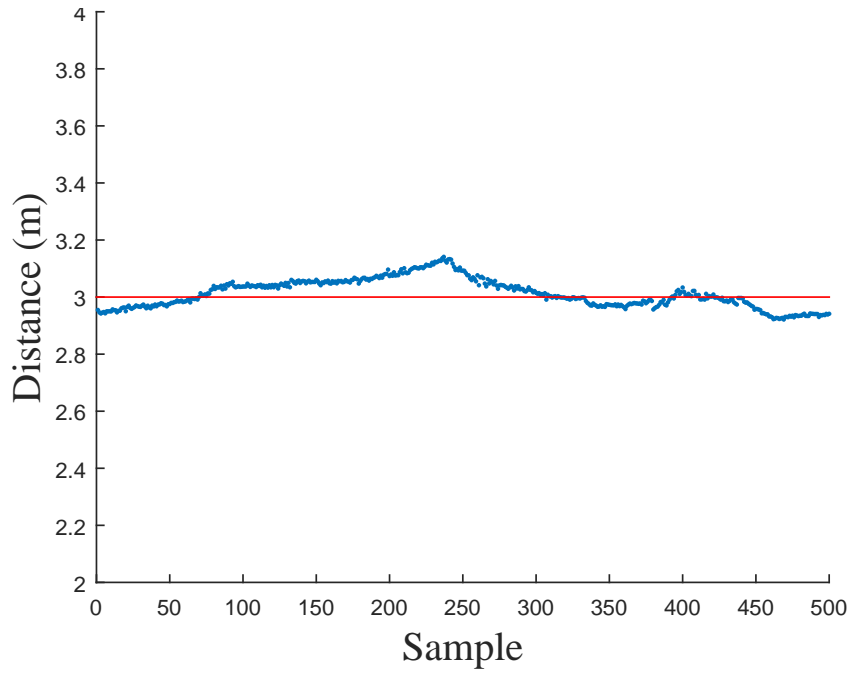

Figure A.10: Vertical deviation at $3.0 \mathrm{~m}$ : Trial 1.

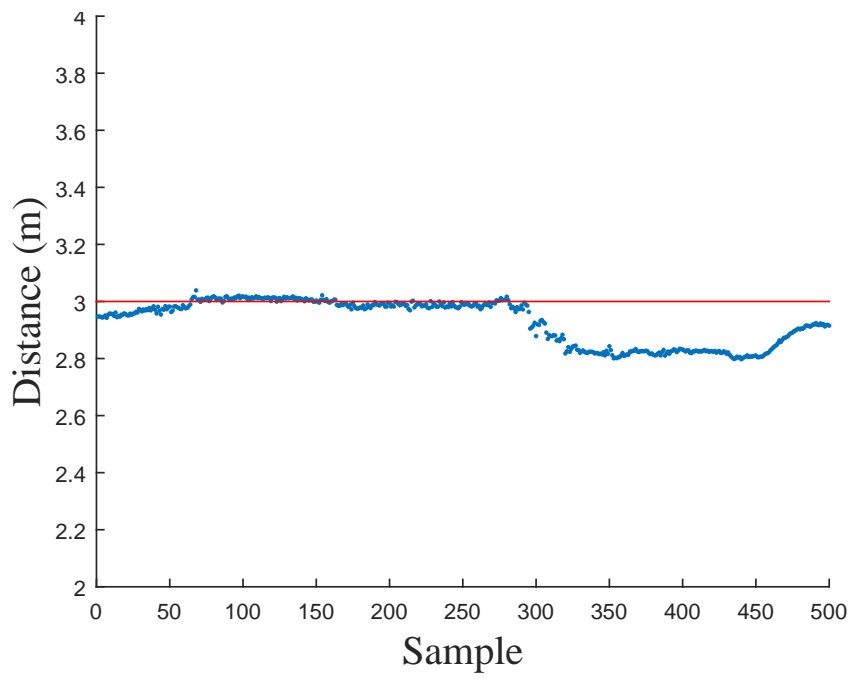

Figure A.11: Vertical deviation at $3.0 \mathrm{~m}$ : Trial 2. 


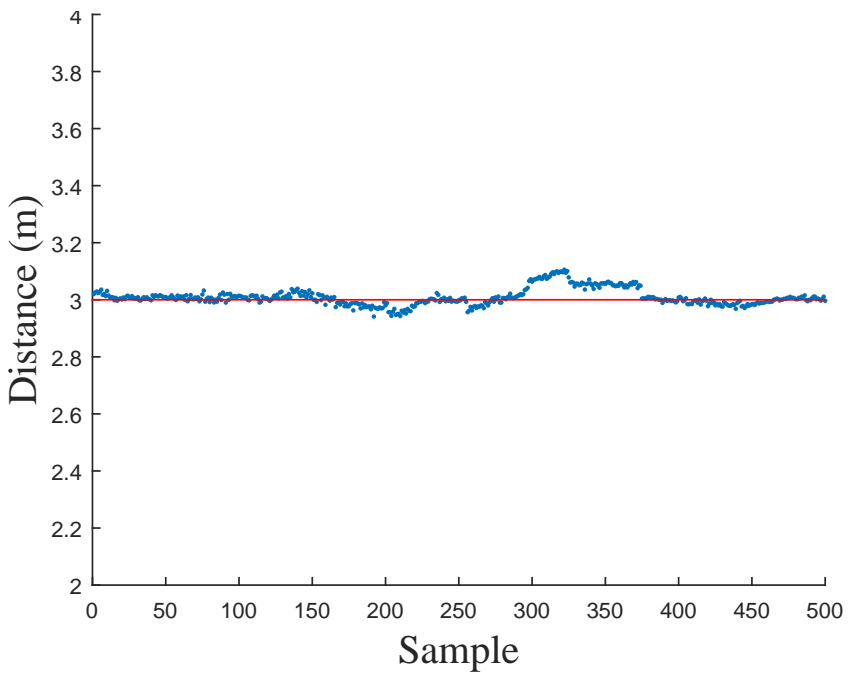

Figure A.12: Vertical deviation at $3.0 \mathrm{~m}$ : Trial 3. 


\section{APPENDIX B}

This appendix presents all flight midpoint pixel data for vertical and horizontal flight tests at distances of 1.5 and 3 meters. Data is adjusted in order to eliminate the occasional "jumps" and inconsistencies and converted from pixels to distance (m) using the baseline measurements from Table 7. Adjustments were made by either increasing or decreasing the data "jumps" along the pixel width axis so as to align it with the rest of the data. Data was converted from pixel width to distance in order to provide ease of visualization as to how far the aircraft was drifting from its intended target. Red lines indicate the average deviation distance from the $28 \mathrm{~cm}$ strip for the respective trial.

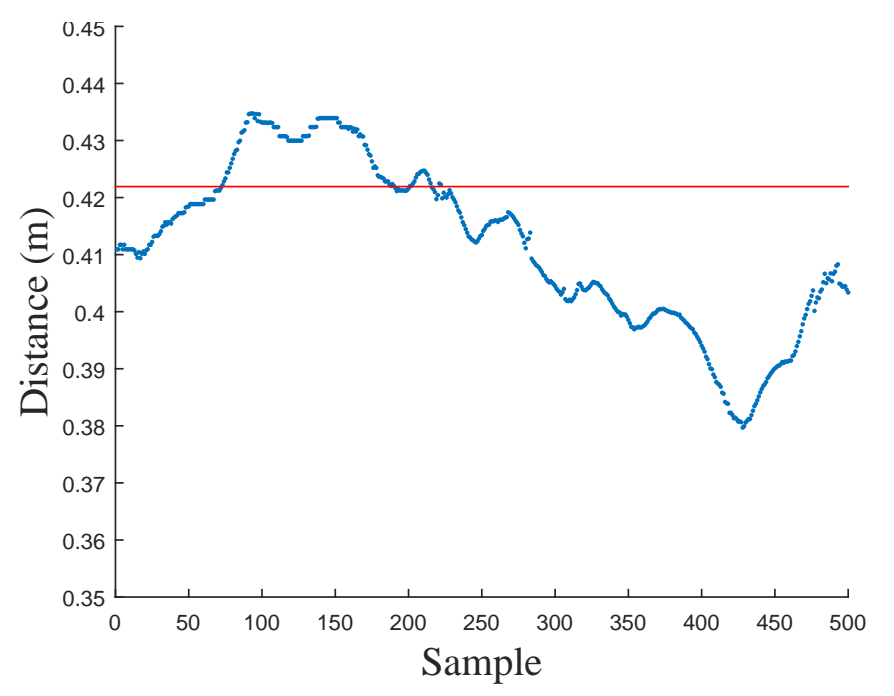

Figure B.1: Horizontal mid point deviation at $1.5 \mathrm{~m}$ : Trial 1. 


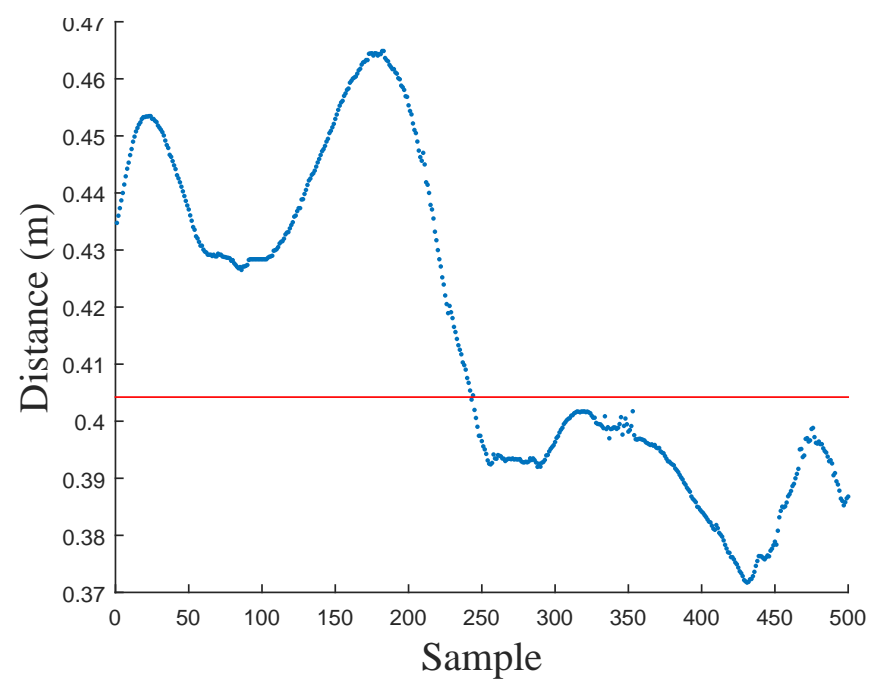

Figure B.2: VHorizontal mid point deviation at $1.5 \mathrm{~m}$ : Trial 2.

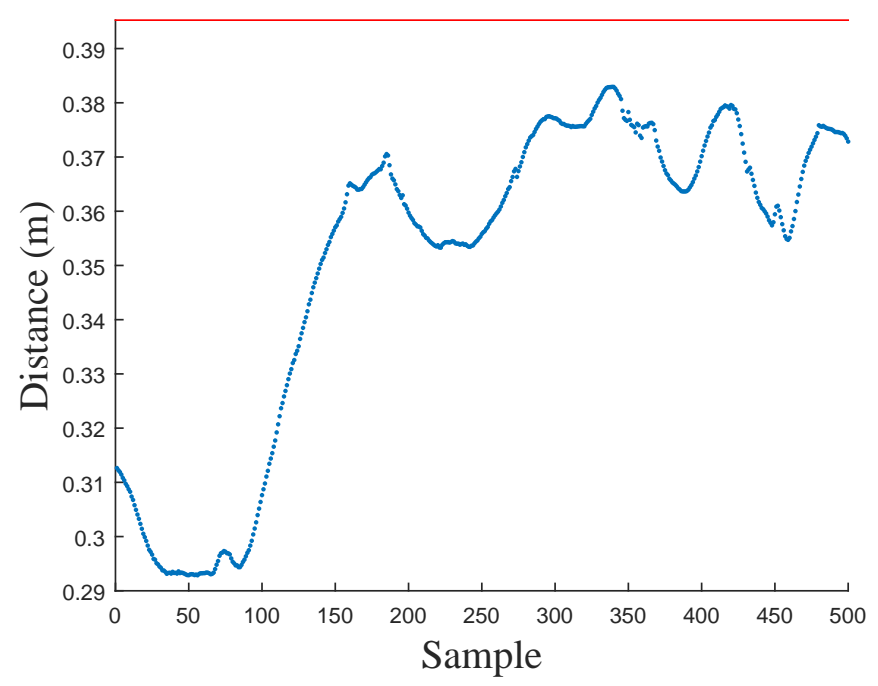

Figure B.3: Horizontal mid point deviation at $1.5 \mathrm{~m}$ : Trial 3. 


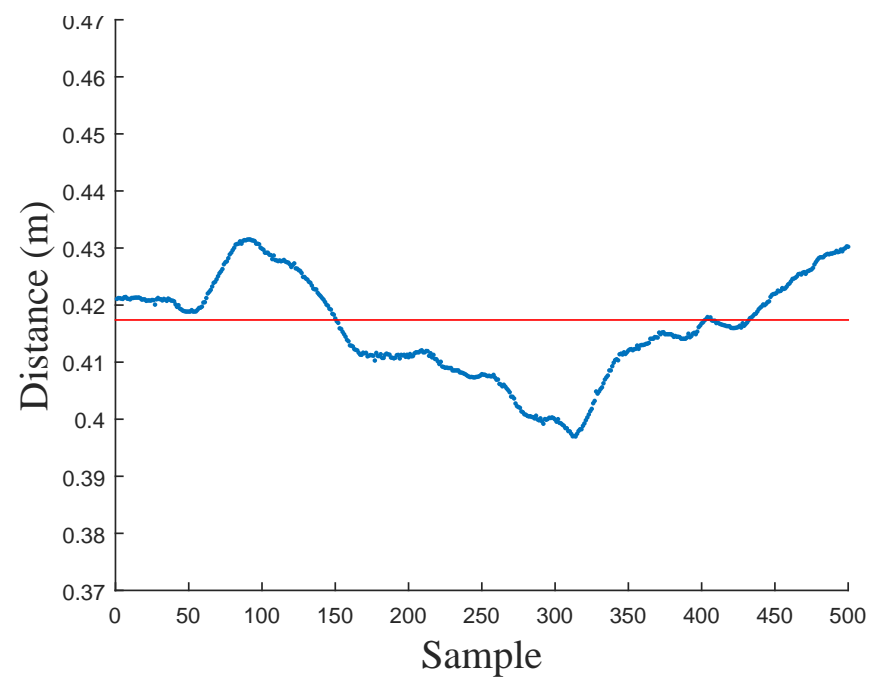

Figure B.4: Horizontal mid point deviation at $3.0 \mathrm{~m}$ : Trial 1.

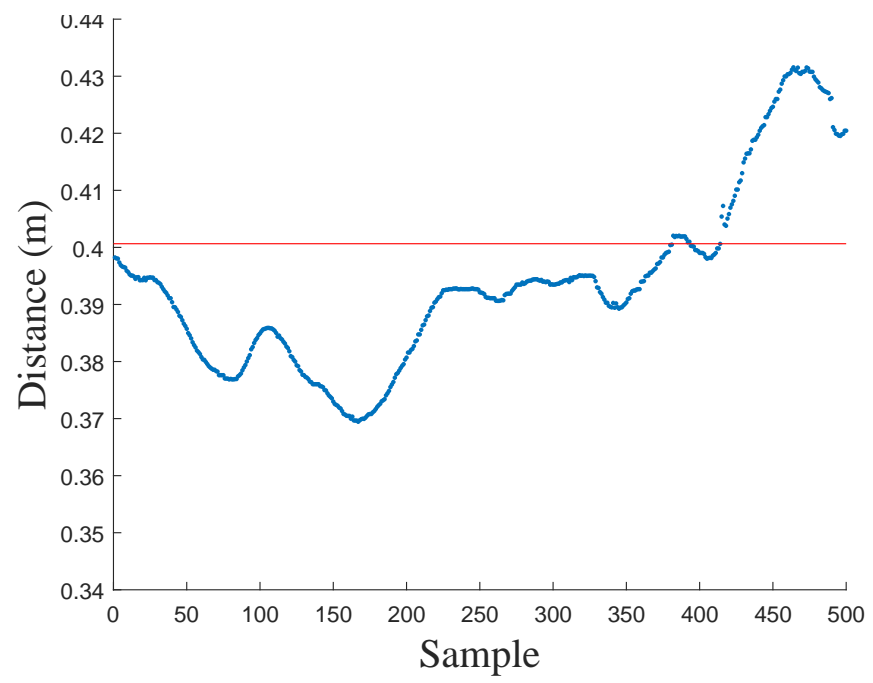

Figure B.5: Horizontal mid point deviation at $3.0 \mathrm{~m}$ : Trial 2. 


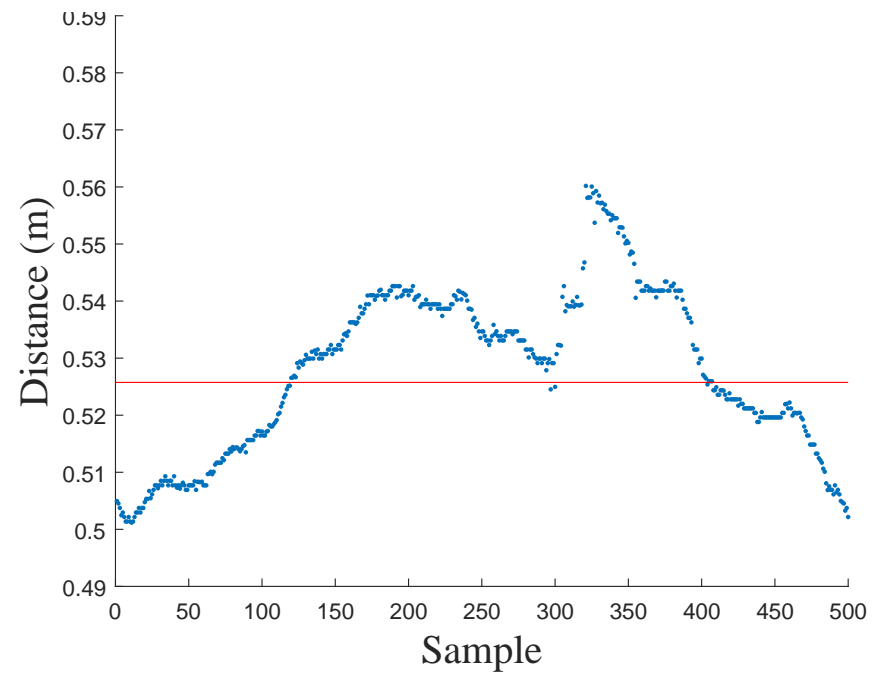

Figure B.6: Horizontal mid point deviation at $3.0 \mathrm{~m}$ : Trial 3.

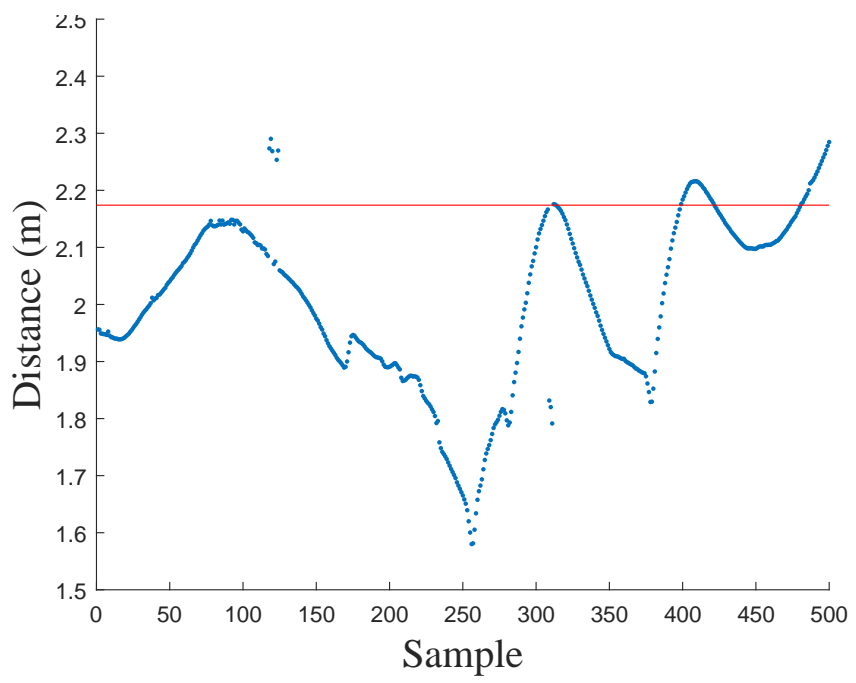

Figure B.7: Vertical mid point deviation at $1.5 \mathrm{~m}$ : Trial 1. 


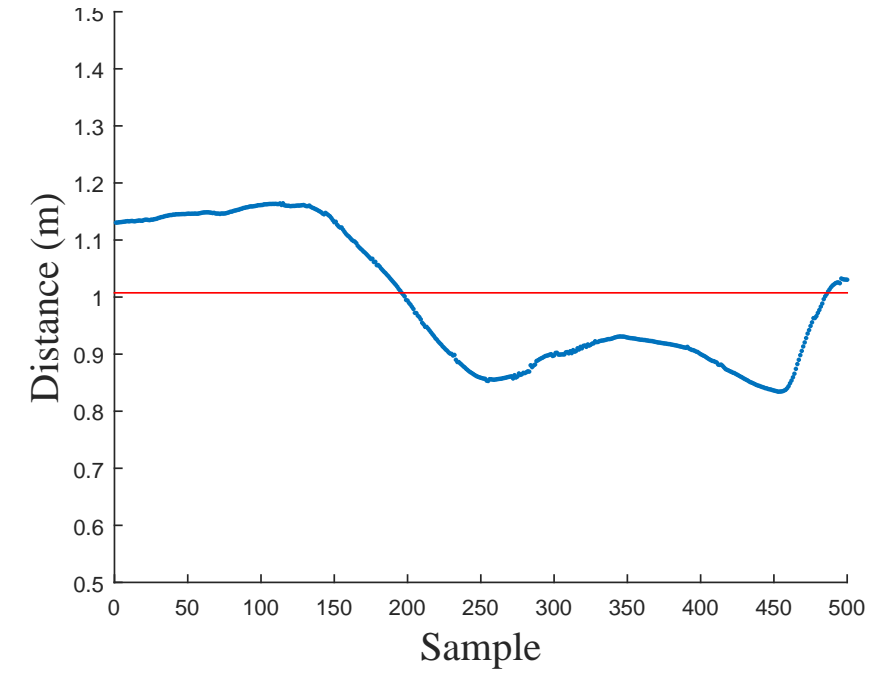

Figure B.8: Vertical mid point deviation at $1.5 \mathrm{~m}$ : Trial 2.

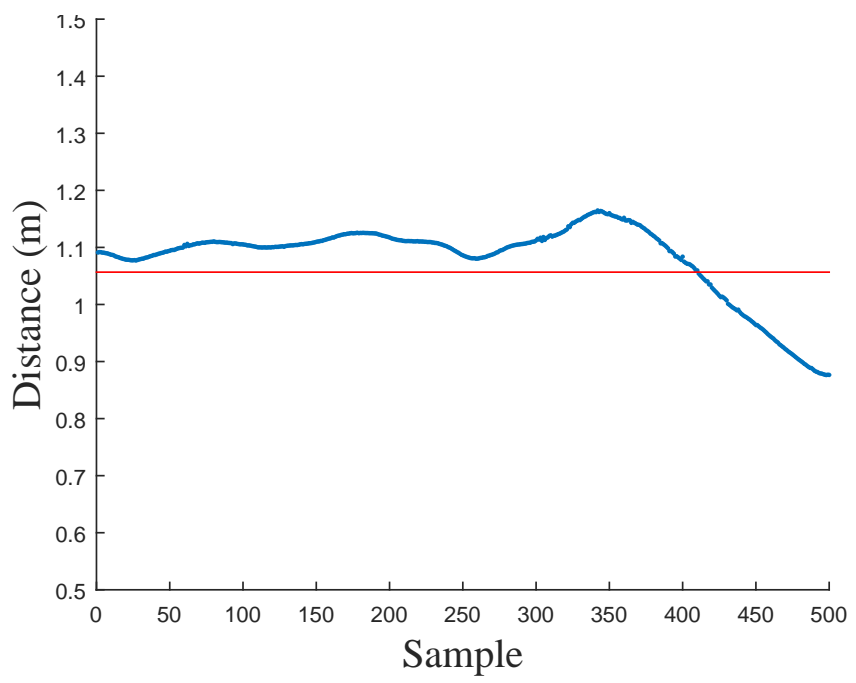

Figure B.9: Vertical mid point deviation at $1.5 \mathrm{~m}$ : Trial 3. 


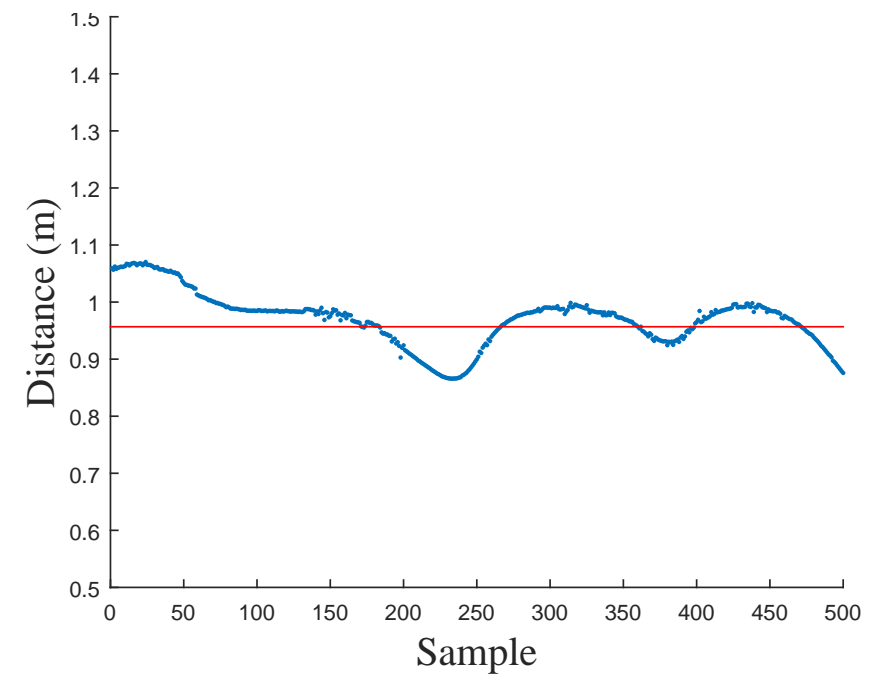

Figure B.10: Vertical mid point deviation at $3.0 \mathrm{~m}$ : Trial 1.

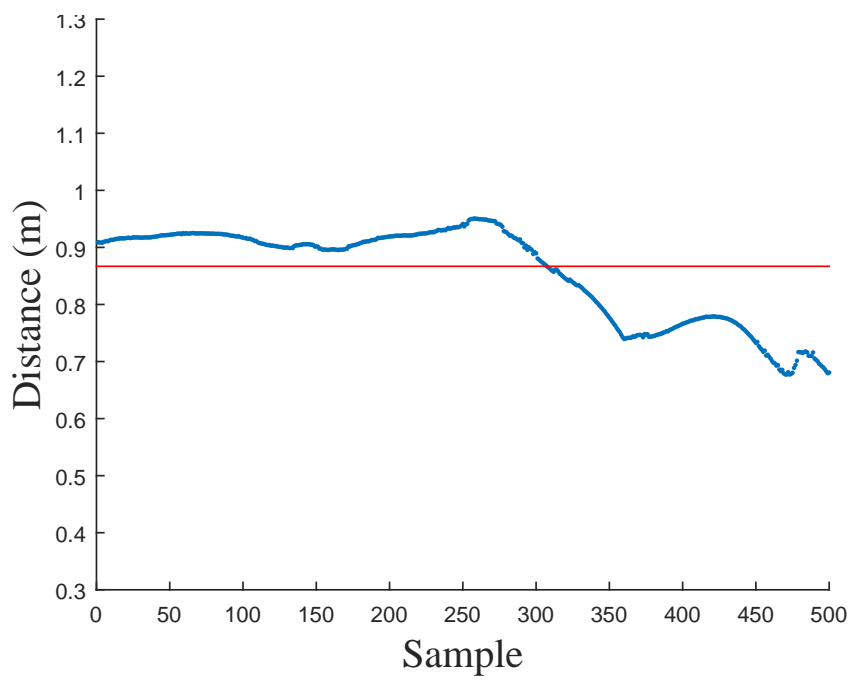

Figure B.11: Vertical mid point deviation at $3.0 \mathrm{~m}$ : Trial 2. 


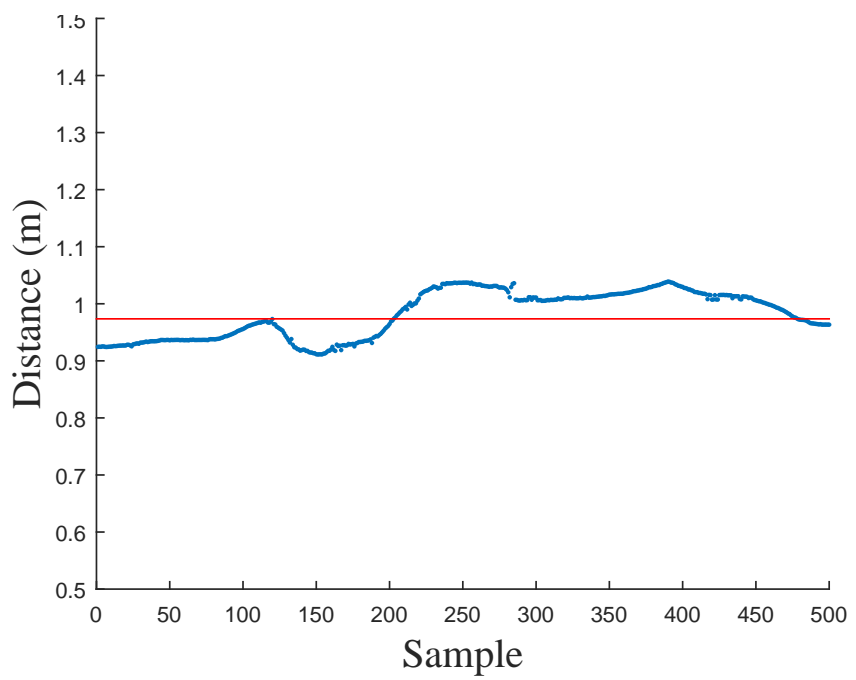

Figure B.12: Vertical mid point deviation at $3.0 \mathrm{~m}$ : Trial 3. 


\section{REFERENCE LIST}

[1] ASCE, 2013 Report Card for America's Infrastructure, American Society of Civil Engineers, 2013.

[2] Gao, J., Yan, Y., and Wang, C., "Research on the Application of UAV Remote Sensing in Geologic Hazards Investigation for Oil and Gas Pipelines," Proc., ICPTT 2011, 2011, pp. 381-390.

[3] Saleri, R., Pierrot-Deseilligny, M., Bardiere, E., Cappellini, V., Nony, N., De Luca, L., and Campi, M., "UAV Photogrammetry for Archaeological Survey: The Theaters Area of Pompeii," Digital Heritage International Congress (DigitalHeritage), 2013, Vol. 2, IEEE, 2013, pp. 497-502.

[4] Kaneko, K., Nohara, S., et al., "Review of Effective Vegetation Mapping Using the UAV (Unmanned Aerial Vehicle) Method," Journal of Geographic Information System, Vol. 6, No. 06, 2014, pp. 733.

[5] Gago, J., Douthe, C., Coopman, R., Gallego, P., Ribas-Carbo, M., Flexas, J., Escalona, J., and Medrano, H., "UAVs Challenge to Assess Water Stress for Sustainable Agriculture," Agricultural Water Management, Vol. 153, 2015, pp. 9-19.

[6] Mozas-Calvache, A., Pérez-García, J., Cardenal-Escarcena, F., Mata-Castro, E., and Delgado-García, J., "Method for Photogrammetric Surveying of Archaeological Sites with Light Aerial Platforms," Journal of Archaeological Science, Vol. 39, 
No. 2, 2012, pp. 521-530.

[7] Roca, D., Lagüela, S., Díaz-Vilariño, L., Armesto, J., and Arias, P., "Low-cost Aerial Unit for Outdoor Inspection of Building Façades," Automation in Construction, Vol. 36, 2013, pp. 128-135.

[8] Metni, N. and Hamel, T., “A UAV for BridgeIinspection: Visual Servoing Control Law with Orientation Limits," Automation in construction, Vol. 17, No. 1, 2007, pp. 3-10.

[9] Wang, K.-Y., Lee, P.-H., and Hung, S.-K., “Optimum Electric Cable Selection for Kite-like Unmanned Aerial Vehicle,” Advanced Intelligent Mechatronics (AIM), 2015 IEEE International Conference on, IEEE, 2015, pp. 1537-1540.

[10] Zikou, L., Papachristos, C., and Tzes, A., “The Power-over-Tether System for Powering Small UAVs: Tethering-line Tension Control Synthesis," Control and Automation (MED), 2015 23th Mediterranean Conference on, IEEE, 2015, pp. 681-687.

[11] Park, J., Im, S., Lee, K.-H., and Lee, J.-O., "Vision-based SLAM SYstem for Small UAVs in GPS-denied Environments," Journal of Aerospace Engineering, Vol. 25, No. 4, 2011, pp. 519-529.

[12] Mahmoud, K., Innovations in Bridge Engineering Technology: Selected Papers, 3rd NYC Bridge Conf., 27-28 August 2007, New York, USA, CRC Press, 2007.

[13] McDaid, H., Oliver, D., Strong, B., and Israel, K., "Remote Piloted Aerial Vehicles: An Anthology," 2003. 
[14] ScientificAmerican, “The Traveling Balloon,” Scientific American, Vol. 4, No. 24, mar 1849.

[15] Doherty, P. and Rudol, P., "A UAV Search and Rescue Scenario with Human Body Detection and Geolocalization,' AI 2007: Advances in Artificial Intelligence, Springer, 2007, pp. 1-13.

[16] Smith, M. J., Hamruni, A. M., and Jamieson, A., “3-D urban Modelling Using Airborne Oblique and Vertical Imagery,” ISPRS Hannover Workshop, 2009.

[17] Nikolic, J., Burri, M., Rehder, J., Leutenegger, S., Huerzeler, C., and Siegwart, R., “A UAV System for Inspection of Industrial Facilities," Aerospace Conference, 2013 IEEE, IEEE, 2013, pp. 1-8.

[18] Mejias, L., Correa, J. F., Mondragón, I., and Campoy, P., “COLIBRI: A Visionguided UAV for Surveillance and Visual Inspection,” 2007.

[19] Chen, J. Y., “UAV-guided Navigation for Ground Robot Tele-operation in a Military Reconnaissance Environment,” Ergonomics, Vol. 53, No. 8, 2010, pp. 940-950.

[20] Trammell III, H. S., Perry, A. R., Kumar, S., Czipott, P. V., Whitecotton, B. R., McManus, T. J., and Walsh, D. O., “Using Unmanned Aerial Vehicle-borne Magnetic Sensors to Detect and Locate Improvised Explosive Devices and Unexploded Ordnance," Defense and Security, International Society for Optics and Photonics, 2005, pp. 963-971. 
[21] Emelianov, S., Bulgakow, A., and Sayfeddine, D., "Aerial Laser Inspection of Buildings Facades Using Quadrotor," Procedia Engineering, Vol. 85, 2014, pp. 140-146.

[22] Zhang, J., Liu, L., Wang, B., Chen, X., Wang, Q., and Zheng, T., "High Speed Automatic Power Line Detection and Tracking for a UAV-based Inspection," Industrial Control and Electronics Engineering (ICICEE), 2012 International Conference on, IEEE, 2012, pp. 266-269.

[23] Li, Z., Liu, Y., Hayward, R., Zhang, J., and Cai, J., "Knowledge-based Power Line Detection for UAV Surveillance and Inspection Systems," Image and Vision Computing New Zealand, 2008. IVCNZ 2008. 23rd International Conference, IEEE, 2008, pp. 1-6.

[24] Deng, C., Wang, S., Huang, Z., Tan, Z., and Liu, J., "Unmanned Aerial Vehicles for Power Line Inspection: A Cooperative Way in Platforms and Communications," Journal of Communications, Vol. 9, No. 9, 2014.

[25] Jones, D., "Power Line Inspection: A UAV concept," Autonomous Systems, 2005. The IEE Forum on (Ref. No. 2005/11271), IET, 2005, pp. 8-pp.

[26] Fujii, K., Higuchi, K., and Rekimoto, J., "Endless Flyer: a Continuous Flying Drone with Automatic Battery Replacement," Ubiquitous Intelligence and Computing, 2013 IEEE 10th International Conference on and 10th International Conference on Autonomic and Trusted Computing (UIC/ATC), IEEE, 2013, pp. 216-223. 
[27] Vaghefi, K., Oats, R. C., Harris, D. K., Ahlborn, T. T. M., Brooks, C. N., Endsley, K. A., Roussi, C., Shuchman, R., Burns, J. W., and Dobson, R., "Evaluation of Commercially Available Remote Sensors for Highway Bridge Condition Assessment," Journal of Bridge Engineering, Vol. 17, No. 6, 2011, pp. 886-895.

[28] Chen, S.-E., Rice, C., Boyle, C., and Hauser, E., "Small-format Aerial Photography for Highway-bridge Monitoring," Journal of Performance of Constructed Facilities, Vol. 25, No. 2, 2011, pp. 105-112.

[29] Estes, A. C. and Frangopol, D. M., "Updating Bridge Reliability Based on Bridge Management Systems Visual Inspection Results," Journal of Bridge Engineering, Vol. 8, No. 6, 2003, pp. 374-382.

[30] Moller, P. S., “CALTRANS Bridge Inspection Aerial Robot Final Report,” Tech. rep., 2008.

[31] Lovelace, B., "Unmanned Aerial Vehicle Bridge Inspection Demonstration Project," Minnesota Department of Transportation, 2015.

[32] Karpowicz, R., "The Use of Unmanned Aerial Systems for Steep Terrain Investigations," Caltrans Division of Research, Innovation and System Information, 2014.

[33] Otero, L. D., Gagliardo, N., Dalli, D., Huang, W., and Cosentino, P., "Proof of Concept for Using Unmanned Aerial Vehicles for High Mast Pole and Bridge Inspections," Contract BDV28, 2015, pp. 977-02.

[34] Yin, Z., "Report\# MATC-MS\&T: 295 Final Report,” 2015. 
[35] Nicotra, M. M., Naldi, R., Garone, E., and Studiorum, A. M., “Taut Cable Control of a Tethered UAV," 19th IFAC World Congress, Cape Town, South Africa, 2014, pp. $3190-3195$.

[36] Muttin, F., “Umbilical Deployment Modeling for Tethered UAV Detecting Oil Pollution from Ship," Applied Ocean Research, Vol. 33, No. 4, 2011, pp. 332-343.

[37] Tognon, M., Dash, S. S., and Franchi, A., “Observer-based Control of Position and Tension for an Aerial Robot Tethered to a Moving Platform," 2016.

[38] Pratt, K. S., Murphy, R. R., Burke, J. L., Craighead, J., Griffin, C., and Stover, S., "Use of Tethered Small Unmanned Aerial System at Berkman Plaza II Collapse," Safety, Security and Rescue Robotics, 2008. SSRR 2008. IEEE International Workshop on, IEEE, 2008, pp. 134-139.

[39] Papachristos, C. and Tzes, A., “The Power-tethered UAV-UGV Team: A Collaborative Strategy for Navigation in Partially-mapped Environments," Control and Automation (MED), 2014 22nd Mediterranean Conference of, IEEE, 2014, pp. 11531158.

[40] Samarathunga, W., Wang, G., and Wang, S., "Vehicle Design of Tethered Hexaroters for Heavy Payload Applications," Computational Intelligence \& Communication Technology (CICT), 2015 IEEE International Conference on, IEEE, 2015, pp. 554556. 
[41] RCHeliNation, "How To: Setting Up the HP DPS-1200FB Power Supply - RC Heli Nation,” 2013 (accessed June 9 2014).

[42] Cooper, G. E. and Harper Jr, R. P., "The Use of Pilot Rating in the Evaluation of Aircraft Handling Qualities,” Tech. rep., DTIC Document, 1969.

[43] Cotting, M. C., "UAV Performance Rating Scale Based on the Cooper-Harper Piloted Rating Scale," 49th AIAA Aerospace Sciences Meeting including the New Horizons Forum and Aerospace Exposition, 2011, p. 923. 
Ignacio Hernandez was born on April 23, 1990 in Evanston, Illinois. He went to public school and graduated from Sumner Academy in Kansas City, Kansas, in 2008. He then attended and graduated from the University of Missouri-Kansas City with a Bachelor of Science in mechanical engineering and in physics in July 2015. He completed his undergraduate degree while also starting his master's degree program in mechanical engineering at the University of Missouri-Kansas City through their BS/MS five year program.

Since June 2014, Mr. Hernandez has been employed as a Graduate Research Assistant at the University of Missouri-Kansas City. Upon completion of his degree requirements, Mr. Hernandez plans to pursue his research interests through a doctoral program. 